\title{
NONCOMMUTATIVE BLACK HOLES, THE FINAL APPEAL TO QUANTUM GRAVITY: A REVIEW
}

\author{
PIERO NICOLINI \\ Department of Mathematics and Computer Science, Consortium of Magneto-Fluid-Dynamics, \\ University of Trieste and INFN, National Institute for Nuclear Physics, Via Valerio 12 \\ Trieste, I-34127, Italy \\ Department of Physics, California State University Fresno, 2345 E. San Ramon Ave., \\ Fresno, CA 93740-8031, United States \\ Piero.Nicolini@cmfd.univ.trieste.it \\ Received 11 July 2008 \\ Revised 24 November 2008
}

\begin{abstract}
We present the state of the art regarding the relation between the physics of Quantum Black Holes and Noncommutative Geometry. We start with a review of models proposed in the literature for describing deformations of General Relativity in the presence of noncommutativity, seen as an effective theory of Quantum Gravity. We study the resulting metrics, proposed to replace or at least to improve the conventional black hole solutions of Einstein's equation. In particular, we analyze noncommutative-inspired solutions obtained in terms of quasi-classical noncommutative coordinates: indeed because of their surprising new features, these solutions enable us to circumvent long standing problems with Quantum Field Theory in Curved Space and to cure the singular behavior of gravity at the centers of black holes. As a consequence, for the first time, we get a complete description of what we may call the black hole SCRAM, the shut down of the emission of thermal radiation from the black hole: in place of the conventional scenario of runaway evaporation in the Planck phase, we find a zero temperature final state, a stable black hole remnant, whose size and mass are determined uniquely in terms of the noncommutative parameter $\theta$. This result turns out to be of vital importance for the physics of the forthcoming experiments at the LHC, where mini black hole production is foreseen in extreme energy hadron collisions. Because of this, we devote the final part of this review to higher dimensional solutions and their phenomenological implications for TeV Gravity.
\end{abstract}

Keywords: Black Hole Evaporation; Noncommutative Gravity; Mini Black Hole.

PACS numbers: 04.70.Dy, 04.60.Bc, 11.10.Nx, 04.50.Gh

Per aspera ad astra Seneca, "Hercules furens" $2^{\text {nd }}$ Act, v. 437

\section{Introduction}

In 1975 Steven Hawking showed, in a remarkable paper ${ }^{1}$, that black holes can evaporate, namely that they can emit thermal radiation like a black body. This 
conjecture opened the window towards the mysteries of Quantum Gravity, since, at that time, the reconciliation between Quantum Mechanics and General Relativity was kept in a lethargic state as a matter of secondary concern. Indeed, theoretical physicists were basically interested in the Standard Model of particle physics and gravity was simply assumed to be too weak with respect to the already unified fundamental interactions. Even strings were employed as a theory of hadrons. Direct quantization of General Relativity into a quantum field theory in which the gravitational force is carried by gravitons led to the unpleasant appearance of infinities. This lack of renormalization was somehow an underestimated problem or at least considered as being too far away, only relevant at a scale 19 orders of magnitude higher than the mainstream high energy physics at the time. Therefore Gravity was kept classical and the major efforts were directed towards the Standard Model of particle physics, calculating Feynman diagrams, cross sections and decay rates. In this spirit, Hawking's conjecture about black hole evaporation was supported by semiclassical arguments 24 which hold only when the energy of the emitted particle is small compared to the black hole mass and it is possible to neglect the back reaction on the metric during the emission proces a. After realizing that black holes can emit photons, gravitons and other elementary particles as products of the decay, Hawking raised a natural and profound question about the final stage of the evaporation and the possible loss of information encoded in quantum states of matter. All of the troubles about the black hole evaporation were basically due to a breakdown of the semiclassical description, which requires that the black hole mass $M_{B H} \gg M_{P}$, where $M_{P}$ is Planck mass. As the decay proceeds and the black hole mass decreases, this condition eventually can no longer be met and a quantum theory of gravity must be invoked. Indeed the Planck scale marks the limes between the classical and the quantum behavior of the spacetime.

After more than 30 years of intensive research, the scenario is now drastically different and we have at least two plausible candidate theories of Quantum Gravity: Superstring: $\mathrm{b}$ and Loop Quantum Gravity 25 . Both of them have great merits and still-open problems, but at present both suffer from a basic limitation: the absence of any support from experiments. For this reason they cannot be considered as being more than theoretical speculations, even if they are both physically very promising, aesthetically very attractive and mathematically elegant and well defined. The main problem is that we do not yet have any experimental data demonstrating some quantum gravitational effect. From historical, epistemological and philosophical points of view, the long-standing inability to find observable effects of Quantum

\footnotetext{
${ }^{a}$ The physical picture of Hawking radiation is in terms of particles tunnelling through the horizon. Only recently has a transparent derivation of it been given in terms of a quasi-classical tunnelling calculation (see Refs. $5+22$ ).

${ }^{\mathrm{b}}$ In (Super)String Theory one adopts 1-dimensional quantum objects instead of the more conventional point-like structures 2324 . This represents the first chapter in a program for studying the physics of extended quantum objects, which also include 2-dimensional branes and 3-dimensional bubbles (for more details see Refs. 26-34).
} 
Gravity is probably the most dramatic crisis in theoretical physics and perhaps in all of science. What makes the situation even more embarrassing is that Quantum Gravity is expected to be the synthesis of two physical theories which are the most successful ever formulated from the point of view of their capacity for reproducing experimental result@.

What is the role of black holes in this context? It is very likely that black holes are fated to provide the final answer about our knowledge of Quantum Gravity and close the logical path which they started in 1975, igniting the initial interest in this area. Indeed, in the next few monthed we could be very close to having revolutionary experimental evidence for both particle phenomenology and gravitational physics: the detection of some signature of the production of mini black holes 38 - 40 that are predicted to be produced as a result of high energy hadronic collisions. According to theory, decay of a mini black hole would lead to the sudden emission of a huge quantity of particles independently of their spin, charge, quantum numbers or interaction properties, as long as their rest mass is smaller than the black hole temperature 41 . Therefore mini black holes may be excellent intermediate states for long-sought but yet undiscovered particles, like the Higgs boson or supersymmetric particles, possibly with cross sections much larger than for the direct processes.

For these reasons, the starting-up of the LHC, or of some other future experiment:E, will open up very good opportunities for getting information about Quantum Gravity $\underline{42}$ and the very early universe, as well as for solving some basic questions, whose answers are too often taken for granted.

First, do (mini) black holes exist? From velocity measurements for the whirlpool of hot gas surrounding it, astronomers have found convincing evidence for the existence of a supermassive black hole in the center of the giant elliptical galaxy M87f. Astronomers have also detected radio emission coming from within 30 million kilometers of the dark object SGR A*, thought to be a colossal black hole, that lies at the center of the Milky Way 53 . Previously, X-ray emission from the binary star system Cygnus X-1 convinced many astronomers that it contains a black hole, which is supported by more precise measurements which have recently become available. To this purpose we also have to mention V404 Cygni, one of the most evident cases

\footnotetext{
${ }^{\mathrm{c}}$ The relative error in the General Relativity prediction for the rate of change of the orbital period of the Hulse-Taylor binary pulsar PSR $1913+16$ is $10^{-14}$ (see Ref. [35). Experimental data and Quantum Field Theory predictions of the value of the anomalous magnetic moment of the electron agree to within a relative error of $10^{-11}$ (see Ref. 36).

d The Large Hadron Collider (LHC) is being built at the CERN lab and is planned to circulate the first beams in August 2008, while the first collisions are expected in October 2008 with the first results arriving soon afterwards 37 .

${ }^{\mathrm{e}}$ Formation and detection of mini black holes is expected in both future hadron colliders, such as the Very Large Hadron Collider (VLHC) at Fermilab, and the particle shower of Ultra High Energy Cosmic Rays (UHECR), impacting Earth's atmosphere 42 .

${ }^{\mathrm{f}}$ In 1994, Hubble Space Telescope data produced an unprecedented measurement of the mass of an unseen object at the center of M87. Based on the kinetic energy of the material whirling about the center, the object is estimated to be about 3 billion times the mass of our Sun and appears to be concentrated within a volume of space smaller than that of our solar system.
} 
of stellar black hole: observations at X-ray and optical wavelengths have shown that this is a binary system in which a late yellowish $\mathrm{G}$ star or maybe an early orangered $\mathrm{K}$ star revolves, every 6.47 days, around a compact companion with a diameter around $60-90 \mathrm{~km}$ and a probable mass of 8 to 15 solar masses, well above the mass limit at which a collapsed star must become a black hol 5762 . In spite of these observations, however, there are still some ranges of mass in which the existence of black holes is unclear, in particular of black holes of less than 3 solar masses. The relevance of these objects is connected with the possibility of observing the Hawking radiation. Indeed, what we know for sure is that for astrophysical black holes the Hawking radiation is negligible because their temperatures can be at most some tens of $\mathrm{nK}$, far below $T_{\mathrm{CMB}} \sim 2.7 \mathrm{~K}$, the temperature of Cosmic Microwave Background $(\mathrm{CMB})$ radiation. On the other hand tidal effects are significant in the case of mini black holes that could be very hot and very bright if their mass is sufficiently small 63 .

As second point, we might be able to conclude that (mini) black holes really can evaporate. Indeed for the above reasons the detection of these objects is the unique direct wayg to have experimental evidence of the Hawking conjecture, one of the most important predictions of Quantum Field Theory in Curved Spacetime and of the associated semiclassical gravity.

Thirdly, we could find out what it is the fate of a radiating black hole. If mini black holes can be created in high energy particle collisions, the black holes produced will pass through a number of phases before completing their evaporation. After a loss during the first two phases of their "hair" (i.e. the associated long-range fields) and of their angular momentum (the "balding" and spin-down phases respectively), the picture of the evaporation will be described by the Schwarzschild phase, in which the resulting spherically symmetric black hole loses energy by the emission of Hawking radiation, with a gradual decrease in its mass and an increase in its temperature. Since the Schwarzschild geometry has a curvature singularity at the origin, there would be a divergent behavior of the Hawking temperature if the black hole were to shrink to the origin as a result of losing mass by thermal emission. However, we do not expect that this divergent behavior will, in fact, take place since in the vicinity of the origin, the evaporating black hole will be dramatically disturbed by strong quantum gravitational fluctuations of the spacetime manifold. In other words the black hole will undergo a Plank phase of the evaporation during

g There exist alternative proposals to observe the Hawking radiation by an "indirect" means in the so-called Analogue Models, namely condensed matter systems which behave, to some extent, like gravitational ones 64: in Bose-Einstein condensates the supersonic region is the acoustic analog of a black hole, while the flux of phonons corresponds to the Hawking radiation. The resulting Hawking temperature is $T_{H} \sim 10 \mathrm{nK}$ as compared with a condensate temperature $T_{c} \sim 100 \mathrm{nK}$ (see Ref. 65 for a review and Refs. 66 67 for recent results).

Another interesting proposal is that concerning the possibility of experimentally detecting the Unruh effect in particle storage rings in terms of the Sokolov-Ternov effect $68 \mid 69$. This could be the simplest prototype of vacuum polarization effects in curved space. 
which a theory of Quantum Gravity must be used. Observations of the final stages of black hole evaporation could provide the profile of the temperature as a function of the mass of the black hole and hence let us pick out, for the first time, the correct quantum gravitational theory.

All of this promising and very fascinating program is, on the other hand, subject to a severe and unavoidable constraint: the inferred existence of large spatial extra dimensions. This hypothesis is currently considered to be the unique viable solution to the long-standing hierarchy problem, namely the presence of two fundamental scales in nature, the electroweak scale and the Planck scale, separated by 16 orders of magnitude 70 . The main point in this potential resolution of the problem is that extra dimensions can be assumed to be as large as around a millimeter, if we suppose that Standard Model fields are constrained in a four dimensional sub-manifold of the higher dimensional spacetime, and that only gravity can probe the additional transverse dimensions. In connection with this, recent experiments involving direct measurement of Newtonian gravity put limits on the size of extra dimensions at length scales below $1 \mathrm{~mm} \cdot{ }^{80181} \mathrm{In}$ this type of scenario, we can identify a new scale $M_{*}$, derived from the Planck scale through the following relation

$$
M_{*}^{(2+n)}=M_{P}^{2} / R^{n}
$$

where $R$ is the mean size of each of the $n$ extra dimensions. If $R$ is large enough with respect the Planck length $\ell_{P}$, then $M_{*}$ will be much smaller than $M_{P}$ and we obtain a unique fundamental scale $M_{*} \sim 1 \mathrm{TeV}$ for both the electroweak and gravitational interactions. Conversely, in the absence of any large extra dimensions, Quantum Gravity is lost forever: it is very likely that at the current rate of technological progress, mankind will probably become extinct before any experimental evidence of Quantum Gravity would become accessible. Giving a possible solution of the hierarchy problem is not the only key consequence of the presence of large extra dimensions: there are also further important consequences which we will summarize briefly here. First, any black hole smaller than the size of the extra dimensions can be considered, to a good approximation, as being totally submerged in a $4+n$ dimensional isotropic spacetime, with one time dimension and $3+n$ spatial ones. This allows one to use the higher-dimensional Schwarzschild solution to describe at least the neutral non-rotating phase of the black hole's life. As a result, the cross section for creation of mini black holes due to parton collisions significantly increases in the presence of large extra dimensions. Indeed, the corresponding Schwarzschild radii $r_{H}$ become of the order of $10^{-4} \mathrm{fm}$, which is large with respect to the parton impact parameter $b$. Therefore we can estimate the black hole production cross section by the geometrical approximation $\sigma \sim \pi r_{H}^{2} \sim 400 \mathrm{pb}$ and so, at the estimated luminosity for the LHC $\left(L \sim 10^{33} \mathrm{~cm}^{-2} \mathrm{~s}^{-1}\right)$ we find that roughly ten black holes would be created per second 82 . Another consequence of the introduction of large extra dimensions is that the mini black holes would be colder and thus live longer than their four-dimensional analogs, and so would be more easily detectable once created. Indeed, one typically finds mini black hole temperatures of the order of $100 \mathrm{GeV}$ 
and lifetimes of the order of $10^{-26} \mathrm{~s}$, that are interpreted as those of resonances.

At this point, the game seems to be over: we have candidate theories of Quantum Gravity and forthcoming experiments which are potentially able to check them. We should then try to find some easily testable theoretical predictions and wait for their experimental confirmation. The problem is that the final state of black hole evaporation cannot be efficiently described by means of the aforementioned theories of Quantum Gravity. Indeed (Super)String Theory provides a quantum description of black holes only for a few cases, namely for the extremal (and near-extremal) charged black hole models $83 \mid 84$. This is rather unsatisfactory, since the Planck phase, during the terminal stage of the evaporation, occurs in a neutral regime due to the rapid discharge of the black hole in the very initial stages. On the other hand, Loop Quantum Gravity suffers from the absence of a clear semiclassical limit. What is missing is a systematic way of computing scattering amplitudes and cross sections by perturbative techniques, a fact that is the basic limitation for obtaining significant quantitative results 25 . Therefore, in the absence of a full quantum description of all of the significant black hole evaporation phases, one uses effective theories to describe the quantum gravitational behavior, at least in some regimes. The most common effective tool is Quantum Field Theory in Curved Space, which works efficiently at least until the black hole quantum back reaction destroys the hypothesis of a fixed background spacetime 85 . Very recently, stimulated by the need for going beyond the rough semiclassical approximation of Quantum Field Theory in Curved Space, significant new approaches have been proposed based on Noncommutative Geometry arguments. Indeed there is a long-held belief that Quantum Gravity should have an uncertainty principle which prevents one from measuring positions to accuracies better than that given by the Planck length: the momentum and energy required to make such a measurement would themselves modify the geometry at these scales 87 . Therefore, one might wish to describe these effects, at least effectively, by a model theory having a new sort of uncertainty principle among the coordinates. In the same way as happens with coordinates and momenta in conventional quantum theory, the uncertainty would come from a noncommutative relation and so one is led to examine the possibility that position measurements might fail to commute, postulating the existence of a noncommutative manifold

$$
\left[\mathbf{x}^{i}, \mathbf{x}^{j}\right] \neq 0 \text {. }
$$

A feature of a Noncommutative Geometry would be the presence of quantum fluctuations able to remove the infinities which usually appear and cause the bad shortdistance behavior of field theories, including gravity.

The purpose of this review is to explore the current status of the physics of Quantum Black Holes from the viewpoint of Noncommutative Geometry, used as an effective tool for modelling the extreme energy quantum gravitational effects of the final phase of the evaporation, which are plagued by singularities at a semiclassical level. In Section 2, we start by reviewing the most popular Noncommutative Geometry models existing in the literature and their role in comparison with 
General Relativity. In Section 3, we discuss the four dimensional noncommutative Schwarzschild solution, from both geometrical and thermodynamical points of view. In Section 4, we study how noncommutativity affects the Maxwell field and present the four dimensional noncommutative Reissner-Nordström solution, providing a detailed analysis of both the Hawking and Schwinger pair production mechanisms. In Section 5, we consider evaporation in the extra-dimensional scenario, for both neutral and charged solutions, reviewing its phenomenological consequences in view of a possible experimental detection at the LHC. The final Section is devoted to future perspectives.

\section{Models of Fuzzy Geometry}

Noncommutativity is an old idea in theoretical physics, dating back to the early times of quantum theory and originally postulated as a way to improve the renormalizability properties of a theory at short distances or even to make it finite 92 . 97 . On the other hand, the development of efficient renormalizion techniques in field theories, decreased the interest in noncommutativity until recent times when the quantization of a unrenormalizable theory like gravity was studied extensively. In the 1980s, noncommutativity was largely developed by mathematicians in order to characterize a Euclidean manifold in terms of the spectrum of the Dirac operator on the manifold, providing a generalization of differential geometry, which became known as Noncommutative Geometry $98-106$.

In the high energy physics community, the interest about noncommutativity was revitalized when, in the Theory of Open Strings, it has been shown that target spacetime coordinates become noncommuting operators on a $D$-brane in the presence of a constant Neveu-Schwarz B field 107/108. As a result, the open strings, at least in the low energy limit when matter decouples from gravity, induced a quantum field theory on noncommutative spacetime, often called Noncommutative Field Theory and characterized by the transparent presence of a noncommutative coordinate algebra. In some sense, this physically motivated approach is somehow against the spirit of the mathematicians' community approach 98 - 106, which was formulated in a coordinate free language in terms of diffeomorphism invariants. Also in the framework of Loop Quantum Gravity, the resulting quantum geometry exhibits a noncommutative behavior: indeed operators that measure areas and volumes of regions of a spatial sub-manifold, strictly speaking fail to commute 109 -112.

Independently of its interpretation, namely as a fundamental picture of quantum spacetime or as a formalism capturing some aspects of String Theory and/or Loop Quantum Gravity, Noncommutative Geometry is currently employed to implement the "fuzziness" of spacetime by means of

$$
\left[\mathbf{x}^{i}, \mathbf{x}^{j}\right]=i \theta^{i j}
$$

where, in the simplest case, $\theta^{i j}$ is an anti-symmetric, real, $D \times D(D$ is the dimension of spacetime) matrix, which has dimension of a length squared. Such matrix 
determines the fundamental cell discretization of spacetime much in the same way as the Planck constant $\hbar$ discretizes the phase space. As a consequence, the resulting geometry is "pointless", since the notion of point is no longer meaningful because of the uncertainty

$$
\Delta x^{i} \Delta x^{j} \geq \frac{1}{2}\left|\theta^{i j}\right|
$$

induced by the noncommutative behavior of coordinates. Therefore one could interpret this loss of resolution as the emergence of a natural effective ultraviolet cut off, regulating not only gravity but any quantum field theory. If we define $\theta$ to be the average magnitude of an element of $\theta^{i j}$, physically $\frac{1}{\sqrt{\theta}}$ corresponds to the energy scale beyond which the conventional differential spacetime manifold turns out to be a noncommutative one. To this purpose, there is a general consensus about the appearance of noncommutative phenomenology at intermediate energies between the scale of the Standard Model of particle physics and the Planck scale. For this reason, the inclusion of noncommutativity in field theory in flat space can provide lower bounds about the energy threshold beyond which a particle moves and interacts in a distorted spacetime. The presence of noncommutative effects in flat space is, on the other hand, a feature which merits further inspection: the defining relation (3) provides a subtle process of quantization of the spacetime manifold $\mathcal{M}$, we define in terms of a class of equivalent atlases, without regard of any sort of field, function or tensor defined over it. Only after having constructed this groundwork we can think to introduce various kinds of structure over the manifold, even when $\mathcal{M}$ is subject to a noncommutative behavior. Among the possible structures, we can independently consider matter fields $\psi_{\alpha}$ propagating over $\mathcal{M}$ and any kind of tensorial quantity, including a symmetric $(0,2)$ non degenerate tensor, called metric tensor $g_{\mu \nu}$ describing the curvature properties of $\mathcal{M}$. For this reason, the quantization of the geometry encoded in (3) can be considered a genuine background independent procedure: indeed the metric tensor works as a mathematical apparatus superimposed on $\mathcal{M}$ and measures the gravitational interaction on the underlying spacetime manifold, much in the same way as the Newtonian gravitational field $\phi$ in the pre-relativistic physics. As a result, the quantum mechanical fluctuations of $g_{\mu \nu}$ are the response of the corresponding quantum fluctuations of $\mathcal{M}$ governed by the noncommutative relation (3). Therefore, the quantum behavior of $\mathcal{M}$ is an independent fact of metric tensor and can be considered even when only matter fields $\psi_{\alpha}$ are allowed to propagate on $\mathcal{M}$. The manifold $\mathcal{M}$, its fluctuations and curvature look similar to the case of a surface, that at short distances (high energies) appears rough, even if nothing is said whether the surface is still flat or already curved.

Noncommutativity could be also able to describe a possible Lorentz violation: the granular structure of space would mean that different wave lengths of light could travel at different speeds and play a role in the unexpected energy threshold of cosmic rays. However, the violation of Lorentz invariance may or may not occur in Noncommutative Geometry: indeed there exist many formulations, based on dif- 
ferent ways of implementing non-local deformations in field theories $\mathrm{h}$, starting from a noncommutative relation and preserving Lorentz symmetry

$$
\left[\mathbf{x}^{\mu}, \mathbf{x}^{\nu}\right]=i \theta^{\mu \nu}
$$

where now $\theta^{\mu \nu}$ is an anti-symmetric second-rank tensor 115 -121.

The most popular approach to Noncommutative Geometry is founded on the replacement of the point-wise multiplication of fields in the Lagrangian by a nonlocal Groenewold-Moyal $\star$-product 122123 . This technique, largely inspired by the foundations of quantum mechanics $124[125$, provides a systematic way to describe noncommutative spaces and to study field theories propagating thereon. Since physically, it turns out that $\theta$ is a free parameter, which we can imagine increasing from zero to go from the commutative to the noncommutative regime, a deformation of $C(\mathcal{M})$, the bounded continuous function on $\mathcal{M}$, is an algebra $\mathcal{A}_{\theta}$ with the same elements and addition law but different multiplication law, called $\star$-product to distinguish it from the original point-wise multiplication of functions. The notation has a further virtue, since let us somehow circumvent the ordering conditions in $\mathcal{A}_{\theta}$. Indeed, if we want to specify monomials or any kind of product of noncommutative coordinates, we have to give a ordering prescription. On the other hand, we can establish an isomorphism between the noncommutative algebra $\mathcal{A}_{\theta}$ and the conventional algebra of functions $C\left(\Re^{D}\right)$, employing a linear map $S$, called symbol of the operator. Therefore we represent the multiplication law in $\mathcal{A}_{\theta}$ in term of the

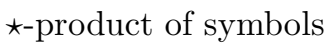

$$
\hat{f} \hat{g} \equiv S^{-1}[S[\hat{f}] \star S[\hat{g}]]
$$

where $\hat{f}, \hat{g} \in \mathcal{A}_{\theta}$. There could be many valid definitions of $S$, corresponding to different choices of ordering prescription for $S^{-1}$. A convenient and standard choice for $S^{-1}$ is the Weyl ordered symbol $\mathcal{W}$, largely used in the early times of quantum mechanics $124 \mid 125$. As a result we map the basis monomials in $C(\mathcal{M})$ onto the symmetrically ordered basis elements of $\mathcal{A}_{\theta}$, namely $x^{\alpha} x^{\beta} \rightarrow \frac{1}{2}\left(\mathbf{x}^{\alpha} \mathbf{x}^{\beta}+\mathbf{x}^{\beta} \mathbf{x}^{\alpha}\right)$. The extension of $\mathcal{W}$ to the algebra isomorphism, induced the Groenewold-Moyal $\star$-product $\mathcal{W}[f \star g] \equiv \mathcal{W}[f] \mathcal{W}[g]=\hat{f} \hat{g}$, where $f, g \in C(\mathcal{M})$ and $\hat{f}, \hat{g} \in \mathcal{A}_{\theta}$. The representation turns out to be

$$
f(x) \star g(x)=\sum_{k=0}^{\infty} \frac{1}{k !}\left(\frac{i}{2}\right)^{k} \theta^{\alpha_{1} \beta_{1}} \cdots \theta^{\alpha_{k} \beta_{k}} \partial_{\alpha_{1}} \cdots \partial_{\alpha_{k}} f(x) \partial_{\beta_{1}} \cdots \partial_{\beta_{k}} g(x)
$$

and the $\star$-product can be shown to be associative and noncommutative. Therefore, the spacetime noncommutativity may be encoded through ordinary products in the noncommutative $\mathcal{A}_{\theta}$ of Weyl operators, or equivalently through the deformation of the product of the commutative algebra of functions on spacetime $C(\mathcal{M})$ to the noncommutative $\star$-product.

${ }^{\mathrm{h}}$ For a review about this topic see Refs. 113114 
To transform a conventional field theory into a noncommutative one, we may write the field action in terms of Weyl operator $\mathcal{W}[\phi]$, corresponding to the field $\phi(x)$; then we can map the action back to coordinate space to get

$$
S=\int d^{D} x\left[\frac{1}{2}\left(\partial_{\mu} \phi\right) \star\left(\partial^{\mu} \phi\right)-\frac{m^{2}}{2}(\phi \star \phi)+\frac{\lambda}{24}(\phi \star \phi \star \phi \star \phi)\right] .
$$

In spite of its mathematical exactitude, the $\star$-deformed field theory suffers nontrivial limitations. The Feynman rules, obtained directly from the above action, lead to unchanged propagators, which implies that the $\star$-deformed field theory is identical to the ordinary field theory at the level of free fields, a fact somehow physically counterintuitive or at least unexpected. On the other hand, the only modifications, concerning vertex contributions, are even responsible for the non-unitarity of the theory and for UV/IR mixing. In other words UV divergences are not cured but rather are accompanied by surprisingly emergent IR ones.

The key point of the origin of these malicious features, is basically related to perturbative expansion in $\theta$ of the above action, which is the unique viable procedure to extract phenomenologically testable results from the formal field theoretical apparatus. As a result any truncation at a desired order in the noncommutative parameter basically destroys the non-locality encoded in (5) and gives rise to a local field theory, plagued by spurious momentum-dependent interactions. In other words the desired non-locality induced by the noncommutative manifold is connected to the presence of infinite derivative terms in the product of functions. For this reason any sort of expansion in $\theta$ provides a local field theory, which has nothing to do, even remotely, with the original non-local field theory. Only recently, have there been attempts to restore unitarity 126 and to overcome the problem of UV/IR mixing $127[128$, even if the restriction of noncommutative corrections only to interaction terms remains a non intuitive feature. Against this background, the most efficient way out is that of a radical change of perspective about the employment of noncommutative coordinates and the resulting algebra $\mathcal{A}_{\theta}$.

Indeed, to proceed in this direction, we have to invoke the lesson of quantum mechanics: any quantum operator $A$ is subject to fluctuations due to the fuzziness of the quantum phase space, induced by the Heisenberg (non) commutation relations. Therefore, the operator eigenvalues are the unique physically admissible outcomes of a measure of the observable represented by $A$ and the mean value $\langle A\rangle$ of these eigenvalues provides what is more reminiscent of the corresponding classical observable. Indeed mean values, being statistical mixtures of eigenvalues, do take into account the quantum fluctuations of the observable and evolve according to laws which resemble the classical dynamical equations. In this spirit, to extract some physically meaningful content from the noncommutative algebra $\mathcal{A}_{\theta}$, one has to consider the set of continuous real functions, which corresponds the mean values $\langle\hat{f}\rangle$ of each element $\hat{f} \in \mathcal{A}_{\theta}$. This procedure lets us work with conventional multiplication laws because mean values preserve the non-local character encoded in the noncommutative nature of the algebra $\mathcal{A}_{\theta}$. Thus, the problem is to find proper non- 
commutative states to calculate mean values $\langle\hat{f}\rangle$ : starting from a noncommutative relation (5), there are no coordinate eigenstates for the coordinate operators and no coordinate representation can be defined. Pioneering works 129 in quantum optics and referred to phase space, suggest that coherent states, properly defined as eigenstates of ladder operators built from noncommutative coordinate operators only, are the closest to the sharp coordinate states, which we can define for noncommutative coordinates. In other words, coordinate coherent states are the minimal uncertainty states over the noncommutative manifold and let us calculate the aforementioned mean values.

As an example of the action of mean values on the noncommutative algebra $\mathcal{A}_{\theta}$, let us consider the simplest noncommutative manifold, i.e. the noncommutative plane ${ }^{130 \mid 132}$ embedded in a $2+1$ dimensional spacetime, in which only spatial coordinates are noncommutative

$$
\left[\mathbf{x}^{i}, \mathbf{x}^{j}\right]=i \theta \epsilon^{i j}
$$

with $i, j=1,2$, while $\theta^{i j}=\theta \epsilon^{i j}$ can be written in terms of a unique noncommutative parameter $\theta$ and the anti-symmetric tensor $\epsilon^{i j}$. The relation (9) tells us that we cannot build eigenstates $\left|x^{1}, x^{2}\right\rangle$ and therefore one is led to consider the operators $\mathbf{z}$ and $\mathbf{z}^{\dagger}$ in order to define a convenient set of states

$$
\begin{aligned}
\mathbf{z} & \equiv \frac{1}{\sqrt{2}}\left(\mathbf{x}^{1}+i \mathbf{x}^{2}\right) \\
\mathbf{z}^{\dagger} & \equiv \frac{1}{\sqrt{2}}\left(\mathbf{x}^{1}-i \mathbf{x}^{2}\right)
\end{aligned}
$$

satisfying the relation $\left[\mathbf{z}, \mathbf{z}^{\dagger}\right]=\theta$. The advantage of shifting the noncommutative character from $\mathbf{x}^{1}, \mathbf{x}^{2}$ to $\mathbf{z}$ and $\mathbf{z}^{\dagger}$ consists in the possibility of having eigenstates

$$
\begin{gathered}
\mathbf{z}|z\rangle=z|z\rangle \\
\langle z| \mathbf{z}^{\dagger}=\langle z| z^{*}
\end{gathered}
$$

where $|z\rangle \equiv \exp \left(-\frac{z z^{*}}{2 \theta}\right) \exp \left(-\frac{z}{\theta} z^{\dagger}\right)|0\rangle$, with $|0\rangle$ the vacuum state annihilated by $\mathbf{z}$ and $z$ the complex eigenvalues. These coordinate coherent states $|z\rangle$ allow us to associate to any quantum operator $F\left(\mathbf{x}^{1}, \mathbf{x}^{2}\right)$ the corresponding function $F(z) \equiv\left\langle z\left|F\left(\mathbf{x}^{1}, \mathbf{x}^{2}\right)\right| z\right\rangle$ representing its mean value over the fluctuating manifold, in terms of quasi classical coordinates or simply quasi coordinates, namely mean values $x^{1}, x^{2}$ of coordinate operators

$$
\begin{gathered}
\left\langle z\left|\mathbf{x}^{1}\right| z\right\rangle=\sqrt{2} \Re z \equiv x^{1} \\
\left\langle z\left|\mathbf{x}^{2}\right| z\right\rangle=\sqrt{2} \Im z \equiv x^{2} .
\end{gathered}
$$

These definitions are the prelude to the quantum field theory on the noncommutative plane

$$
\phi(t, z)=\sum_{E, p}\left[\mathbf{a}_{p}^{\dagger} e^{-i E t}\left\langle z\left|\exp \left(i p_{j} \mathbf{x}^{j}\right)\right| z\right\rangle+\text { h.c. }\right]
$$


where $\mathbf{a}_{p}, \mathbf{a}_{p}^{\dagger}$ are the conventional lowering/rising operators acting on Fock states with definite energy and momentum. The novelty of the above definition lies in $\left\langle z\left|\exp \left(i p_{j} \mathbf{x}^{j}\right)\right| z\right\rangle$, which introduced a gaussian damping factor $e^{-\theta p^{2} / 4}$. In other words, the noncommutativity of coordinates manifests as a modification of the integration measure in momentum space, once ordinary finite functions like $F(z)$ are represented by their inverse Fourier transform. An illuminating feature of this noncommutative behavior is given by the form of the momentum space free propagator

$$
G\left(E, \vec{p}^{2}\right)=\frac{e^{-\theta p^{2} / 4}}{-E^{2}+\vec{p}^{2}+m^{2}}
$$

which elegantly exhibits the sought ultraviolet behavior. As a consequence the corresponding Green's function equation exhibits a modified source term

$$
\left(\triangle+m^{2}\right) G_{\theta}(\vec{x}-\vec{y})=\rho_{\theta}(\vec{x}-\vec{y})
$$

namely the conventional Dirac delta has been replaced by a Gaussian distribution, the signature of the pointless geometry.

The nice feature of this approach is that the noncommutative fluctuations of the manifold produce an ultraviolet damping factor in the Fourier transform of field functions defined over it, even at the level of a free field, independently of their tensorial nature and preserving the conventional point-wise product among them. Important progress in this area has been made in order to extend the above two dimensional toy model to a full noncommutative field formulation, in which all of the coordinates are noncommutative: as a consequence the resulting field theory is UV finite, free from any IR contribution, while unitarity and Lorentz invariance are recovered if one imposes a single noncommutative parameter $\theta$ in the theory, namely

$$
\left[\mathbf{x}^{\mu}, \mathbf{x}^{\nu}\right]=i \theta \sigma^{\mu \nu}
$$

where $\sigma^{\mu \nu}$ is an antisymmetric dimensionless tensor determined in Ref. 121 .

The effective quantum description of the physics of radiating black holes still needs a lot of work. First of all, one has to extend the flat space noncommutative formulation in order to include the gravitational interaction: indeed the modification of gravity due to the introduction of (3) followed two distinct paths, which are mainly based on the two formalisms which we have just introduced. Therefore, we will present the resulting noncommutative equivalent of a black hole and we will try to understand the role of $\theta$ versus the Plank phase of the evaporation in both the above approaches.

\section{Black Hole Solutions in Noncommutative Gravity}

The formulation of a full consistent noncommutative version of General Relativity is a business of primary interest and currently the subject of a vast literature. Indeed once a model of Noncommutative Geometry is assumed, we would like to know the 
deformation of the gravitational field equations due to the fuzziness of the manifold. At present, in spite of the promising work in this field $133-150$, we are still far from a widely acknowledged theory of Noncommutative Gravity, which, in some sense, is expected to provide the transition from the smooth differential to the stringy (or loopy) picture of spacetime. In this review, we shall not enter into the debate about the accuracy of the proposed models of Noncommutative Gravity in literature, but we will present the formulation which concretely led to new metrics, obtained by solving, exactly or approximately, the noncommutative version of Einstein's equation, in order to study the physically reliable effect in the physics of the resulting black holes. To this purpose, a key point of all noncommutative formulations will be the definition of the equivalent of the line element: indeed, any differential displacement connecting two infinitesimally close spacetime events appears not well defined in a pointless geometry. Furthermore, in presence of the noncommutative fuzziness, we do expect some sort of removal of singularities which occur in the conventional differential spacetime manifold. The latter point can be really considered as the quality certificate which justifies the employment of any Noncommutative Geometry model and discriminate the efficiency of the mathematical machineries.

\subsection{The noncommutative equivalent of the Schwarzschild metric}

We start with the noncommutative corrections to the Schwarzschild metric, proceeding along the line suggested by Ref. 138, namely employing a deformation of Einstein's equation induced by gauging the noncommutative $I S O(3,1)$ groupl. More concretely, one has to consider the gauge field strength of the noncommutative gauge group $I S O(4,1)$ and the contraction to the group $I S O(3,1)$ to determine the deformed vierbein in terms of the undeformed ones. After this stage, one can construct the deformed curvature scalar

$$
\int d^{4} x \sqrt{\hat{e}} \star \hat{e}_{\star a}^{\mu} \star \hat{R}_{\mu \nu}^{a b} \star\left(e_{\star b}^{\nu}\right)^{\dagger} \star \sqrt{\hat{e}}^{\dagger}
$$

where $\hat{e}_{\mu}^{a}$ is the deformed vierbein, while $\hat{e}=\operatorname{det}\left(\hat{e}_{\mu}^{a}\right)$ and the inverse vierbein $\hat{e}_{\star a}^{\mu}$ is given by $\hat{e}_{\star a}^{\mu} \star \hat{e}_{\mu}^{b}=\delta_{a}^{b}$. Here the $\star$-product has been extended in order to be invariant under diffeomorphism transformations. The explicit form of the above quantities can be obtained as an expansion in $\theta$

$$
\begin{aligned}
\hat{e}_{\mu}^{a} & =e_{\mu}^{a}+i \theta^{\nu \rho} e_{\mu \nu \rho}^{a}+\theta^{\nu \rho} \theta^{\kappa \sigma} e_{\mu \nu \rho \kappa \sigma}^{a}+O\left(\theta^{3}\right) \\
\hat{e}_{\star a}^{\mu} & =e_{a}^{\mu}+i \theta^{\nu \rho} e_{a \nu \rho}^{\mu}+\theta^{\nu \rho} \theta^{\kappa \sigma} e_{a \nu \rho \kappa \sigma}^{\mu}+O\left(\theta^{3}\right)
\end{aligned}
$$

where $e_{\mu \nu \rho}^{a}$ and $e_{\mu \nu \rho \kappa \sigma}^{a}$ can be written in terms of $e_{\mu}^{a}, \theta^{\mu \nu}$ and the spin connection $\omega_{\mu}^{a b}$. As a result, in Ref. 151, the corresponding deformed metric has been introduced, assuming

$$
\hat{g}_{\mu \nu}=\frac{1}{2} \eta_{a b}\left(\hat{e}_{\mu}^{a} \star \hat{e}_{\nu}^{b \dagger}+\hat{e}_{\mu}^{b} \star \hat{e}_{\nu}^{a \dagger}\right)
$$

iThe $I S O(3,1)$ group is the full Poincare group, including both the elements of $S O(3,1)$ and spacetime translations. 
where $\hat{e}_{\mu}^{a}$ are written in terms of the conventional Schwarzschild vierbein by means of an expansion in $\theta$. Therefore the resulting deformed Schwarzschild metric $\hat{g}_{\mu \nu}$ has the following nonzero components

$$
\begin{aligned}
& \hat{g}_{00}=g_{00}-\frac{\alpha(8 r-11 \alpha)}{16 r^{4}} \theta^{2}+O\left(\theta^{4}\right) \\
& \hat{g}_{11}=g_{11}-\frac{\alpha(4 r-3 \alpha)}{16 r^{2}(r-\alpha)^{2}} \theta^{2}+O\left(\theta^{4}\right) \\
& \hat{g}_{22}=g_{22}-\frac{2 r^{2}-17 \alpha r+17 \alpha^{2}}{32 r(r-\alpha)} \theta^{2}+O\left(\theta^{4}\right) \\
& \hat{g}_{33}=g_{33}-\frac{\left(r^{2}+\alpha r-\alpha^{2}\right) \cos \psi-\alpha(2 r-\alpha)}{16 r(r-\alpha)} \theta^{2}+O\left(\theta^{4}\right)
\end{aligned}
$$

where $\alpha=2 G M / c^{2}$ and $g_{\mu \nu}$ is the known Schwarzschild metric. As one can see, the singular behavior at the origin of the Schwarzschild manifold is unaffected by the noncommutative corrections, but it is rather accompanied by even worse $1 / r^{4}$ terms.

Another kind of deformation of gravity is based on the requirement that $\theta^{\mu \nu}$ is a covariantly constant tensor in curved space and that the symmetries of spacetime reduce to volume-preserving diffeomorphism which also preserve $\theta^{\mu \nu}$. The consequences of such proposal have been analyzed by means of an extension of both Moyal and Kontsevich product 152 i to curved space and lead to the noncommutative contribution to the metric at the linearized leve 153

$$
\begin{aligned}
& g_{00}=1-\frac{2 G M}{r}\left(1+3 G \vec{\theta}^{2}-G(\vec{n} \cdot \vec{\theta})^{2}\right) \\
& g_{0 i}=0, \\
& g_{i j}=-\delta^{i j}-\frac{2 G M}{r}\left[n^{i} n^{j}+G \delta^{i j}\left(\vec{\theta}^{2}-(\vec{n} \cdot \vec{\theta})^{2}\right)+G\left(\theta^{i} n^{j}+\theta^{j} n^{i}\right) \vec{n} \cdot \vec{\theta}\right]
\end{aligned}
$$

where $n^{i}=x^{i} / r$ is the radial unit vector, while $\theta^{j}$ is defined in such a way that $\theta^{i j}=\varepsilon^{i j k} \theta^{k}$. As a result, the Newtonian potential has a noncommutative contribution proportional to $\vec{\theta}^{2}$, which can be incorporated into $G$ to give rise to an effective Newton constant, even if no regularization mechanism occurs at the origin. Furthermore, the Newtonian potential also acquires an even worse angular dependent term $(\vec{n} \cdot \vec{\theta})^{2}$, which goes like $1 / r^{2}$, a contribution which appears physically inconsistent.

Recently, another approach 154 has been proposed to calculate noncommutative long-distance correction to the classical geometry, on the ground of semiclassical arguments, namely considering only the corrections due to the interaction of linearized graviton field with noncommutative matter energy momentum tensor

$$
\mathcal{L}_{i n t}=\frac{1}{2} \int d^{4} x h_{\mu \nu} T_{N C}^{\mu \nu}
$$

j The employment of the Kontsevich product let us consider the case in which $\theta^{\mu \nu}$ is no longer constant, but satisfies the Jacobi identity. 
where

$$
T_{N C}^{\mu \nu}=\frac{1}{2}\left(\partial^{\mu} \phi \star \partial^{\nu} \phi+\partial^{\nu} \phi \star \partial^{\mu} \phi\right)-\frac{1}{2} \eta^{\mu \nu}\left(\partial_{\alpha} \phi \star \partial^{\alpha} \phi-m^{2} \phi \star \phi\right) .
$$

The graviton field, for a nearly static source, can be written as the Fourier integral

$$
h_{\mu \nu}(x)=-16 \pi G \int \frac{d^{3} q}{(2 \pi)^{3}} e^{i \vec{q} r} \frac{1}{\vec{q}^{2}}\left(T_{\mu \nu}^{N C}(q)-\frac{1}{2} \eta_{\mu \nu} T_{N C}(q)\right)
$$

where $T_{\mu \nu}^{N C}(q)=\left\langle p_{2}\left|: T_{\mu \nu}^{N C}(x):\right| p_{1}\right\rangle$ and $q_{\mu}=\left(p_{2}-p_{1}\right)_{\mu}$. As usual, the actual calculation is performed truncating the representation of the $\star$-product at a given order, this time to retain terms with four derivative at the most

$$
T_{N C}^{\mu \nu} \approx T_{0}^{\mu \nu}+\eta^{\mu \nu} \frac{m^{2}}{16} \theta^{\alpha \beta} \theta^{\rho \sigma} \partial_{\alpha} \partial_{\beta} \phi \partial_{\rho} \partial_{\sigma} \phi
$$

where $T_{0}^{\mu \nu}$ is the conventional commutative free scalar field energy momentum tensor. The resulting metric reads

$$
\begin{aligned}
& g_{00}=\left[1-2 \frac{G m}{r}+2 \frac{G^{2} m^{2}}{r^{2}}-2 \frac{G^{3} m^{3}}{r^{3}}+\ldots\right]+\delta h_{00}^{N C} \\
& g_{i j}=\left[-\delta_{i j}\left(1+2 \frac{G m}{r}\right)-\frac{G^{2} m^{2}}{r^{2}}\left(\delta_{i j}+\frac{r_{i} r_{j}}{r^{2}}\right)+2 \frac{G^{3} m^{3}}{r^{3}} \frac{r_{i} r_{j}}{r^{2}}+\ldots\right]+\delta h_{i j}^{N C}(36) \\
& g_{0 i}=0
\end{aligned}
$$

where the noncommutative corrections coming from the four derivatives term in the energy momentum tensor are

$$
\begin{aligned}
& \delta h_{00}^{N C}=\frac{G m^{3} \theta^{0 i} \theta^{0 j}}{4 \pi}\left[-\frac{\delta_{i j}}{r^{3}}+\frac{3 r_{i} r_{j}}{r^{5}}\right] \\
& \delta h_{k m}^{N C}=-\delta_{k m} \delta h_{00}^{N C} \\
& \delta h_{0 k}^{N C}=0 .
\end{aligned}
$$

As we can see, a post-post-Newtonian term appears as a result of the noncommutativity, which, at least for the Solar system, would lead to $\sqrt{\theta}>10^{-8} \mathrm{~cm}$, an unlikely lower bound to have significant correction dominating the post-post Newtonian one of the classical theory. A second term in (38) reveals a violation of the Lorentz invariance, even though there are no signals for this feature throughout the formulation and the calculations. Finally the above results are not capable of probing the geometry at the origin and smear out the singularity. This problem seems to persist also in the effective metric emerging in the matrix model framework for noncommutative $U(n)$ gauge theory: even if any conclusion would be premature, preliminary results fatally provide a badly behaving geometry at the origin 155 . As we shall see, the absence of any regularization mechanism is due to the employment of an actually local field theory, rather than to the asymptotic nature of the corrections as it might seem to be at first sight. 
Employing a mere "substitution" of the radial coordinate with $\hat{r} \equiv\left(\sum_{i} \hat{x}_{i}^{2}\right)^{1 / 2}$, where $\hat{x}_{i}$ 's satisfy the noncommutative relation (3), another line element has been recently proposed as possible equivalent of the Schwarzschild one ${ }^{156 \mid 157}$

$$
d s^{2}=\left(1-\frac{2 M}{\hat{r}}\right) d t^{2}-\frac{d \hat{r} d \hat{r}}{\left(1-\frac{2 M}{\hat{r}}\right)}-\hat{r} \hat{r}\left(d \psi^{2}+\sin ^{2} \psi d \phi^{2}\right) .
$$

Writing this line element in terms of the commutative coordinates, $x_{i}=\hat{x}_{i}+\frac{1}{2} \theta_{i j} \hat{p}_{j}$, with $\hat{p}_{i}=p_{i}$, one can determine the corrections due to the above substitution, by a perturbative expansion only

$$
g_{00} \simeq\left(1-\frac{2 M}{r}\right)-\frac{G M}{2 r^{3}}\left[\vec{L} \vec{\theta}-\frac{1}{8}\left(p^{2} \theta^{2}-(\vec{p} \cdot \vec{\theta})\right)\right]+\mathcal{O}\left(\theta^{3}\right)
$$

where $L_{k}=\varepsilon_{i j k} x_{i} p_{j}$, with $p_{i}$ given by $\left[x_{i}, p_{j}\right]=i \delta_{i j}$ and $\vec{\theta}$ by $\theta_{i j}=\frac{1}{2} \epsilon_{i j k} \theta_{k}$. This result is subject to some concern: the noncommutative behavior of spacetime is an exclusive feature of the radial coordinate, through the "substitution" $r \rightarrow \hat{r}$, a fact in contradiction with the covariant nature of the commutative coordinates; there are some ambiguities in the notation since the differential displacement $d s^{2}$ is written in terms of hermitian operators $x^{i}$ and $p^{i}$ rather than functions and nowhere is it defined, $d \hat{r}$ a symbol which appears somehow inconsistent; the line element is no longer a solution of any gravitational field equation, which could support this procedure; the linear and angular momentum terms appear inconsistent, leading $1 / r^{3}$ corrections, which make worse the behavior of the geometry at the origin.

\subsubsection{Towards the noncommutative inspired Schwarzschild black hole}

The analysis which we have made up to now put a severe limitation about the possibility of employing the above metrics for all practical purposes in the field of particle phenomenology, where the Planck phase of the resulting black hole is expected to happen. As we have already highlighted, any perturbative expansion in $\theta$ cannot any longer govern the nonlocal character of the noncommutative manifold and cannot cure the singular behavior of conventional black hole solutions, leading to weakly reliable physical conclusions about the final stage of the evaporation. Against this background, a new perspective has been proposed in 2005, in order to take seriously into account the role of the noncommutative quasi coordinates, obtained by averaging position operators. Indeed in Ref. 158, the modification of the Newtonian potential coming from the $h_{00}$ component of the graviton field

$$
h_{00}(x)=-8 \pi G \int d^{4} y G(x, y) T_{00}(y),
$$

has been for the first time addressed, considering gaussian smearing of both the energy momentum tensor and the propagator in momentum space. Contrary to what we have already discussed about Ref. 154, the calculation has been performed exactly, namely without any sort of expansion in the noncommutative parameter $\theta$. 
The final result leads to the following form of the potential $\phi(r)$

$$
\phi(r)=-G \frac{M}{r} \operatorname{erf}\left(\frac{r}{2 \sqrt{\theta}}\right)
$$

where the error function is defined as $\operatorname{erf}(x) \equiv \frac{2}{\sqrt{\pi}} \int_{0}^{x} d t e^{-t^{2}}$. The asymptotic behaviors of $\phi(r)$ reveal how noncommutativity works: indeed at infinity, namely for $r \gg \sqrt{\theta}$, the new solution matches the conventional $1 / r$ form, while for small distances, namely for $r \ll \sqrt{\theta}$, the appearance of the noncommutative parameter $\theta$ regularizes the divergence in such a way that $\phi(0)=-G \frac{M}{\sqrt{\pi \theta}}$. For the first time $\theta$ has here played the role of minimum length, the sought short distance cut-off descending from the intrinsic non-locality of a field propagating on a noncommutative manifold.

This result opened the road to further investigations on the nature of the gravitational interaction, when a noncommutative manifold is considered. Indeed the form (44) has been exploited to propose the $2 D$ noncommutative equivalent of the Schwarzschild black hole 159 and to determine and solve the linearized noncommutative version of the Einstein equation 160|161. Contrary to what was found in Refs. 153, 154, 155, the weak field regime provides important conclusions about the role of noncommutativity in General Relativity and black hole evaporation. Indeed, noncommutativity, being an intrinsic property of the manifold, does not depend on the curvature; therefore if any effect is produced by noncommutativity it must appear also in the weak field regime. Second, in the considered case of static, spherically symmetric geometry, the expression for the temperature does not depend on the weak field expansion and the new thermodynamic behavior of the black hole can be studied, even before the complete Einstein field equation has been analyzed. The adoption of quasi coordinates in the spirit of Ref. 121 leads to a modified form of the linearized Einstein equation

$$
\begin{aligned}
\vec{\nabla}^{2} g_{00} & =8 \pi G \rho_{\theta}(\vec{x}), \\
\rho_{\theta}(\vec{x}) & =\frac{M}{(2 \pi \theta)^{3 / 2}} \exp \left(-\vec{x}^{2} / 4 \theta\right)
\end{aligned}
$$

providing an effective smearing of the conventional Dirac $\delta$ source term, in agreement with the presence of a minimum length $\sqrt{\theta}$ throughout the manifold. On the other hand, the geometrical part, coming from the Einstein tensor is formally left unchanged, even if one has to keep in mind that this tensor is now written in terms of the aforementioned quasi coordinates.

Therefore one can obtain, without any perturbative expansion in $\theta$, the noncommutative equivalent of the linearized Schwarzschild line element

$$
d s^{2}=\left(1-\frac{2 M G}{\sqrt{\pi} r} \gamma\left(1 / 2, r^{2} / 4 \theta\right)\right) d t^{2}-d l^{2}
$$

where $\gamma$ is the lower incomplete Gamma function, with the definition

$$
\gamma\left(1 / 2, r^{2} / 4 \theta\right) \equiv \int_{0}^{r^{2} / 4 \theta} d t t^{-1 / 2} e^{-t}
$$




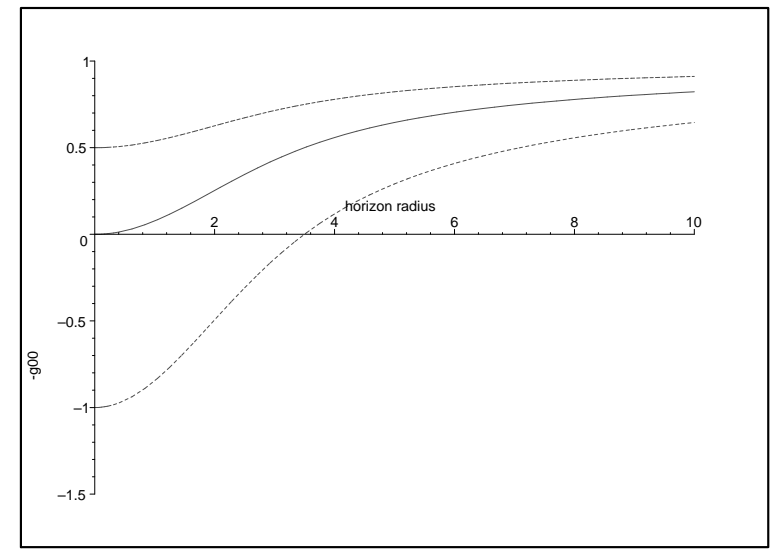

Fig. 1. The linearized solution. The function $g_{00}$ vs the radial distance $r / \sqrt{\theta}$ for some value of the mass $M / \sqrt{\theta} M_{P}^{2}$. The dashed line, corresponds to a mass $M=0.5 M_{0}$ for which there is no event horizon. The dotted line corresponds to a mass $M=2 M_{0}$, which describes a black hole, which is regular at its center $r=0$. The solid curve is the borderline case, namely the case of $M=M_{0}$ in which the horizon radius $r_{H}$ is shrunk to the origin.

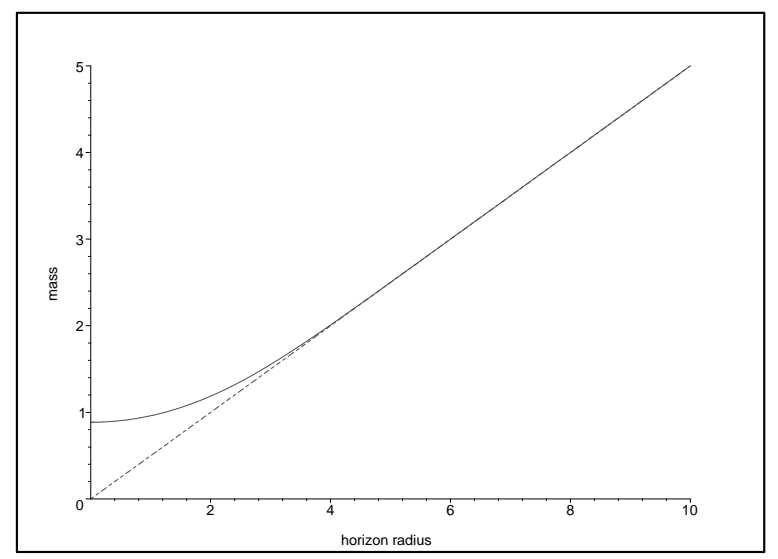

Fig. 2. The linearized solution. The mass $M / \sqrt{\theta} M_{P}^{2}$ vs horizon $r_{H} / \sqrt{\theta}$ relation. In the commutative case, dashed line, the mass is the linear function $M=r_{H} / 2 \ell_{P}^{2}$ vanishing at the origin, while in the noncommutative case, solid line, $M\left(r_{H} \rightarrow 0\right) \rightarrow M_{0}=0.5 \sqrt{\pi \theta} M_{P}^{2}$, i.e. for $M<M_{0}$ there is no event horizon.

Under the condition $r>\sqrt{\theta} \sim \ell_{P}$, the above metric, for its intrinsic nonlocal character, anticipates all the desired features of the noncommutativity, which emerge in the solution of the full Einstein equation: on the geometrical side, the quantum fluctuations of the noncommutative spacetime manifold cure the curvature singularity at the origin; on the thermodynamical side, the resulting black hole undergoes, after a temperature peak, a slowdown of the Hawking emission and ex- 


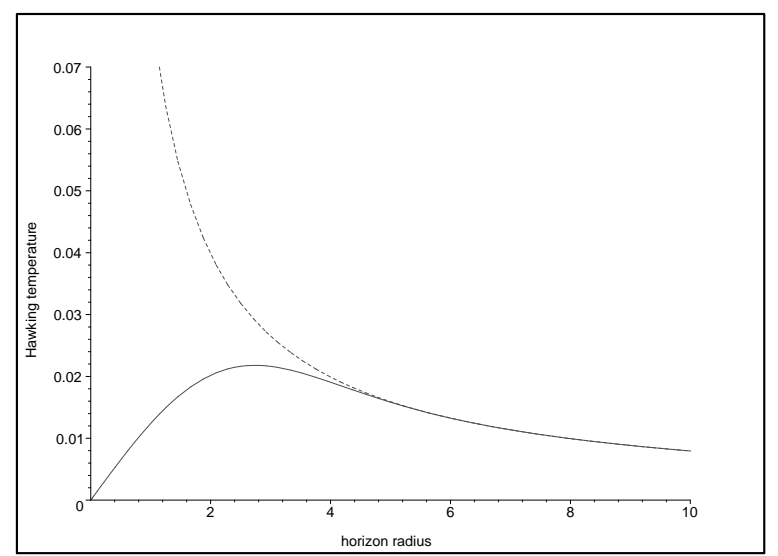

Fig. 3. The linearized solution. The Hawking temperature $T_{H} \sqrt{\theta}$ as a function of the horizon radius $r_{H} / \sqrt{\theta}$. In the noncommutative case, solid curve, one can see that the temperature reaches a maximum value $T_{H}^{M a x} .=2.18 \times 10^{-2} / \sqrt{\theta}$ for $r_{H}=2.74 \sqrt{\theta}$, and then decreases to zero as $r_{H} \rightarrow 0$ (SCRAM phase). The commutative, divergent behavior (Planck phase), dashed curve, is cured.

periences a SCRAM phase $\mathrm{k}$ in place of the Planck phase, shrinking to an absolute zero stable relic. Indeed from the horizon equation (see Fig. 1), one learns that noncommutativity implies a minimum non-zero mass $M_{0}$ in order to have an event horizon (see Fig. 22). Another important aspect is that the above solution is stable, unaffected by any nonlinear perturbation due to back reaction effects. Indeed, preventing a proper Planck phase, noncommutativity keeps the black hole at a state too cool to have a thermal energy comparable with the black hole mass, namely $T / M<2.5 \times 10^{-2} \ell_{P}^{2} / \theta$.

\subsection{The full noncommutative inspired Einstein's equation}

Even if in a simplified form, the above linearized model showed, for the first time, the noncommutativity nicely at work in its regularization business. The need to have more reliable results about the fate of a radiating black hole, namely for $r \sim \sqrt{\theta}$, struck at the time when the spherically symmetric, neutral solution of the full noncommutative inspired Einstein equation was first found, a solution which has been the subject of the seminal paper $\frac{162}{1 n}$ this research area and successfully improved the conventional Schwarzschild geometry. As we shall see, the solution, intentionally called "noncommutative inspired", is subject to a tricky, dual interpretation, often in balance between the classical and the quantum formalism. Indeed the cal-

\footnotetext{
kThe term SCRAM, probably the backronym for "Safety Control Rod Axe Man" or "Super Critical Reactor Axe Man", refers to an emergency shutdown of a thermonuclear reactor. The term has been extended to cover shutdowns of other complex operations or systems in an unstable state, but is also has a "standard" meaning "go away quickly", in particular when we address children or animals.
} 
culations, which we are going to perform, have the appearance of those of classical General Relativity, even if their physical content is purely quantum gravitational. The reason of this can be found in the employment of the noncommutative quasi coordinates, that give rise to a quantum formalism in terms of noncommutative deformed classical functions. Therefore, it will be natural that some features of the solution have their explanation only in terms of Quantum Gravity.

We shall start from some basic considerations about the known Schwarzschild solution, which is often considered a "vacuum solution", since it solves the homogeneous equation $R_{\mu \nu}=0$. Even if, for computational purposes the latter homogeneous equation provides the solution sought, strictly speaking any "vacuum solution" has to be found only in the absence of matter. Indeed, as elegantly shown in Ref.163, the above homogeneous equation $R_{\mu \nu}=0$ works computationally because one implicitly assumes a source term, the energy momentum tensor, concentrated on a region, the origin, excluded from the domain $\mathbb{R} \times\left(\mathbb{R}^{3} \backslash\{0\}\right)$ of the solving line element. As a result, one is led to the physically inconsistent situation in which curvature is generated by a zero energy momentum tensor, while, properly speaking, only the Minkowski spacetime can be considered a "vacuum solution" of Einstein's equation. These considerations are necessary in view of the noncommutative equivalent of the Schwarzschild solution, because in any pointless geometry one cannot employ the shortcut of the homogeneous equation, which is based on the assumption that matter is concentrated in a single point. Indeed we do expect that the energy momentum tensor is diffused throughout a region of linear size governed by the noncommutative parameter $\theta$.

\subsubsection{The noncommutative inspired Schwarzschild solution}

We shall start from the noncommutative version of the Einstein equation in terms of quasi classical coordinates

$$
G_{\theta}^{\mu \nu}=\frac{8 \pi G}{c^{2}} T_{\theta}^{\mu \nu}
$$

in which the only relevant modifications occur in the source term, while $G_{\theta}^{\mu \nu}$ is formally left unchanged. Indeed, considering the energy density distribution of a static, spherically symmetric, noncommutative diffused, particle-like gravitational source, one gets

$$
\rho_{\theta}(r)=\frac{M}{(4 \pi \theta)^{3 / 2}} \exp \left(-r^{2} / 4 \theta\right)
$$

which replaces the conventional Dirac $\delta$ distribution 164 and enters the temporal component of the energy momentum tensor

$$
T_{\theta 0}^{0}=-\rho_{\theta}(r) .
$$


The covariant conservation $T_{\theta}^{\mu \nu} ; \nu=0$ and the additional "Schwarzschild like" condition $g_{00}=-g_{r r}^{-1}$, completely specify the form of the energy momentum tensor

$$
T_{\theta \mu}^{\nu}=\left(\begin{array}{cccc}
-\rho_{\theta} & & & \\
& p_{r} & & \\
& & p_{\perp} & \\
& & & p_{\perp}
\end{array}\right)
$$

with $p_{r}=-\rho_{\theta}$ and $p_{\perp}=-\rho_{\theta}-\frac{r}{2} \partial_{r} \rho_{\theta}(r)$. The above energy momentum tensor is rather unusual because differs from the conventional perfect fluid tensor, which exhibits isotropic pressure terms: on the other hand it is easy to show that $p_{r}$ and $p_{\perp}$ are different only within few $\sqrt{\theta}$ from the origin and the perfect fluid condition is reestablished for larger distances. Plugging the above $T_{\theta}^{\mu \nu}$ into the resulting Einstein equation, we find the line elementl:

$d s^{2}=-\left(1-\frac{4 M}{r \sqrt{\pi}} \gamma\left(3 / 2, r^{2} / 4 \theta\right)\right) d t^{2}+\left(1-\frac{4 M}{r \sqrt{\pi}} \gamma\left(3 / 2, r^{2} / 4 \theta\right)\right)^{-1} d r^{2}+r^{2} d \Omega^{2}$

where $d \Omega^{2}=d \vartheta^{2}+\sin ^{2} \vartheta d \phi^{2}$ and $\gamma\left(3 / 2, r^{2} / 4 \theta\right)$ is the lower incomplete Gamma function

$$
\gamma\left(3 / 2, r^{2} / 4 \theta\right) \equiv \int_{0}^{r^{2} / 4 \theta} d t t^{1 / 2} e^{-t}
$$

The above metric describes a self-gravitating, anisotropic fluid-type matter, whose non vanishing radial pressure is a consequence of the quantum fluctuation of the spacetime manifold and balances the inward gravitational pull, preventing the matter collapsing into a point. This is one of the first physically relevant effects due to noncommutativity: indeed on purely classical grounds, one would be led to believe in a matter collapse, the contrary of what happens when the intrinsic quantum gravitational nature of the solution is taken into account. As expected, the relevance of the noncommutative correction occurs in a neighborhood of the origin i.e. $r \lesssim \theta$, where both the radial and the tangential pressures together with the matter density are regularized by $\theta$ and share a finite value, $M /(4 \pi \theta)^{3 / 2}$. Asymptotically far away from the origin, the density and the pressures virtually vanish, reproducing the regime which supported the name of "vacuum solution". To have a complete understanding of the further surprising features, it is worthwhile to analyze both the geometrical and the thermodynamical behavior of the solution.

\subsubsection{Horizons, curvature and energy conditions}

Let us start from the study of the horizon equation $-g_{00}\left(r_{H}\right)=g^{r r}\left(r_{H}\right)=0$.

Fig. 4illustrates the role of noncommutativity and the new behavior with respect to the conventional Schwarzschild solution: instead of a single event horizon at

${ }^{1}$ We are using the convenient units $G=c=1$. 


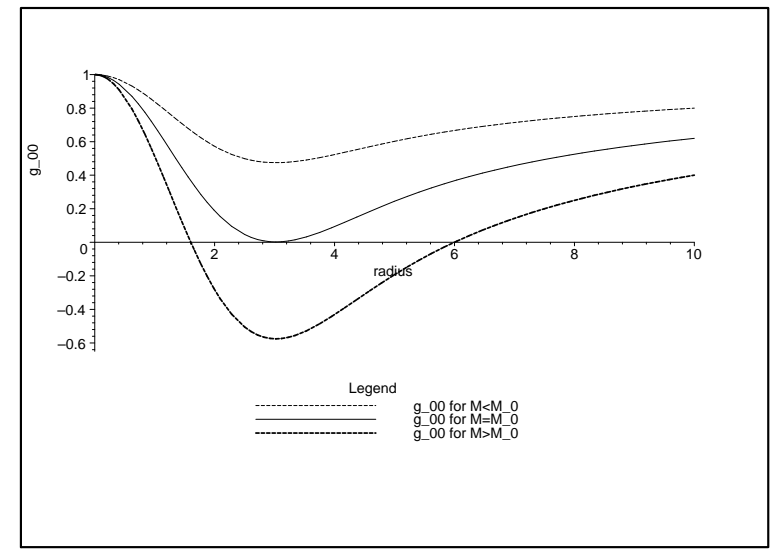

Fig. 4. The noncommutative Schwarzschild solution. The function $-g_{00}=g_{r r}^{-1}$ vs $r / \sqrt{\theta}$, for various values of $M / \sqrt{\theta}$. Intercepts on the horizontal axis give radii of the horizons. The lighter curve corresponds to $M=1.00 \sqrt{\theta}$ (no horizon), the darker curve below corresponds to $M=M_{0} \approx$ $1.90 \sqrt{\theta}$ (one degenerate horizon at $r_{H}=r_{0} \approx 3.0 \sqrt{\theta}$ ) and finally the lowest curve corresponds to $M=3.02 \sqrt{\theta}$ (two horizons at $r_{H}=r_{-} \approx 1.60 \sqrt{\theta}$ and $r_{H}=r_{+} \approx 6.00 \sqrt{\theta}$ ).

$r_{H}=2 M$, the intersection of the function $g_{00}$ with the $r$ axis shows that there is a minimum mass $M_{0} \approx 1.90 \sqrt{\theta}$ below which no event horizon occurs. For $M>M_{0}$ the line element exhibits two distinct horizons, while for the borderline case, $M=M_{0}$, we find a unique degenerate horizon at $r_{H}=r_{0} \approx 3.0 \sqrt{\theta}$, corresponding to the extremal black hole.

As signature of consistency of this noncommutative solution, we find that the curvature singularity at the origin is cured. Indeed the short distance behavior of the Ricci scalar is given by

$$
R(0)=\frac{4 M}{\sqrt{\pi} \theta^{3 / 2}}
$$

Therefore for $r \ll \sqrt{\theta}$, the curvature is constant and positive. Instead of the curvature singularity one finds a deSitter core, whose cosmological constant is governed by noncommutativity, namely $\Lambda=M / 3 \sqrt{\pi} \theta^{3 / 2}$. The presence of such a deSitter core contributes to shedding light on the origin of the finite pressure terms, which we discussed above. In analogy to the appearance of cosmological constant terms induced by quantum vacuum fluctuations, when described in terms of a fluid type energy momentum tensor 165 , the previous pressure terms are the fluid type picture of the quantum noncommutative fluctuations, which determine a regular deSitter core at the black hole center. The above result is in full agreement with previous attempts to match an outer Schwarzschild geometry with an inner deSitter core either through time-like 29|30|166 and space-like shells167|168, attempts which gave rise to the vast literature about regular black holes (for a recent review see Ref. 169 and the references therein). The novelty and the virtue of the above solution lies in the fact that the singularity in not cured placing by hand a regular core at the 
origin, but it is the noncommutative parameter $\theta$ that provides a smooth transition between the inner regular deSitter geometry and the outer Schwarzschild one, without invoking any matching condition. In other words, contrary to other approaches, the regularity of the spacetime is no longer an artifact, but it directly descends from the noncommutative defining relation (5).

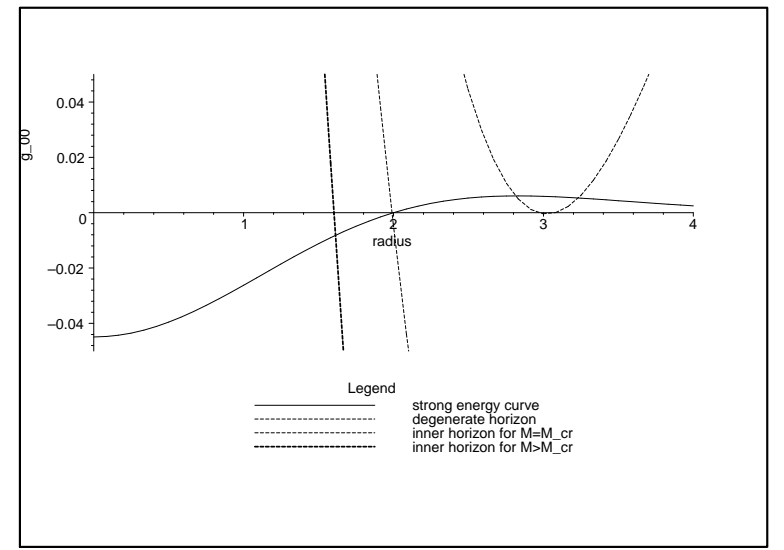

Fig. 5. The noncommutative Schwarzschild solution. The solid curve is the function $\left(\rho_{\theta}+p_{r}+\right.$ $\left.2 p_{\perp}\right) \times \theta$ vs $r / \sqrt{\theta}$. Dashed lines corresponds to $-g_{00}$ for various value of the mass. The intercepts of $\left(\rho_{\theta}+p_{r}+2 p_{\perp}\right)$ with the $r$-axis mark the border, at $r=2 \sqrt{\theta}$, between the classical and quantum description of the spacetime manifold. We observe that for the extremal case, $M=M_{0}$, the degenerate horizon $r_{0}>2 \sqrt{\theta}$ is in the region where the strong energy condition is satisfied. Finally there is the curve of the critical case, namely $-g_{00}$ for a value of the mass $M=M_{c r} \approx 2.35 \sqrt{\theta}$, such that the inner horizon coincides with $2 \sqrt{\theta}$. This means that for $M<M_{c r}$ the classical picture can still be employed to describe the black hole, except in a limited region in the vicinity of the origin, where the classical singular behavior non longer exists.

A very tricky matter regards the energy conditions of the solution. Again, for a correct interpretation of the results, one has to keep in mind the noncommutative origin and the quasi classical procedure, which we have employed up to now. Indeed if we interpret the metric in Eq. (52) merely as a solution of classical Einstein's equation, one would have some concern for the violation of the strong energy condition

$$
\rho_{\theta}+p_{r}+2 p_{\perp} \geq 0
$$

even if the weak energy conditions, $\rho_{\theta}+p_{r} \geq 0$ and $\rho_{\theta}+p_{\perp} \geq 0$ are always satisfied. Indeed spherically symmetric, asymptotically Schwarzschild, regular solutions of this kind have known global structure and are characterized in a precise way: in all generality if they have an (outer) event horizon, they must have an (inner) Cauchy horizon and they fail to be globally hyperbolic. To this purpose, also a nonvanishing matter density distribution beyond the outer horizon is not a novelty and can be considered as a matter of secondary concern. Indeed black holes may be dirty, 
namely they may be surrounded by some kind of matter, admitting the equilibrium as possible configuration $170-177$. On the other hand, as recently underlined 169 , an interesting feature of the noncommutative inspired solution is that the violation of the strong energy condition could take place outside the inner Cauchy horizon, implying a runaway extension of the region where gravity switches to a repulsive quantum interaction. To shed light on this matter it is convenient to note that

$$
\rho_{\theta}+p_{r}+2 p_{\perp} \sim e^{r^{2} / 4 \theta}\left(\frac{r^{2}}{2 \theta}-2\right)
$$

which implies a strong energy condition violation for $r<2 \sqrt{\theta}$. This means that, in spite of the nonlinear nature of gravity, the classical description of matter and energy breaks down only in a region where we supposed, since the beginning, that quantum effects would have been dominant. In other words in the core around the origin, namely within $2 \sqrt{\theta}$ from the black hole center, gravity is actually described by Noncommutative Geometry rather than by General Relativity. For this reason, all the classical arguments cease to be valid at small scales. Anyway, it is interesting to study the extension of the above core around the origin with respect to the position of horizons: to this purpose it is convenient to define $M_{c r}$, the value of the mass parameter for which the region of strong energy violation is confined behind the inner Cauchy horizon. In other words, we have that black holes total with mass $M_{0}<M<M_{c r} \approx 2.35 \sqrt{\theta}$ the breakdown of the classical picture of the solution occurs only in a limited region around the origin, just to cure the singularity thanks to the quantum fluctuations. (see Fig. 5). A related source of concern would be the potential blue shift instability at the inner horizon. Indeed an observer crossing the inner horizon would experience an arbitrarily large blue shift of any incoming radiation and see the entire history of the exterior region in a finite lapse of his own proper time, as he approaches the horizon. This suggested that any small perturbation would disrupt the horizon and develop a curvature singularity. Previous analyses have shown that the inner horizon of the Reissner-Nordström geometry $178-186$, the past horizon of the Schwarzschild white hole $187-189$ and those of the Schwarzschild wormhole 190 are unstable. Even if we do not have yet a definitive answer about this matter, the noncommutative inspired black hole appears to be quite different from the above cases: again the blue shift instability has to be addressed taking into account, that the onset of a curvature singularity is meaningful only for a classical differential manifold. In a noncommutative background, the propagation of any field, representing the perturbation, is subject to the presence of a natural ultraviolet cut-off: therefore no observer could experience an infinite amount of energy, approaching the Cauchy horizon. As a consequence of this, we can conclude that the conventional arguments about the instability of Cauchy horizons should be reviewed, when noncommutative effects are included.

For the special case of absence of event horizons, i.e. $M<M_{0}$, the strong energy condition violation again occurs only in a neighborhood of the origin. In particular, we have an everywhere regular spacetime and no naked singularity appears. The 
resulting regular gravitational system bears a strong resemblance to the gravastar, the hypothetical gravitational vacuum star, whose model is currently at the center of a hot scientific debate 191 . The new feature, introduced by noncommutativity, is that the occurrence of a gravastar is subject to the upper bound on its mass $M<M_{0}$. For this reason we would suggest to call it "mini-gravastar", to distinguish it from its more conventional forerunner. Finally, at the opposite side, i.e. the large mass regime $M \gg M_{0}$, the solution reproduces the standard Schwarzschild geometry, because the inner horizon shrinks to the origin, while the outer horizon coincides with $2 M$, up to negligible exponentially suppressed corrections. The last analysis can be equivalently made also by studying the line element (52) at large distances, i.e. $r \gg \sqrt{\theta}$, a scale at which Noncommutative Geometry turns into General Relativity and effectively describes the gravitational field in terms of a smooth classical manifold.

\subsubsection{The black hole thermodynamics}

The thermodynamics of the noncommutative inspired black hole is even more interesting. In agreement to what has already been seen in the linearized case, the behavior of the temperature reflects the regularity of the manifold. Indeed, also looking at the temperature profile, we can conclude that the black hole is essentially the Schwarzschild one for large distances, where the conventional result $\sim 1 / M$ still holds. The exciting novelty appears, when, during the evaporation, the outer horizon $r_{+}$shrinks to $\sim 6 \sqrt{\theta}$ : the Hawking temperature deviates from the conventional behavior and reaches a maximum at $r_{+}=r_{\text {max }} \approx 4.76 \sqrt{\theta}$, after which the black hole cools down, with a relevant slow down of thermal emission. Thus, the SCRAM is over at $r_{+}=r_{0} \approx 3.02 \sqrt{\theta}$, when the black hole reaches the extremal configuration with mass $M_{0} \approx 1.90 \sqrt{\theta}$, the temperature is zero and the Hawking emission breaks off. Analytically one can determine

$$
T_{H}=\frac{1}{4 \pi r_{+}}\left[1-\frac{r_{+}^{3}}{4 \theta^{3 / 2}} \frac{e^{-r_{+}^{2} / 4 \theta}}{\gamma\left(3 / 2 ; r_{+}^{2} / 4 \theta\right)}\right]
$$

where the mass $M$ is written in terms of $r_{+}$from the horizon equation. Again for $r_{+}<r_{0}$ we cannot speak of an event horizon and no temperature can be defined. To this purpose, the final zero temperature configuration can be considered a black hole relic, namely the remnant of the black hole evaporation via Hawking emission. Such a relic could provide a sort of solution to the long standing problem of the information paradox 192: indeed, due to occurrence of the cool SCRAM phase in place of the hot Planck phase, the initial information is confined and preserved into the black hole relic, even after the end of the evaporation. A detailed analysis of spin-2 quantum amplitudes led to the conclusion that unitarity is preserved during the life of the black hole 193 . From Fig. [6 it follows that the black hole is "colder" with respect to what one would imagine: indeed, even if the maximal temperature could reach the astonishing value $\sim 2.1 \times 10^{30} \mathrm{~K}$, it is not able to prime any back reaction effect. This is one of the key points of the SCRAM phase, which lets us 
employ the solution obtained during all of the black hole's life: in fact to have significant back reaction, one should have $E_{\max } \sim M$, where $E_{\max }$ is the peak of the thermal energy, a condition which leads to $\sqrt{\theta} \lesssim 10^{-34} \mathrm{~cm}$, an unphysical constraint that can never be met.

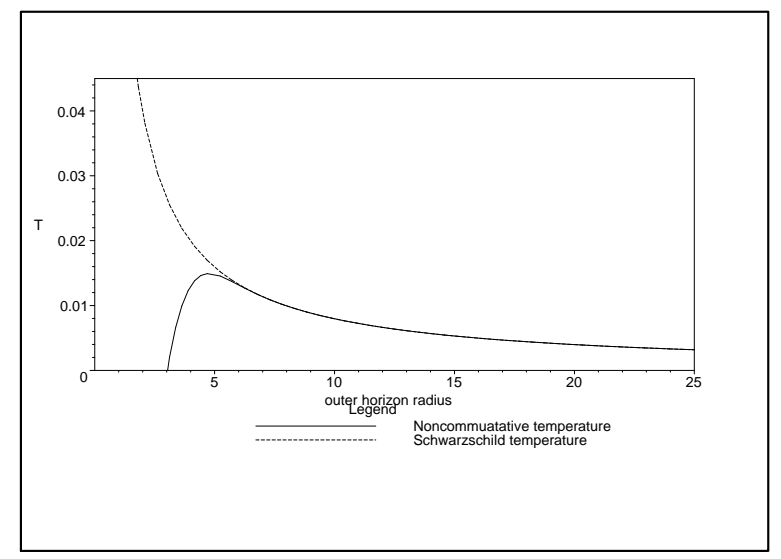

Fig. 6. The noncommutative Schwarzschild solution. The Hawking temperature $T \times \sqrt{\theta}$ vs the outer horizon radius $r_{+} / \sqrt{\theta}$. The dashed curve refers to the conventional result for the Schwarzschild black hole, with a divergent Planck phase in the final stage of the evaporation. The solid curve is the new behavior of the temperature due to noncommutative effects: for $r_{+} \gtrsim 6 \sqrt{\theta}$, the temperature follows the conventional behavior, while it reaches a maximum $T_{H} \simeq 0.015 \times 1 / \sqrt{\theta}$, at $r_{+}=r_{\max } \approx 4.76 \sqrt{\theta}$, corresponding to a mass $M=M_{\max } \simeq 2.4 \times \sqrt{\theta}$. Finally there is a SCRAM phase, leading to $T_{H}=0$ for $r_{+}=r_{0}=3.02 \sqrt{\theta}$, corresponding to a black hole remnant, the extremal black hole configuration.

Regarding the remaining thermodynamical quantities, several authors 194202 have studied the effects of noncommutativity on the laws regulating black holes. In particular, much effort has been recently devoted to the entropy area law, employing the second law of thermodynamics

$$
d S_{H}=\frac{1}{T_{H}} \frac{d M\left(r_{+}\right)}{d r_{+}} d r_{+}
$$

where the function $M\left(r_{+}\right)=\Gamma(3 / 2) r_{+} / 2 \gamma\left(3 / 2 ; r_{+}^{2} / 4 \theta\right)$ is given by the horizon equation $-g_{00}\left(r_{+}\right)=g^{r r}\left(r_{+}\right)=0$. Once integrated from the extremal horizon $r_{0}$ to a generic outer horizon $r_{+}$, the above formula provides that, once integrated from the extremal horizon $r_{0}$ to a generic outer horizon $r_{+}$, provides

$$
\Delta S_{H}=\frac{1}{4 G_{\theta}(r)}\left(A_{+}-A_{0}\right)+\delta S_{H}
$$

where $A_{+}=4 \pi r_{+}^{2}, A_{0}=4 \pi r_{0}^{2}$, while the $G_{\theta}(r)$ is an effective Newton constant $m$

${ }^{\mathrm{m}}$ The line element (52) can be written as

$$
d s^{2}=-\left(1-2 M G_{\theta} / r\right) d t^{2}+\left(1-2 M G_{\theta} / r\right)^{-1} d r^{2}+r^{2} d \Omega^{2}
$$


given by

$$
G \longrightarrow G_{\theta}(r) \equiv G \frac{2}{\sqrt{\pi}} \gamma\left(\frac{3}{2}, \frac{r^{2}}{4 \theta}\right)
$$

and $\delta S_{H}$ is a small correcting term. The first term corresponds to the noncommutative version of the famous Bekenstein-Hawking area law, while further noncommutative corrections are always exponentially small. Indeed, since $r_{0}>\sqrt{\theta}$, we can approximate $\gamma\left(3 / 2, r^{2} / 4 \theta\right) \approx \sqrt{\pi} / 2$ and find

$$
\delta S_{H} \approx \frac{1}{2 \sqrt{\theta} G}\left(r_{0}^{3} e^{-r_{0}^{2} / 4 \theta}-r_{+}^{3} e^{-r_{+}^{2} / 4 \theta}\right) .
$$

We conclude that the area law is maintained up to exponentially small corrections. In support of the above conclusion, there is Fig. 7 we can see that the conventional area-entropy relation holds until $r_{+} \sim r_{\max } \approx 4.76 \sqrt{\theta}$, where the maximal temperature takes place and the SCRAM phase starts. Therefore noncommutative corrections are dominant in the region $r_{0} \lesssim r_{+} \lesssim r_{\max }$. The behavior of the entropy remains qualitatively equivalent to that of the Schwarzschild case at least with respect to the Third Law of Thermodynamics: the entropy near to absolute zero is governed only by the temperature and tends to a constant minimum value independently of the other parameters. In particular we have $\Delta S_{H}=0$, for the extremal black hole relic at $r_{+}=r_{0}$ (see Refs. 195, 200, 202).

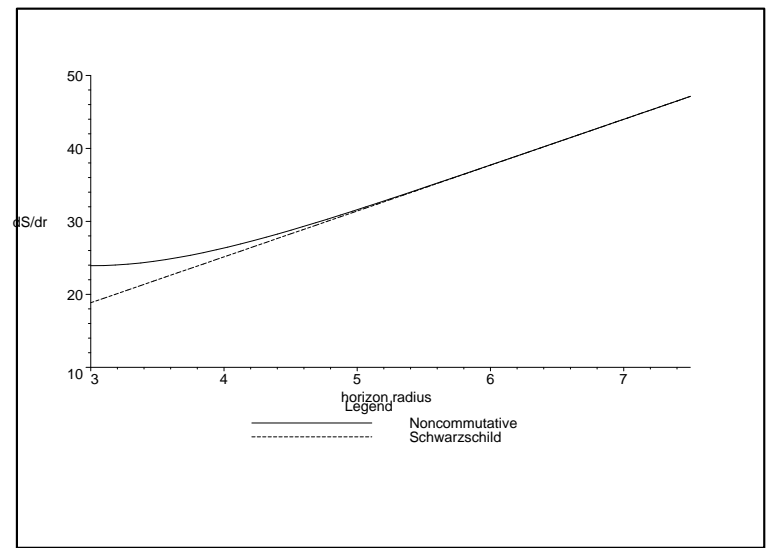

Fig. 7. The noncommutative Schwarzschild solution. The function $d S_{H} / d r_{+} \times \sqrt{\theta}$ vs the horizon radius $r_{+} / \sqrt{\theta}$. Deviations from the conventional linear behavior occur around the $r_{+} \approx 4.76 \sqrt{\theta}$, corresponding to the temperature peak and the beginning of the SCRAM phase.

resolving the long standing problem of an asymptotically safe gravitational coupling 203. Contrary to what was found in Ref. 204 the short distance behavior of the incomplete gamma function implies $G_{\theta} \sim r^{3}$ for small $r$, curing the curvature singularity without any artificial introduction of an ad hoc smoothing function $d(r)$. 


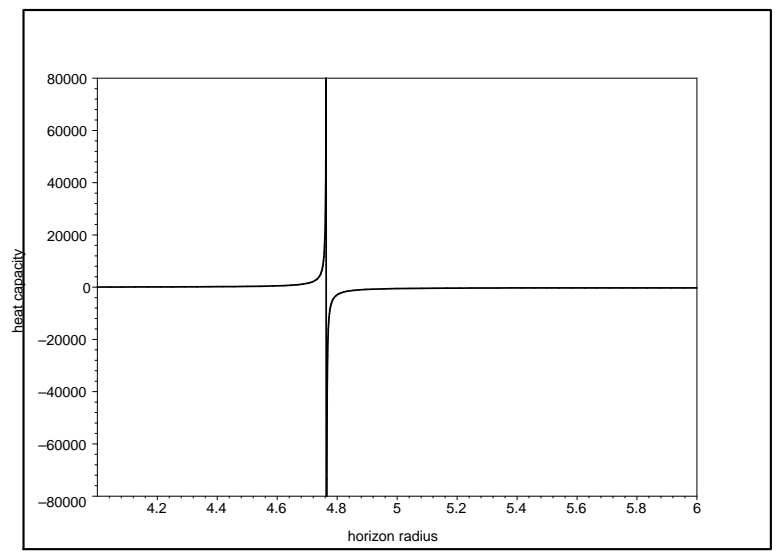

Fig. 8. The noncommutative Schwarzschild solution. The heat capacity $C \times \theta$ vs $r_{+} / \sqrt{\theta}$.

A further inspection into the role of noncommutativity can be made by looking at the black hole heat capacity $C \equiv d M / d T_{H}$, that can be written as

$$
C\left(r_{+}\right)=T_{H}\left(\frac{d S_{H}}{d r_{+}}\right)\left(\frac{d T_{H}}{d r_{+}}\right)^{-1}
$$

This relation highlights the black hole exchanges of energy with the environment. Any zero for the temperature corresponds to $C=0$, leading to the conclusion that the black hole relic is a thermodynamically stable object, that cannot exchange energy and evaporate. On the other hand, any zero in $d T_{H} / d r_{+}$produces a pole and marks a change of sign of the heat capacity, switching the black hole to a $C>0$ stable system, namely the very beginning of the SCRAM phase. The occurrence of a divergent behavior of the heat capacity should not be worrying, since it is only an artifact in the definition of $C$. To this purpose, in support to the consistency of the procedure, it is worthwhile to see that, even at critical points of $C$ and $T_{H}$, the entropy

$$
S_{H}=C\left(T_{H}\right)\left(\frac{d T_{H}}{T_{H}}\right)
$$

is always a positive finite function, giving correct black hole thermodynamics. Therefore the infinite discontinuity of the heat capacity could signal the presence of some sort of phase transition between a Schwarzschild unstable regime and a SCRAM stable regime $205 \mid 206$. Finally we introduce the black hole free energy

$$
F\left(r_{+}\right)=M\left(r_{+}\right)-T_{H}\left(r_{+}\right) S_{H}\left(r_{+}\right)
$$

whose variations reveal, for $\delta F>0$ spontaneous processes or, for $\delta F<0$, disfavored processes or even equilibrium states, for $\delta F=0$. Therefore it is worthwhile to study the behavior of $d F / d r_{+}$, which is zero when either $T_{H}$ or $d T_{H} / d r_{+}$are zero. A deeper inspection can be made looking at second derivatives in the critical 
points: to this purpose, numerical estimates 195 confirm that, as expected, the free energy has a minimum at $r_{+}=r_{\max } \approx 4.76 \sqrt{\theta}$. As a consequence, $d F / d r_{+}$is positive for $r_{0} \leq r_{+} \leq r_{\max }$, because of the positive heat capacity which characterize the SCRAM phase. This means that the evaporation turns into a disadvantageous process, slowing down in the vicinity of the zero temperature configuration.

\subsubsection{Black hole lifetime and detection}

Another important aspect of the black hole thermodynamics is the time scale of the evaporation. On the basis of the above considerations, we can assume that this process takes place essentially in two phases, the Schwarzschild and the SCRAM phase, before and after the temperature peak respectively. The formula governing the black hole evaporation rate is

$$
\frac{d M}{d t}=-A_{+} \Phi
$$

where $\Phi$ is the flux of thermal energy emitted by the evaporating black hole. Even if, in the absence of significant back reaction, the integration of the above equation holds for all of life of the black hole, it requires much attention in particular in the vicinity of the final zero temperature state. Indeed from a thermodynamical point of view, approaching the absolute zero would take an infinite time. On the other hand the temperature breaks off beforehand, namely when the black hole reaches the thermal equilibrium with the environment at $T_{H}=T_{C M B} \approx 2.7 \mathrm{~K}$. For these reasons, we do expect a finite black hole life. It is also interesting to have a preliminary estimate of this life, just to fix the time scale. To this purpose a rough calculation of the time it takes the black hole to shrink from an initial configuration to $r_{+}=r_{\max }$, can be made by means of

$$
\frac{d M}{d t} \sim-4 \pi r_{+}^{2} T_{H}^{4}
$$

Therefore the life of the black hole is almost in agreement with the conventional results, namely

$$
\text { Life } \sim 10^{59}\left(\frac{M_{i n}-M_{\max }}{M_{\odot}}\right)^{3} \text { Gyr }
$$

where $M_{\text {in }}$ is the black hole initial mass, $M_{\max } \approx 2.4 \sqrt{\theta} M_{P} / \ell_{P}$ is the mass at the temperature peak and $M_{\odot}$ is the solar mass. As expected, the evaporation produces a significant lowering of the black hole mass only for very tiny initial masses. For this reason, our results would be in agreement with earlier proposal of the elementary black holes207, namely very small black holes, whose mass would be of the order of the Planck mass, emerging as residue of the evaporation. In our scenario, the stability of such small objects208-210, previously called maximo $\rrbracket$, is regained since

${ }^{\mathrm{n}}$ Other names are Friedmons, Cornucopions, Planckons and Informons, even if there are slight but nontrivial differences among them 211 . 
the evaporation no longer occurs at zero temperature. The nature of maximos is rather striking: they have a very small cross section $\sim 10^{-66} \mathrm{~cm}^{2}$ and a large mass $\sim 10^{28} \mathrm{eV}$ making their direct observation difficult. On the other hand, due to their small interaction with ordinary matter, maximos are thought to act like dark matter and be a considerable part of the matter in the Universe $212 \mid 214$. In our scenario, a possible solution to the problem of their observation would be the detection of their thermal emission before its breaking off at the zero temperature, but again one has to deal with unreachable energy scales $\left(\lesssim 1.5 \times 10^{26} \mathrm{eV}\right)$. Regarding the thermodynamics, the properties of such black hole remnants has been extensively studied 215-223, but on a modern perspective, their role can be revitalized adopting additional space-like dimensions, with consequent lowering of energy scales 224 .

Regarding the applications of the noncommutative inspired solution, we mention the description of the dual effect of noncommutativity in both the matter and geometry sector, adopting the $r-t$ section of metric (52) in the world sheet of the 2D bosonic Polyakov string, governed by a noncommutative anomaly induced effective action. The vacuum expectation value of the energy momentum tensor of the resulting 2D scalar field theory in the Boulware, Hartle-Hawking and Unruh vacua has been explicitly calculated: as expected the standard short distance divergences were regularized by the presence of quantum coordinate fluctuations 225 , improving previously known results226-233.

Finally on the more phenomenological side, we know for certain that noncommutative effects are not visible until the electroweak scale, therefore we can safely assume $\sqrt{\theta}<10^{-16} \mathrm{~cm}$. On the opposite limit, the noncommutative correction to the planets perihelion precession of the solar system has been recently evaluated 234 . This could lead to a stringent lower limit on $\sqrt{\theta}$ on the grounds of astronomical data. Anyway, we can safely consider $\sqrt{\theta} \gtrsim 10^{-33} \mathrm{~cm}$. This striking difference of possible scales, is another feature of the still unsolved hierarchy problem. To this purpose, it is clear that with the neutral four dimensional noncommutative inspired solution, we are only scratching the surface of a gold mine. Far more important consequences of noncommutativity will emerge from the extradimensional solutions, in which we may consider as unique scale $1 / \sqrt{\theta} \sim M_{*} \sim 1 \mathrm{TeV}$. A detailed analysis of the extradimensional solutions will be the subject of one of the next chapters.

\section{Noncommutative Charged Black Holes}

A logic step forward in this review is to consider the natural extension of the above neutral solutions in order giving the resulting black hole "Abelian hair", represented by a long range electric field. Apart from a matter of completeness, this generalization is motivated by the understanding of the role of noncommutativity in those phases preceding the Schwarzschild phase: indeed it is vital to know whether the neutralization of the hole still occurs in short times with respect its total mass loss, leading to a neutral zero temperature remnant configuration. On the other hand this program requires a preliminary inspection of noncommutative electrodynamics 
and the analysis of an emerging new decaying channel, i.e. the Schwinger pair production, that, together with the Hawking emission, contributes to the black hole mass loss. As we shall see, the determination of noncommutative charged solutions is crucial also in view of the possible detection of some evidence for mini black holes as a result of the forthcoming extreme energy experiments at LHC: it is very likely that the initial hadronic charge will be distributed among the fragments after the collision, fragments which could include a mini black hole. Therefore, a charged mini black hole could be produced surrounded by a cloud of pairs, repelling those particles of the its own sign. In other words, the presence of many electrons near one of the hadronic fragments would be a potential signal for the presence of a charged mini black hole.

We start along the lines suggested in Ref. 138 to have the extension of the result obtained in Ref. 151, The procedure presented in Ref. 235, 236 is based on the quest for a solution of the following equation

$$
\tilde{R}_{\mu}^{\nu}-\frac{1}{2} \tilde{R} \delta_{\mu}^{\nu}=-T_{\mu}^{\nu}
$$

Here $T_{\mu}^{\nu}$ is the electromagnetic energy momentum tensor, while the curvature tensors are obtained by contraction of the deSitter gauge group $\mathrm{SO}(4,1)$ into the ISO $(3,1)$ Poincaré group, setting the torsion to zero and employing the SeibergWitten map $\frac{108}{10}$ get the noncommutative character of the theory. As a result the representation of the noncommutative deformed vielbein is obtained by expanding the $\star$-product and it is found to be coincident with that already seen in (22) for the Schwarzschild case. As a result, one can compute the resulting deformed metric by the formula (23) to obtain the following non-vanishing components

$$
\begin{aligned}
\hat{g}_{00}= & g_{00}-\frac{1}{r^{6}}\left[M r^{3}-\frac{11 M^{2}+9 Q^{2}}{4} r^{2}-\frac{17 M Q^{2}}{4} r-\frac{7 Q^{4}}{2}\right] \theta^{2}+O\left(\theta^{4}\right) \\
\hat{g}_{11}= & g_{11}+\frac{\left[-2 M r^{3}+3\left(M^{2}+Q^{2}\right) r^{2}-6 M Q^{2} r+2 Q^{4}\right]}{4 r^{2}\left(r-2 M r+Q^{2}\right)^{2}} \theta^{2}+O\left(\theta^{4}\right) \\
\hat{g}_{22}= & g_{22}+\frac{1}{16}\left[1-\frac{15 M}{r}+\frac{26 Q^{2}}{r^{2}}+\frac{4\left(M r-Q^{2}\right)^{2}}{r^{2}\left(r^{2}-2 M r+Q^{2}\right)}\right] \theta^{2}+O\left(\theta^{4}\right) \\
\hat{g}_{33}= & g_{33}+\frac{1}{16}\left[\frac{4 r^{2}\left(M^{2}-M r\right)+8 Q^{2}\left(r^{2}-2 M r\right)+8 Q^{4}}{r^{2}\left(r^{2}-2 M r+Q^{2}\right)^{2}} \sin ^{2} \psi+\cos ^{2} \psi\right] \theta^{2}+ \\
& +O\left(\theta^{4}\right) .
\end{aligned}
$$

The above results match the previous neutral solution (27) found in Ref. 151, Contrary to what one would expect, the electromagnetic energy momentum tensor is kept in the ordinary form, namely no $\star$-deformation has been considered for the electromagnetic field. From a physical point of view, this fact appears unclear since the energy content of the electromagnetic field is expected to be intrinsically related to the fluctuation of the noncommutative manifold. Apart from these general comments, the major concern about the above metric is the still persistent inability to cure the curvature singularity, which, this time, affects both the gravitational field 
and the electromagnetic field, giving rise to even worse $1 / r^{6}$ terms. This is another malicious feature of the expansion of the $\star$-product in $\theta$, indeed a procedure without any outlet to reliable physical effects.

In the same way, another proposal for a noncommutative Reissner-Nordström solution exhibits a bad short distance behavior. By a mere substitution of the radial coordinate in terms of its noncommutative equivalent $r \rightarrow \hat{r}=\sum_{i}\left(\hat{x}^{i}\right)^{2}$, the following line element has been introduced in Ref. 237

$$
d s^{2}=\left(1-\frac{2 M}{\hat{r}}+\frac{Q^{2}}{\hat{r} \hat{r}}\right) d t^{2}-\frac{d \hat{r} d \hat{r}}{\left(1-\frac{2 M}{\hat{r}}+\frac{Q^{2}}{\hat{r} \hat{r}}\right)}-\hat{r} \hat{r}\left(d \psi^{2}+\sin ^{2} \psi d \phi^{2}\right) .
$$

As we have already seen for the Schwarzschild case 156|157, again the notation appears unclear: once $\hat{r}$ is written in terms of the matrix $\theta^{i j}$ and the conventional position operators $x^{i}$ and momenta $p_{i}, d s^{2}$ is still far from what we mean by line element. Despite this, allowing a sort of classical interpretation for the operators, an expansion over the noncommutative parameter has been performed, giving rise to

$$
g_{00} \simeq\left(1-\frac{2 M}{r}+\frac{Q^{2}}{r^{2}}\right)+\left(\frac{Q^{2}}{2 r^{4}}-\frac{M}{2 r^{3}}\right)\left[\vec{L} \vec{\theta}-\frac{1}{8}\left(p^{2} \theta^{2}-(\vec{p} \cdot \vec{\theta})\right)\right]+\mathcal{O}\left(\theta^{3}\right),
$$

where $L_{k}=\varepsilon_{i j k} x_{i} p_{j}$ and $\theta_{i j}=\frac{1}{2} \epsilon_{i j k} \theta_{k}$. Apart from the ambiguities, the proposed line element fails the main goal of any noncommutative geometry approach and exhibits, by the presence of the charge, an even worse $1 / r^{4}$ term, with an inconsistent spherical symmetry breaking.

\subsection{The noncommutative inspired Reissner-Nordström solution}

We are now ready to generalize the results already seen in Ref. 162 and study the noncommutative inspired charged black hole 238 , a geometry which is going to solve all of the inconsistencies of the above line element in a single stroke. We shall start from the new field, the Maxwell field $F^{\mu \nu}$, studying its behavior within the framework of noncommutative quasi coordinates. In agreement with what we have already seen for the linearized solution in Ref. 161, the Poisson equation governing the electrostatic potential exhibits an enlarged source term instead of the conventional Dirac $\delta$. Therefore, when a pointlike charge is considered in a noncommutative background, the effective current density is given by

$$
J^{\mu}(x)=\rho_{e l .}(x) \delta_{0}^{\mu}
$$

where, as expected, $\rho_{e l}$. has a Gaussian profile

$$
\rho_{e l .}=\frac{\mathcal{Q}}{(4 \pi \theta)^{3 / 2}} \exp \left(-\vec{x}^{2} / 4 \theta\right) .
$$

As a consequence the electric field $E(r)$ turns out to be an everywhere regular function

$$
E(r)=\frac{2 \mathcal{Q}}{\sqrt{\pi} r^{2}} \gamma\left(\frac{3}{2} ; \frac{r^{2}}{4 \theta}\right)
$$


giving rise to a nonsingular Maxwell field $F^{\mu \nu}=\delta^{0[\mu \mid} \delta^{r \mid \nu]} E(r)$. With the above ingredients, we are ready to consider the system describing quasi classically both the electromagnetic field and the gravitational field, a system which resembles the conventional Einstein-Maxwell system

$$
\begin{aligned}
& R^{\mu}{ }_{\nu}-\frac{1}{2} \delta^{\mu}{ }_{\nu} R=8 \pi\left(\left.T_{\nu}^{\mu}\right|_{\text {matt. }}+\left.T_{\nu}^{\mu}\right|_{e l .}\right) \\
& \frac{1}{\sqrt{-g}} \partial_{\mu}\left(\sqrt{-g} F^{\mu \nu}\right)=J^{\nu} .
\end{aligned}
$$

Here $\left.T_{\nu}^{\mu}\right|_{\text {matt. }}$ is the same as in the neutral case, describing the energy content of the matter, while $\left.T_{\nu}^{\mu}\right|_{e l}$., even if formally the usual one, takes into account, through the form of $F^{\mu \nu}$, the smearing effect to which the propagation of the electromagnetic field is subject when noncommutativity is considered. Thus, looking for a line element of the form $d s^{2}=-f(r) d t^{2}+f^{-1}(r) d r^{2}+r^{2} d \Omega^{2}$, one finds

$$
f(r)=1-\frac{4 \mathcal{M}}{r \sqrt{\pi}} \gamma\left(3 / 2, r^{2} / 4 \theta\right)+\frac{Q^{2}}{\pi r^{2}} F(r),
$$

where $\mathcal{M}$ is the "bare" mass parameter, while

$$
F(r) \equiv \gamma^{2}\left(1 / 2, r^{2} / 4 \theta\right)-\frac{r}{\sqrt{2 \theta}} \gamma\left(1 / 2, r^{2} / 2 \theta\right),
$$

and the charge $Q=\mathcal{Q}$ in natural unit: 0 . To have a deeper understanding of the above solution, it is convenient to introduce the total mass-energy of the electrogravitational system

$$
M=\oint_{\Sigma} d \sigma^{\mu}\left(\left.T_{\mu}^{0}\right|_{\text {matt. }}+\left.T_{\mu}^{0}\right|_{e l .}\right)
$$

where $\Sigma$ is a $t=$ const., closed three-surface. In terms of $M$ the solution reads

$$
f(r)=1-\frac{4 M}{\sqrt{\pi} r} \gamma\left(3 / 2, r^{2} / 4 \theta\right)+\frac{Q^{2}}{\pi r^{2}}\left[F(r)+\sqrt{\frac{2}{\theta}} r \gamma\left(3 / 2, r^{2} / 4 \theta\right)\right]
$$

which exhibits the expected asymptotic behavior, reproducing the ReissnerNordström geometry at large distances.

Our analysis proceeds with the study of the horizon equation $f=0$, that can be efficiently visualized in terms of the plot in Fig. 9. We can see that in analogy with the noncommutative Schwarzschild case, there are three possible cases, this time depending on the values of both $M$ and $Q$ : therefore the line element (84) describes either a two-horizon charged black hole or an extremal single horizon charged black hole or a charged gravitational object without any horizon. A key aspect of the solution is given by the fact that in all such cases, the conventional curvature singularity at the origin is smeared out by the noncommutative fluctuations of the spacetime manifold. Indeed the short distance behavior of the metric is of the deSitter type

$$
g_{00}=1-\frac{\mathcal{M}}{3 \sqrt{\pi} \theta^{3 / 2}} r^{2}+O\left(r^{4}\right)
$$

${ }^{\circ}$ Otherwise one has $Q=\mathcal{Q} G^{1 / 2} / c^{2}$ 


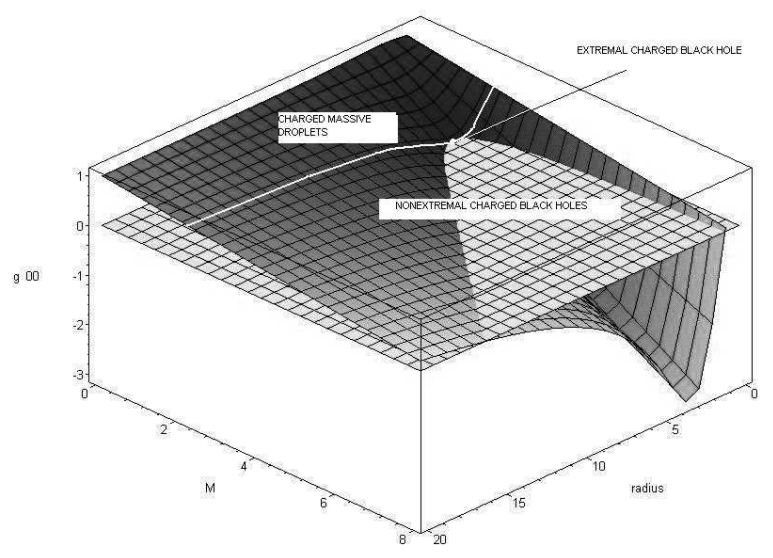

Fig. 9. The noncommutative Reissner-Nordström solution. The function $f$ is plotted versus $M$ and $r$, for a charge, $Q=1$ in $\sqrt{\theta}$ units. The intersection of the $f=0$ plane (light-grey) with $f=f(r, M)$ surface (dark-grey) gives the "horizon curve" whose minimum (white-dot) gives the extremal black hole. The portion of the surface below the plane $f=0$ represents the spacelike region between the inner and outer horizons.

whose effective cosmological constant $\Lambda_{\text {eff. }}=\mathcal{M} / \sqrt{\pi} \theta^{3 / 2}$ is governed only by the "bare" mass because the electric field contributes with subleading $O\left(r^{4}\right)$ terms, due to its linear behavior at short distance, i.e. $E(r) \sim Q r / 6 \sqrt{\pi} \theta^{3 / 2}$.

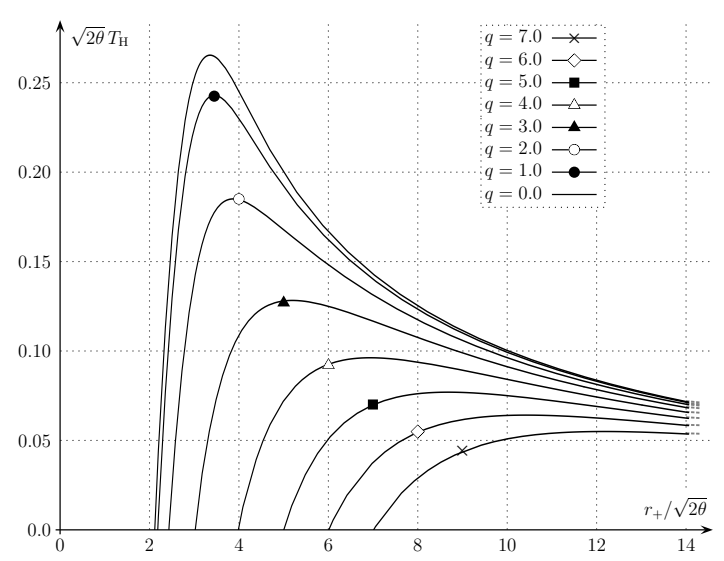

Fig. 10. The noncommutative Reissner-Nordström solution. The Hawking temperature $T_{H} \times \sqrt{2 \theta}$ as a function of $r_{+} / \sqrt{2 \theta}$, for different values of $Q$ in $\sqrt{\theta}$ units. The temperature drops to zero even in the case $Q=0$ as a result of coordinate uncertainty. The peak temperature drops with increasing Q. 


\subsubsection{Black hole temperature, Hawking and Schwinger mechanisms}

The regularity of the geometry has its thermodynamic equivalent in the finiteness of the black hole temperature, which is given by

$$
\begin{aligned}
4 \pi T_{H} & =\frac{1}{r_{+}}\left[1-\frac{r_{+}^{3} \exp \left(-r_{+}^{2} / 4 \theta\right)}{4 \theta^{3 / 2} \gamma\left(3 / 2, r_{+}^{2} / 4 \theta\right)}\right]+ \\
& -\frac{4 Q^{2}}{\pi r_{+}^{3}}\left[\gamma^{2}\left(3 / 2, r_{+}^{2} / 4 \theta\right)+\frac{r_{+}^{3} \exp \left(-r_{+}^{2} / 4 \theta\right)}{16 \theta^{3 / 2} \gamma\left(3 / 2, r_{+}^{2} / 4 \theta\right)} F\left(r_{+}\right)\right]
\end{aligned}
$$

where $r_{+}$is the outer horizon. The above formula contains the expression for the temperature already seen in the neutral Schwarzschild case, with an additional term due to the presence of the charge and responsible for a global lowering of the profile of $T_{H}$. At this point, one would be tempted to say that the final configuration of the evaporation is an extremal black hole, with a nonzero residual charge, a radius and mass larger than $3.02 \sqrt{\theta}$ and $1.90 \sqrt{\theta}$, the values, which we obtained for the neutral remnant. Against this scenario, there is the fact that, conventionally, the electric charge of a black hole is negligible, since the neutralization phase occurs in very short times 2131239 . Furthermore, pairs of charges can be produced quantum mechanically in the black hole's surroundings, reducing even faster the black hole electric charge to zero. The rate of production of pairs of particles in now ruled not only by the Hawking emission, but also via the Schwinger mechanism $\frac{240}{20}$ for the presence of the black hole electric field. Indeed if the electrostatic potential energy on the black hole outer horizon $r_{+}$is sufficiently high, at least locally it is possible to have a uniform electric field exceeding the threshold electric field $E_{t h}=\pi m_{e}^{2} / e$ and priming the pair production $241-254$. As a result, we expect that the charged black hole balding phase will occur in a very short time, leading to a Schwarzschild phase and finally to a SCRAM phase terminating with the formation of a zero temperature stable black hole relic, which we already described by means of the noncommutative inspired Schwarzschild solution. For this reason, in Fig. 10, the region of the plot near to the intersections with the $r_{+}$-axis, does not correspond to a physically realizable situation, since charged remnants do not develop. As a consequence, the black hole back reaction due to the Hawking emission mechanism, is still negligible: indeed the balding phase is so quick that, at a given $r_{+}$the black hole is always colder and heavier than its neutral equivalent. In support of the above reasoning, it is sufficient to require that the electric field at $r_{+}$exceeds the threshold field, namely $E\left(r_{+}\right)>E_{t h}=\pi m_{e}^{2} / e$. Writing the black hole total charge as $Z e$, i.e. an integer multiple of the elementary charge, one finds that at any possible value for $r_{+}$, just a single electron charge, namely having $Z=1$, is sufficient to have pair creation in the black hole surroundings. Indeed the threshold field, once written in terms of $\theta$ units, is $E_{t h} \sim m_{e}^{2} \theta<<1$ and therefore the black hole electric field is so strong that the Schwinger mechanism can occur even far away from the black hole outer horizon. In view of this, it is useful to introduce the concept of dyadosphere, representing the spherical region where the electric field reaches the threshold value 
and produces pairs: then, in our case, the dyadosphere radius $r_{d s}$ can be determined by

$$
\frac{r_{d s}^{2}}{\gamma\left(\frac{3}{2} ; \frac{r_{d s}^{2}}{4 \theta}\right)}=\frac{2}{\pi^{3 / 2}} \frac{Z e^{2}}{m_{e}^{2}}
$$

As a first approximation, we find

$$
r_{d s}^{2} \simeq \frac{2 Z e^{2}}{\pi^{3 / 2} m_{e}^{2}} \gamma\left(\frac{3}{2} ; \frac{Z e^{2}}{4 \pi \theta m_{e}^{2}}\right)
$$

which lets us estimate the dyadosphere radius as being comparable with the electron Compton wave length $r_{d s} \sim 1 / m_{e} \gg \sqrt{\theta}$. From the large extension of the dyadosphere with respect to the noncommutative scale $\sqrt{\theta}$, we conclude that the black hole described by the line element (84) is extremely unstable under Schwinger pair production, a mechanism which dominates the early life of the black hole until a neutral phase is reached. An important note regards the hot debate in the literature about the occurrence of a dyadosphere for astrophysical black holes 255 257. Without entering this debate, it is necessary to show that the criticisms against dyadosphere formation do not apply to objects like the noncommutative black holes, which are governed by the scale $\sqrt{\theta}$ : indeed we find that in our case the condition for the existence of the dyadosphere

$$
\frac{E}{E_{c}}=\frac{e \mathcal{Q}}{r^{2} m_{e}^{2}} \frac{2}{\sqrt{\pi}} \gamma\left(\frac{3}{2} ; \frac{r^{2}}{4 \theta}\right) \ll \frac{m_{p} M G}{r^{2} m_{e}^{2}} \sim\left(\frac{m_{p}}{m_{e}}\right) \frac{M}{m_{e}} \sim 4 \times 10^{9}
$$

is always met, where $m_{p}$ is the proton mass. Thus we can conclude that the electric field can reach the threshold value without the electrostatic repulsion overcoming the gravitational attraction among hadronic charged matter. For the above reason, it is legitimate to follow the lines suggested in Refs. 252, 254, to have an estimate of the discharge time via the Schwinger mechanism. To this purpose it is worthwhile to introduce the surface charge density $\sigma(r)$, in order to divide the dyadosphere into thin spherical shells of thickness $1 / 2 m_{e}$. Calculating the number of pairs produced, per second inside such a spherical shell because of the presence of the electric field $E(r)=4 \pi \sigma(r)$, one finds that the discharge time is

$$
\Delta \tau=\frac{\theta m_{e}}{\alpha_{e m}}\left(\frac{2 \pi}{m_{e} c \sqrt{\theta}}\right)^{2} \frac{s-1}{s^{2}} \exp \left(\frac{\pi}{s}\right)
$$

where $\alpha_{e m}=1 / 137$ is the fine structure constant and $s=\sigma / \sigma_{c}$, with the critical surface density $\sigma_{c}=m_{e}^{2} / 4 \pi e$ being obtained when $E=E_{t h}$. An upper bound on $\Delta \tau$ can be obtained for $s \approx 1$, i.e. $\sigma \approx \sigma_{c}$, to obtain $\Delta \tau \leq 1.76 \times 10^{-19}$ s. This result confirms that the black hole tends to discharge in a very short time, reaching a Schwarzschild phase. 


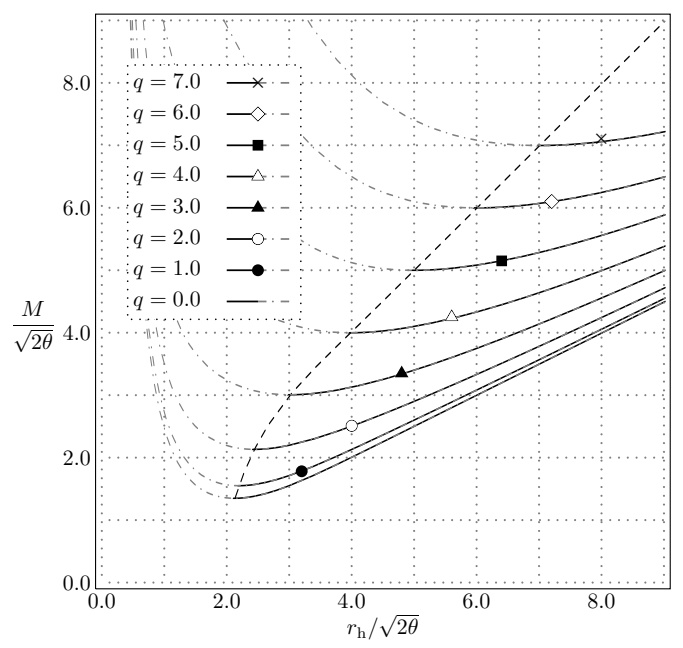

Fig. 11. The noncommutative Reissner-Nordström solution The total mass energy $M / \sqrt{2 \theta}$ is plotted as a function of $r_{+} / \sqrt{2 \theta}$ for different $q \equiv Q / \sqrt{2 \theta}$. At the very beginning, the system is described by $Q$ and $r_{+}$, then it starts evolving towards its ground state, i.e. it tends to minimize its total mass-energy both by sliding down the $q$-curve and by jumping from higher to lower $q$-curves. The Schwarzschild black-hole is given by the lowest curve, $q=0$, which is no longer a straight line indicating that there can be two horizons for $M>1.90 \sqrt{\theta}$. The dashed curve intersects the minima of the $q$-curves representing extremal black holes and deviating from the conventional linear behavior $M=r_{+} / 2$ during the SCRAM phase because of the presence of the residual nonzero mass of the remnant.

\subsubsection{Charged black hole entropy}

As final note of this section, we analyze the entropy of this charged black hole, which has a further term with respect to the neutral solution. Starting from Ref. 213

$$
d M=T_{H} d S_{H}+\frac{\partial M}{\partial Q} d Q
$$

where the mass is a function of both $r_{+}$and $Q$, namely $M=M\left(r_{+}, Q\right)$ through the horizon equation. As a consequence, we have

$$
d M=\frac{\partial M}{\partial r_{+}} d r_{+}+\frac{\partial M}{\partial Q} d Q
$$

Comparing (91) and (92) one finds the following useful relation for the entropy

$$
d S_{H}=\frac{1}{T_{H}} \frac{\partial M}{\partial r_{+}} \cdot d r_{+}
$$

Therefore we have to calculate the mass derivative with respect to $r_{+}$and determine the expression for $d S_{H}$, that once integrated from the extremal horizon $r_{0}$ up to a 
generic external horizon $r_{+}$gives

$$
\begin{aligned}
\Delta S_{H} & =\frac{\pi^{3 / 2}}{2}\left[\frac{r_{+}^{2}}{\gamma\left(3 / 2, r_{+}^{2} / 4 \theta\right)}-\frac{r_{e}^{2}}{\gamma\left(3 / 2, r_{e}^{2} / 4 \theta\right)}\right]+ \\
& +\frac{\pi^{3 / 2}}{2} \int_{r_{e}}^{r_{+}} d u u^{2} \frac{\gamma^{\prime}\left(3 / 2, u^{2} / 4 \theta\right)}{\gamma\left(3 / 2, u^{2} / 4 \theta\right)^{2}} .
\end{aligned}
$$

Even if both $r_{0}$ and $r_{+}$do depend on the charge $Q$, the above expression can be rewritten as

$$
\Delta S_{H}=\frac{1}{4 G_{\theta}(r)}\left(A_{+}-A_{e}\right)+\delta S_{H}
$$

which reproduces the celebrated relation $S_{H}=A_{H} / 4$ in the same way as we have already seen for the neutral solution in (59), after the introduction of the effective fundamental scale $G_{\theta}(r)$. Again it can be shown that the correcting term $\delta S_{H}$ in (95) is always exponentially small. Thus we can conclude that the area law is maintained up to exponentially small corrections 200 .

\section{The Extradimensional Scenario}

All of the noncommutative black hole solutions presented until now, both for the neutral and charged cases, have been proposed in the literature as possible candidates to describe the final phase of the evaporation and the onset of quantum gravity. Even if the regularity of a solution at the origin can be assumed as a mandatory requirement on the theoretical side, we do not yet have a way to discriminate the correctness of a model on an experimental basis. To this purpose, a possible way out comes from $\mathrm{TeV}$-scale Quantum Gravity and the conjecture of production of black holes at the CERN Large Hadron Collider within a few months. Therefore, it is imperative to analyze the extensions of the proposed models to the case of large spatial extradimensions, assuming a unique mass scale $M_{\theta} \approx M_{*} \sim 1 \mathrm{TeV}$, in order to study the new physics coming from noncommutativity. More in detail, even if the noncommutative mass scale $M_{\theta}$ is directly correlated to $\sqrt{\theta}$, it is sufficient to assume only that $\sqrt{\theta} \approx 1 / M_{\theta}$, without specifying the exact relationship. Indeed, depending on the models $258-285$, we could find that $M_{\theta} \sim 1-10 \mathrm{TeV}$. On the other hand, independently of noncommutativity, there is also a huge amount of work about the possibility of observing mini black holes by detecting the products of the Hawking/Schwinger mechanism or even revealing the presence of any black hole remnant by the study of visible and missing momenta of the hadronic fragments after the collision 286 305. All such kinds of analysis are performed in terms of semiclassical arguments and the conventional Schwarzschild geometry. For this reason, on similar grounds, the fate of any evaporating black hole can only be described by means of speculative scenarios, since no reliable prediction can be made if the black hole mass $M \sim M_{*}$. 


\subsubsection{The noncommutative higher dimensional Schwarzschild solution}

In such a scheme it is natural to proceed along the lines proposed in Ref. 306 , in order to combine, for the first time, noncommutative phenomenology and the properties of TeV-scale mini Schwarzschild black holes in order to overcome the basic limitations of semiclassical approaches. In the spirit of the four dimensional noncommutative inspired solutions $\frac{162 \mid 238}{}$, we will determine a smearing of matter distributions on length scales of order $\sqrt{\theta}$, while, as usual, the geometry sector is formally left unchanged. Furthermore, to efficiently employ the condition of spherically symmetry, we need that both the black hole horizon size and the noncommutative length scale $\sqrt{\theta}$ be smaller than $R$, the size of each of the $n$ extradimensions. For later convenience, it is worthwhile to introduce $d=3+n$, the total number of spatial dimension, i.e. $D=d+1$. Therefore the noncommutative equivalent of the higher dimensional Einstein equation comes from the $D$ dimensional Einstein Hilbert action

$$
S=\frac{M_{*}^{d-1}}{2} \int \sqrt{-g} \mathcal{R}
$$

with $\mathcal{R}$ being the Ricci scalar in terms of noncommutative quasi coordinates, while the source term for a massive object is determined by $\sqrt{\theta}$ in terms of a static spherically symmetric Gaussian matter distribution

$$
\rho(r)=\frac{M}{(4 \pi \theta)^{d / 2}} \exp \left(-r^{2} / 4 \theta\right) .
$$

For the above conditions, the metric is assumed to be of the spherically symmetric form

$$
d s_{(d+1)}^{2}=-f(r) d t^{2}+f^{-1}(r) d r^{2}+r^{2} d \Omega_{d-1}^{2}
$$

with a further demand that $f(r) \rightarrow 1$ as $r \rightarrow \infty$, while $\Omega_{d-1}^{2}$ can be simply described in terms of $d-1$ angles, $\phi_{i}$ where $i=1, \ldots, n+2$. In analogy to what we have seen in the four dimensional case, two component of the energy momentum tensor $T^{M N}$ are already determined, $T_{0}^{0}=T_{r}^{r}=-\rho$, while the remaining $d-1$ components, which are identical from the condition of spherical symmetry, can be obtained by requiring the covariant conservation of $T^{M N}$, namely $T^{M N}{ }_{; N}=0$. As a result, we find

$$
T_{i}^{i}=-\rho-\frac{r}{d-1} \partial_{r} \rho
$$

for all $i=1, \ldots, d-1$ (without summation), reproducing the the four dimensional energy momentum tensor in the limit when $d \rightarrow 3$, i.e. $n \rightarrow 0$. Therefore $T^{M N}$ describes a $d$-dimensional anisotropic fluid and provides the source term of the resulting Einstein equation in the presence of noncommutative smearing effects

$$
R_{M}^{N}=\frac{1}{M_{*}^{d-1}}\left(8 \pi T_{M}^{N}-\frac{8 \pi}{d-1} \delta_{M}^{N} T\right)
$$


where $T$ is the trace of the energy momentum tensor, $T=T_{M}^{M}$. Plugging the line element (98) into the above equation, one finds a solution in terms of $f(r)$

$$
f(r)=1-\frac{1}{M_{*}^{d-1}} \frac{2 M}{r^{d-2} \Gamma(d / 2)} \gamma\left(\frac{d}{2}, \frac{r^{2}}{4 \theta}\right)
$$

where

$$
\gamma\left(d / 2, r^{2} / 4 \theta\right) \equiv \int_{0}^{r^{2} / 4 \theta} \frac{d t}{t} t^{d / 2} e^{-t}
$$

and with

$$
\begin{gathered}
\Gamma(d / 2)=\left(\frac{d}{2}-1\right) ! \quad \text { d even } \\
\Gamma(d / 2)=\sqrt{\pi} \frac{(d-2) ! !}{2^{(d-1) / 2}} \quad d \text { odd } .
\end{gathered}
$$

\subsubsection{Curvature, horizons and remnants}

The above result reproduces the four dimensional line element when $d \rightarrow 3$ i.e. $n \rightarrow 0$, since $G=M_{*}^{-2}$, with $M_{*}=M_{P}$. Also the commutative limit works, because the usual $D$-dimensional Schwarzschild solution is reproduced when $\sqrt{\theta} / r \rightarrow$ d . On the other hand the behavior of the manifold near to the origin is given by

$$
f(r)=1-\frac{1}{d M_{*}^{d-1}} \frac{4 M}{2^{d-1} \pi^{(d-2) / 2} \theta^{d / 2}} r^{2}+0\left(r^{4}\right)
$$

being of deSitter type, as expected. Again, the $D$-dimensional curvature singularity has been dragged out by the noncommutative fluctuations.

Table 1. Remnant masses and radii for different values of $d$, keeping $M_{*} \sim 1 / \sqrt{\theta}$

\begin{tabular}{ccccccccc}
\hline $\mathrm{d}$ & 3 & 4 & 5 & 6 & 7 & 8 & 9 & 10 \\
\hline$M_{0}(\mathrm{TeV})$ & $2.3 \times 10^{16}$ & 6.7 & 24 & 94 & $3.8 \times 10^{2}$ & $1.6 \times 10^{3}$ & $7.3 \times 10^{3}$ & $3.4 \times 10^{4}$ \\
\hline$r_{0}\left(10^{-4} \mathrm{fm}\right)$ & $4.88 \times 10^{-16}$ & 5.29 & 4.95 & 4.75 & 4.62 & 4.52 & 4.46 & 4.40 \\
\hline
\end{tabular}

From the horizon equation $f(r)=0$, one again obtains three possibilities depending on the value of the mass $M$, namely two horizons, one single degenerate horizon and no horizon. The plot in Fig. 12 shows the behavior of the outer horizon radius $r_{+}$, which decreases as $d$ increases for a given mass $M$. The minimum of each

p The commutative limit of the above line element differs from the usual form of the higher dimensional Schwarzschild solution in most of the literature 307 ,

$$
f(r)=1-\frac{1}{M_{*}^{d-1}} \frac{4 \Gamma(d / 2)}{(d-1) \pi^{(d-2) / 2}} \frac{2 M}{r^{d-2}}
$$

because in the present case the extradimensions were not compactified and therefore the constants were not matched to the four dimensional ones. Anyway, the additional factors, being of order one, can be reabsorbed in the definition of the fundamental scale $M_{*}$. 


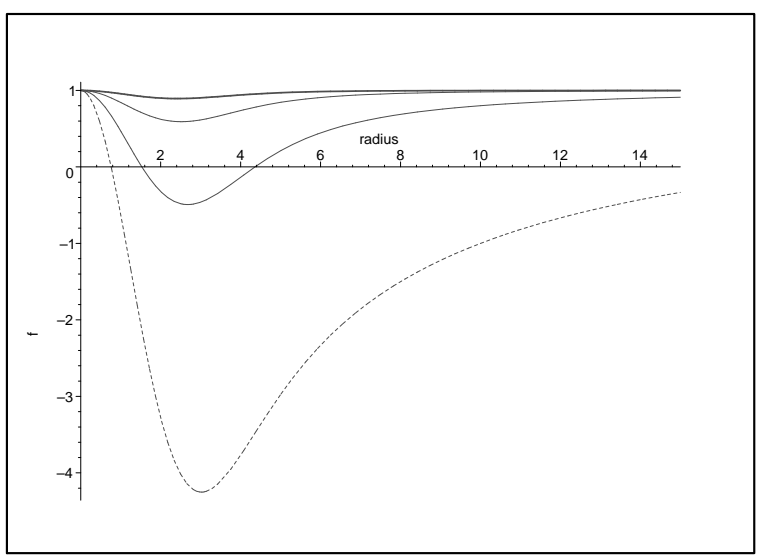

Fig. 12. The noncommutative extradimensional Schwarzschild solution. The function $f$ is poltted versus $r / \sqrt{\theta}$, for $M=10 M_{*}$. We can observe that the curves rise with $d$ and the outer horizon $r_{+}$ decreases until $d=4$. For $d \geq 5$ no black hole can be formed: the mass $M$ is so light that cannot provide a significant gravitational disturbance, since $f \simeq 1$.

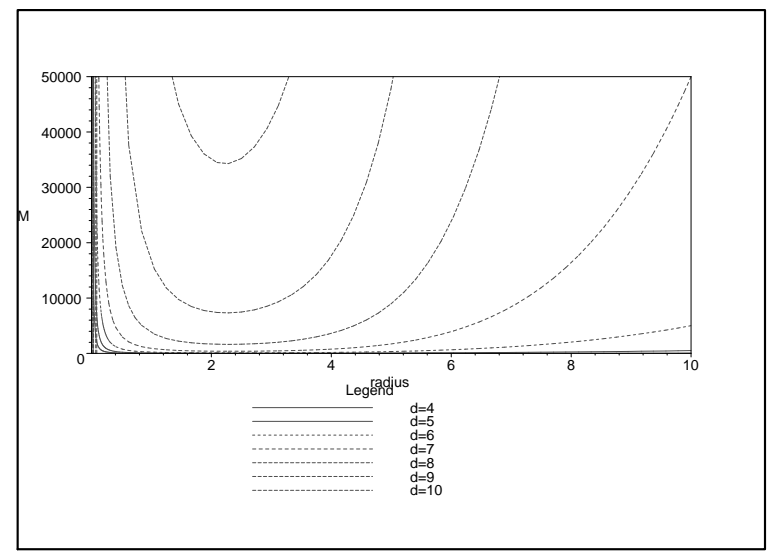

Fig. 13. The noncommutative extradimensional Schwarzschild solution. The mass $M$ as a function of $r_{+}$in $\sqrt{\theta}$ units for different values of $d$. The resulting minima of these curves provide the remnant masses, which increase with $d$, while their radii decrease.

curve also provides the value of the extremal radius $r_{0}$ which again decreases with $d$. Conversely from Fig. 13, we obtain that the remnant masses increase with $d$, even if their radii decrease. The values of mass and radius for the remnant are summarized in Table 1. Since remnant radii are $\sim 4-5 \times 10^{-4} \mathrm{fm}$, i.e. smaller than the size of extradimensions, these remnants can be considered, to a good approximation, as totally submerged in a $D$ dimensional isotropic spacetime, for any $d=3-10$. As further consequence, estimating the black hole production cross section as the area of the event horizon $\sigma \sim \pi r_{+}^{2}$, we find, for every $d$, an encouraging lower bound $\sigma \gtrsim 10 \mathrm{nb}$, almost two orders of magnitude larger than the conventional values. On 
the other hand, for $d \geq 6$ the remnant is too heavy to be produced at the LHC and could be only detected in Ultra-High-Energy cosmic rays $\$ 33 \mid 308$.

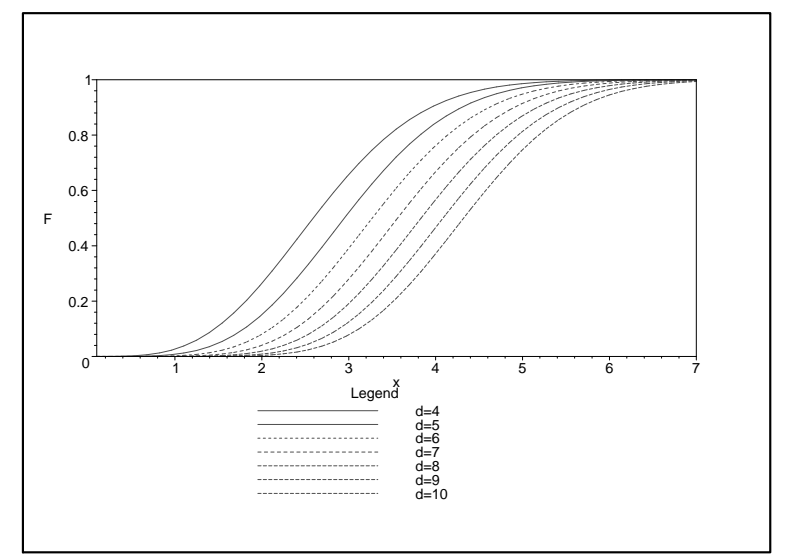

Fig. 14. The noncommutative extradimensional Schwarzschild solution. The function $G_{d}$ vs $z \equiv$ $x / y$ for different value of $d$. The noncommutative effects are relevant only for $z \lesssim 7$, namely when the horizon size is within the noncommutative scale. The function $G_{d}$ is the dimensionless effective Newton constant and realizes the "asymptotically safe" gravity conjecture at short distances for any $d$. Furthermore, we find that $G_{d}$ is equivalent to $x_{\theta} / x$, representing the ratio between the horizon value $x$, solution of the Eq. (108) and the conventional result $(2 m)^{1 /(d-2)}$.

A more quantitative analysis can be made by the introduction of dimensionless quantities $m \equiv M / M_{*}, x \equiv r_{+} M_{*}$ and $y \equiv \sqrt{\theta} M_{*}$ in order to write the horizon equation in terms of

$$
x^{d-2}=2 m G_{d}(z)
$$

where

$$
G_{d}(z)=\frac{1}{\Gamma(d / 2)} \gamma\left(d / 2, z^{2} / 4\right)
$$

is the dimensionless effective Newton constant, with $z \equiv x / y$. Fig. 14 shows how gravity becomes weaker and weaker at short distances because of the presence of noncommutative fluctuations of spacetime. Indeed the noncommutative smearing realizes the "asymptotically safe" gravity conjecture, eliminating the curvature singularity without invoking any RG arguments203/204. When $d$ is even, the incomplete gamma function can be written in explicit form, namely $G_{4}=$ $1-e^{-p}(1+p), G_{6}=1-e^{-p}\left(1+p+p^{2} / 2\right), G_{8}=1-e^{-p}\left(1+p+p^{2} / 2+p^{3} / 6\right), \ldots$, $G_{d}=1-e^{-p}\left(1+p+p^{2} / 2+\ldots+p^{d-5} /(d-5)\right)$, where $p \equiv z^{2} / 4$. From Eq. (108), one can obtain that the behavior of $m$ at infinity and near to the origin is $\sim x^{d-2}$ and $\sim x^{-2}$ respectively, implying the existence of a minimum value of $m$ for some intermediate value of $x$, in agreement with Fig. 13. Furthermore, in the commutative 
limit, i.e. $y, \theta \rightarrow 0$, one can reproduce the usual result

$$
x=(2 m)^{1 /(d-2)}
$$

a relationship that no longer occurs in the noncommutative case. Again from Fig.14, we find that, for all $d$, the noncommutative value of $x$ deviates from the usual value $(2 m)^{1 /(d-2)}$ in a region around the origin, because only there is the noncommutative scale comparable with the horizon size. As a consequence, the existence of a minimum for the mass $m$ implies also a minimum for the horizon radius $x$ and a physical mass threshold below which black holes do not form.

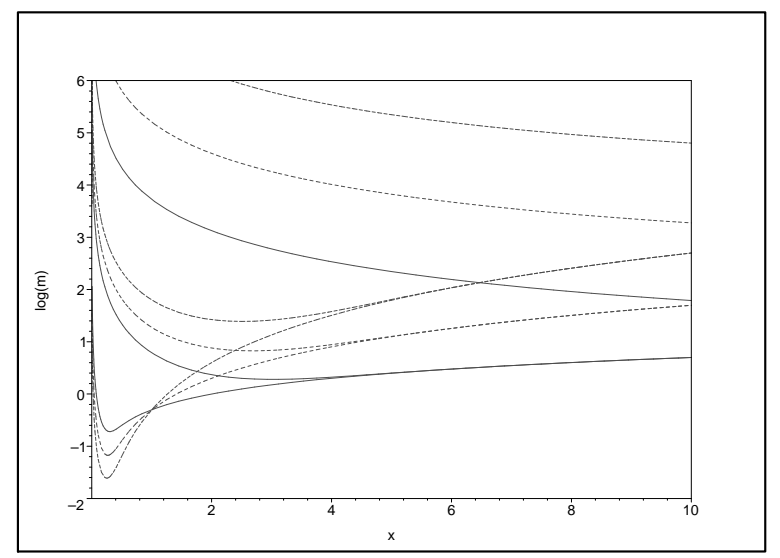

Fig. 15. The noncommutative extradimensional Schwarzschild solution. The black hole mass, $\log _{10}(m)$, as a function of $x$ for different values of $d=3$ (solid), $d=4$ (dotted) and $d=5$ (dashed). The upper (middle, lower) set of curves correspond to $y=10(1,0.1)$.

The analysis in Table1 1 shows that black holes are too massive to be produced at LHC for large $d$, assuming that $\sqrt{\theta} M_{*}=y=1$. More generally we can also calculate $m$ as a function of $x$, varying $y$, the parameter governing the noncommutative scale. From Fig. 15, one finds that as $y$ increases for fixed $d$ so does $m$ except where $x$ is large and we are probing the commutative regime. Therefore, we need small values of $y$, i.e. $0.05 \lesssim y \lesssim 0.2$, to obtain a range of masses accessible to the LHC, namely $1 \lesssim m \lesssim 10$, assuming $M_{*} \sim 1 \mathrm{TeV}$. On the other hand, having low values for $y$ leads to the commutative regime, in which both the minimum value of the mass, $m_{\min }$ and the horizon radius where the minimum mass occurs, $x_{\min }$ are algebraically zero. For this reason, it is worthwhile to assume that $m_{\min } \simeq \operatorname{Max}\left(1, m_{\min }^{a}\right)$, where $m_{\min }^{a}$ is the algebraic minimum value calculated from the equation $\partial m / \partial x=0$, i.e.

$$
G_{d}(p)-\frac{2 p^{d / 2} e^{-p}}{(d-2) \Gamma(d / 2)}=0
$$

In the same way, a small value of $y$ implies a lowering of the value of $x$, with consequent reduction of black hole production cross section, whose minimum $\sigma_{\min } \simeq$ 
$\pi x_{\min }^{2} / M_{*}^{2}$ is quite small even if not vanishing. For $y=0.05$, one finds $\sigma_{\min } \approx 20$ $\mathrm{pb}$, one order of magnitude below the value expected, on qualitative arguments, for a Schwarzschild black hole in $\mathrm{TeV}$ gravity. For energies far above the black hole production threshold, the cross section significantly increases according to $\sigma \sim$ $m^{2 /(d-2)}$, as in the commutative theory.

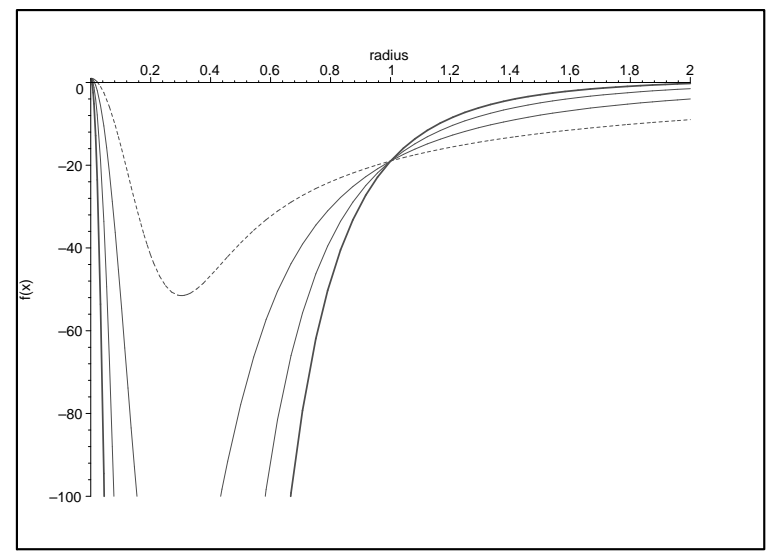

Fig. 16. The noncommutative extradimensional Schwarzschild solution. The function $f(x)$ versus $x=r M_{*}$, for $m=10$ and $y=0.1$, namely both the black hole mass and the noncommutative scale are heavier that the fundamental scale. Therefore nonextremal black holes form in a regime which resembles the commutative case. The dotted curve corresponds to $d=3$, while from top to bottom on the left hand side of the figure the solid curves are for $d=4$ to 6 . We can observe that the outer horizon radius decreases with $d$, while the curvature near to the origin, even if finite, increases with the smallness of $y$ (contraction of the deSitter core). The position of the minima of the above curves provides the radius of the extremal black hole $x_{0}$; for $d=3$ one finds $x_{3} \approx 3.0 y$ as expected.

Also the analysis of the horizon equation is somehow incomplete: the Fig. 12 provides the profile of $f(r)$ for $y=1$ only. Even if qualitatively we do expect a scenario with two (one or no) horizons analogous to the case for $y$ fixed, it is worthwhile to consider the general case $f=f(x, y, m, d)$ to get quantitatively reliable results. Indeed the parameter $y$, setting the noncommutative scale, has a crucial role: it determines whether or not it is possible to experience the noncommutative fluctuations of the manifold at the scale $M_{*}$. Phenomenologically we expect that $y \sim 0.1-10$, corresponding to the case of a manifold almost in a commutative regime, since the noncommutative scale too large $(y \sim 0.1)$; to the case for which the spacetime is dominated by the relevant noncommutative fluctuations, weakly resembling to the concept of classical manifold $(y \sim 10)$. Furthermore the parameter $y$ controls the extension of the portion of the manifold subject to the noncommutative regime: roughly speaking we can estimate this region from (106), calculating the extension, $L$, of the deSitter core around the origin even if noncommutativity 


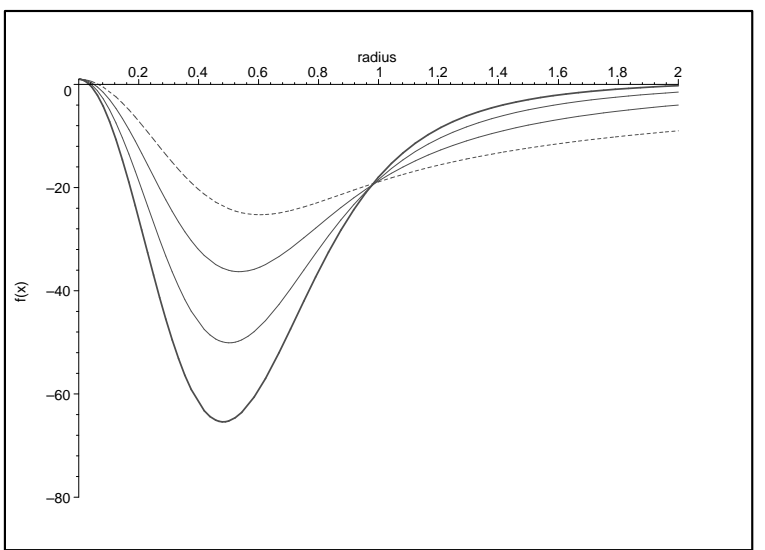

Fig. 17. The noncommutative extradimensional Schwarzschild solution. The function $f(x)$ versus $x=r M_{*}$, for $m=10$ and $y=0.2$. Each curve rises with respect to the case $y=0.1$, since the curvature at the origin decreases. Nonextremal black holes form with outer horizons that still occur at commutative values $(2 m)^{1 /(d-2)}$. The dotted curve corresponds to $d=3$, while from top to bottom on the left hand side of the figure the solid curves are for $d=4$ to 6 . We can observe that in agrement with Fig. 13 the extremal radius $x_{0}$ shrinks as $d$ increases.

can still be nonnegligible even beyond it

$$
L \sim y^{d / 2} m^{-1 / 2} \frac{1}{M_{*}} .
$$

Thus, we find a contraction of this core when $y<1$ for an increase of $d$ and viceversa an expansion when $y>1$. A fact related to the extension of the deSitter core is the value of the curvature at the origin: large curvatures implies smaller cores and viceversa. We can see this, looking at the Ricci scalar

$$
\mathcal{R} \sim \frac{m}{y^{d}} \exp \left(-x^{2} / 4 y^{2}\right)
$$

which, at a given mass $m$, essentially depends only on $y$ near the origin, namely $\mathcal{R} \sim y^{-d}$. The parameter $y$ also controls the extremal black hole. To this purpose, we find that the extremal radius $x_{\min }$ linearly depends on $y$

$$
x_{\min }(d)=z_{0}(d) y
$$

where $z_{0}(d)$ is the root of the equation (110), corresponding to the extremal radius for $y=1$. On the other hand, from (107) one finds that the extremal mass is

$$
m_{\min }=\frac{1}{2} G_{d}^{-1}\left(z_{0}\right) z_{0}^{d-2} y^{d-2} .
$$

Therefore the product $z_{0} y$ determines the kind of growth of $m_{\min }$ with $d$.

With the above ingredients, we can analyze Figs.16] 26, which deal with function $f(x)$ for $3<d<6$ with scale parameters $1 \lesssim m \lesssim 10$ and $0.1 \lesssim y \lesssim 10$ and exhibit some common features. For instance, the outer horizon radius generally decreases with $d$ because the mass $m$ turns out to be further smeared along the additional 


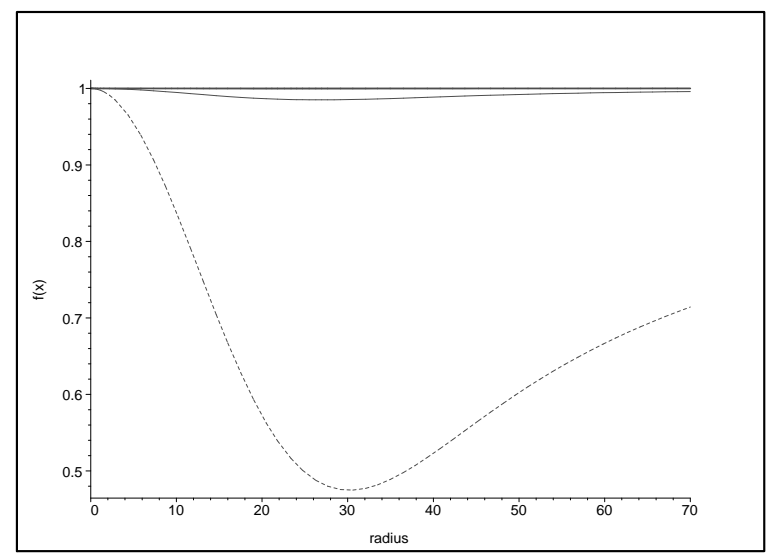

Fig. 18. The noncommutative extradimensional Schwarzschild solution. The function $f(x)$ versus $x=r M_{*}$, for $m=10$ and $y=10$. A larger $y$ implies an extension of the region where noncommutative fluctuations can be probed at the scale $M_{*}$, with a consequent wider smearing of the mass $m$ and a lowering of the manifold curvature. The smearing increases with $d$, because the mass $M$ is distributed within a larger spacetime, leading to $f \simeq 1$ for $d \geq 4$ : in other words since $y>1$ we assist an expansion of the deSitter core, which confines all of the mass $M$ within itself for $d \geq 4$. Therefore the noncommutative effects are so important that no black hole can form even for $d=3$.

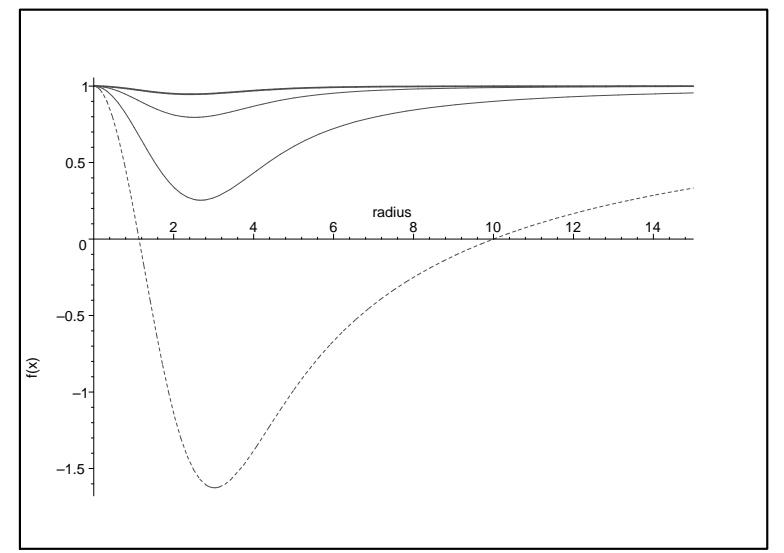

Fig. 19. The noncommutative extradimensional Schwarzschild solution. The function $f(x)$ versus $x=r M_{*}$, for $m=5$ and $y=1$. This borderline case occurs for $y=1$, where the smearing of the mass $m$ is relevant only in the presence of extradimensions. Indeed black holes do not develop for $d \geq 4$.

dimensions. Then, a large value of $y$, i.e. $y>1$, leads to weakly curved manifolds, because the smearing of the mass $m$ is so strong that it can disturb little the spacetime geometry. An alternative explanation is that the deSitter core is large enough to confine almost all of the mass inside itself, preventing the formation of a Schwarzschild region and consequent development of horizons. There are also 


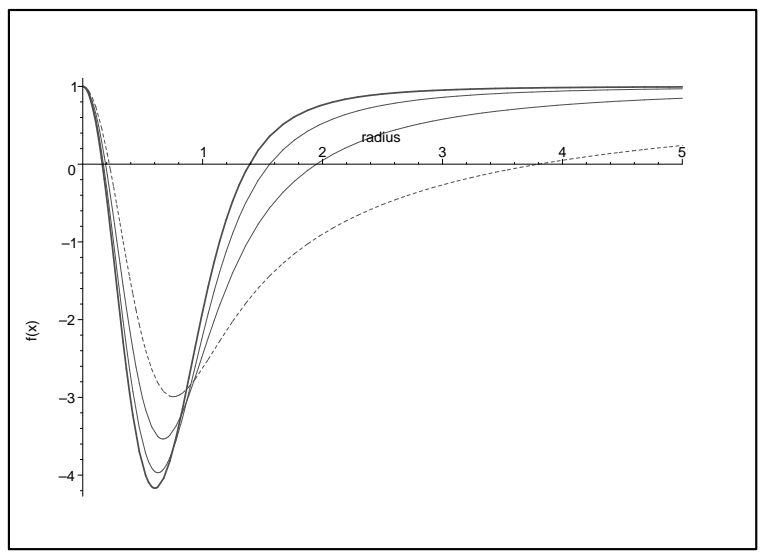

Fig. 20. The noncommutative extradimensional Schwarzschild solution. The function $f(x)$ versus $x=r M_{*}$, for $m=1.9$ and $y=0.25$. The mass parameter has the value equivalent to the extremal case for $d=3$, but the reduced value of $y$ implies a mass energy rather concentrated near the origin with a consequent formation of two horizons. The dotted curve corresponds to $d=3$, while from top to bottom on the left hand side of the figure the solid curve are for $d=4$ to 6 .

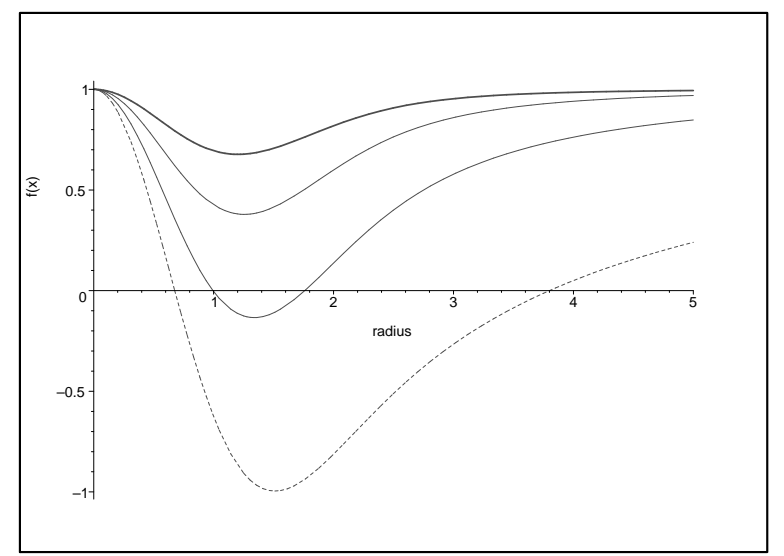

Fig. 21. The noncommutative extradimensional Schwarzschild solution. The function $f(x)$ versus $x=r M_{*}$, for $m=1.9$ and $y=0.5$. With respect the case for $y=0.25$, the noncommutative smearing effect prevails at least for $d \geq 5$, preventing the formation of black holes. In other words, the contraction of the deSitter core is compensated by a quicker expansion of spacetime due to the additional dimensions, which let the mass be more diffused. The dotted curve corresponds to $d=3$, while, contrary to the previous cases, from top to bottom on the left hand side of the figure the solid curve are for $d=6$ to 4 .

intermediate cases, in which the smearing is so strong to prevent the appearance of horizons for higher dimension only, while black holes still occur for $d \lesssim 4$. An anomalous case regards Figs. 23, 24, dealing with a light mass $m \sim 0.475$ and a small $y \sim 0.25$. For $d=3$, we have the extremal black hole, but for higher dimensions, instead of an increase of the smearing effect with consequent absence 


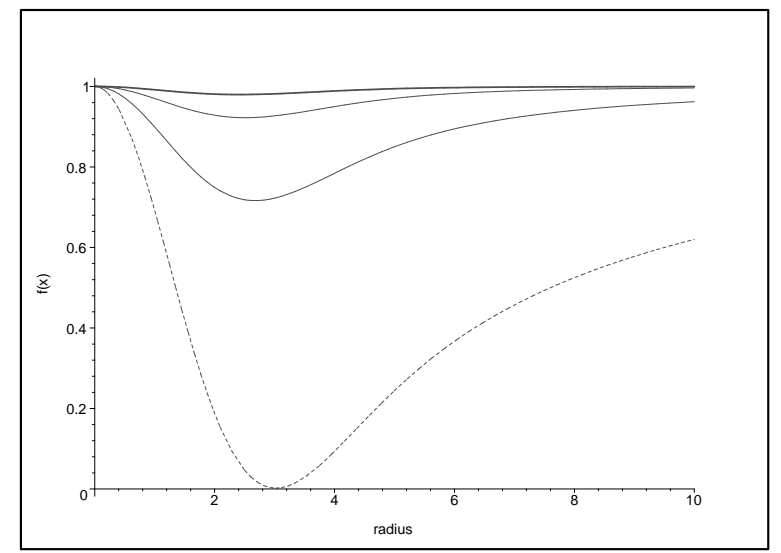

Fig. 22. The noncommutative extradimensional Schwarzschild solution. The function $f(x)$ versus $x=r M_{*}$, for $m=1.9$ and $y=1$. This is a known case at least for $d=3$, for which the extremal back hole takes place. Again the additional dimensions of spacetime determine a further smearing of the mass with absence of black holes for $d \geq 4$ and even of curvature for the higher dimensions. The dotted curve corresponds to $d=3$, while from top to bottom on the left hand side of the figure the solid curve are for $d=6$ to 4 .

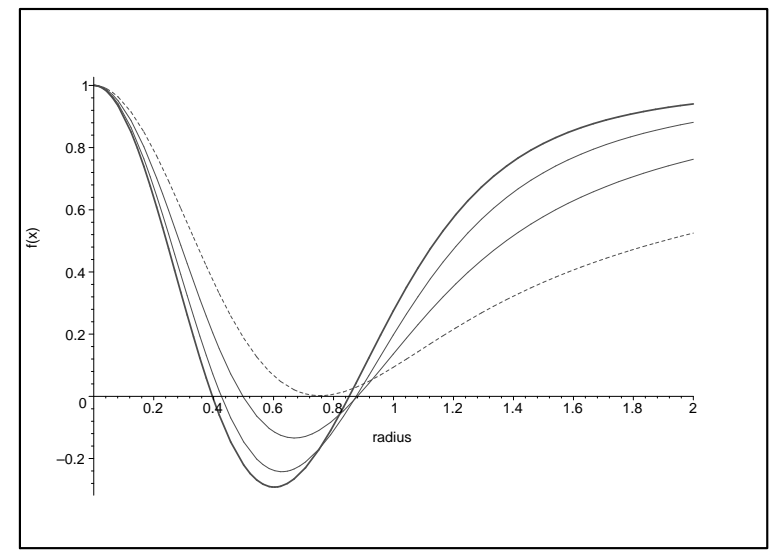

Fig. 23. The noncommutative extradimensional Schwarzschild solution. The function $f(x)$ versus $x=r M_{*}$, for $m=0.475$ and $y=0.25$. For $d=3$ we have the extremal case, while the increase of $d$ prevails on other effects, contracting the deSitter core, which shrinks with $y^{d / 2}$ and reducing and the threshold mass to have a black hole, i.e. $m_{0} \sim y^{d-2}$. A smaller region influenced by noncommutative fluctuations is accompanied by a curvature increase with $y^{-d}$. As a consequence, black holes develop for $d>3$. The dotted curve corresponds to $d=3$, while from top to bottom on the left hand side of the figure the solid curve are for $d=4$ to 6 . We can observe that, this time on the contrary with respect to previous cases, the outer horizon decreases with $d$ from $d=4$.

of horizons, we obtain nonextremal black holes. This fact can be explained by the reduction of the minimal mass in higher dimension, being $m_{\min } \sim x_{\min }^{d-2}(d)$, where $x_{\min }(d)<x_{\min }(3) \approx 0.75$. Geometrically, we can say that a contraction of the 


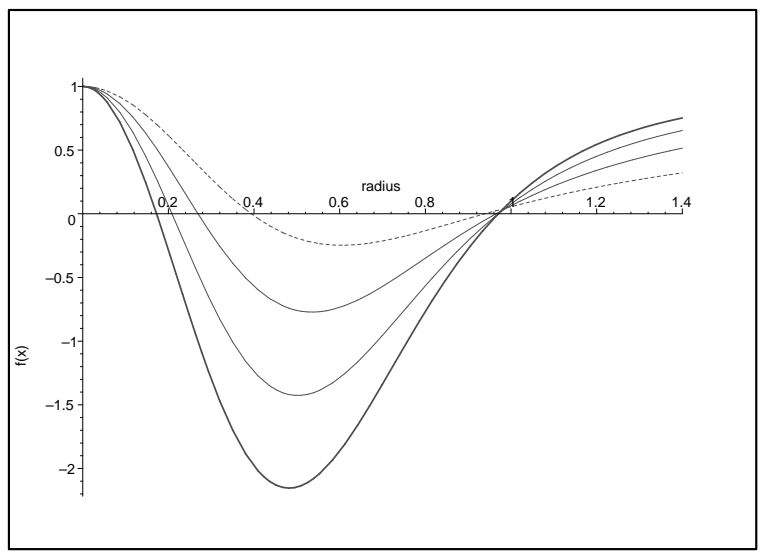

Fig. 24. The noncommutative extradimensional Schwarzschild solution. The function $f(x)$ versus $x=r M_{*}$, for $m=0.475$ and $y=0.2$. With respect to the case $y=0.25$, a reduction of $y$ implies a further contraction of the deSitter core and of the region influenced by noncommutativity, while the curvature increases and the threshold mass decreases. Therefore black holes occur for every $d$, because the spacetime expansion with $d$ cannot compensate the deSitter core reduction: as a consequence, the mass is more diffused as $d$ increases and the the outer horizon increases with $d$. The dotted curve corresponds to $d=3$, while from top to bottom on the left hand side of the figure the solid curve are for $d=4$ to 6 .

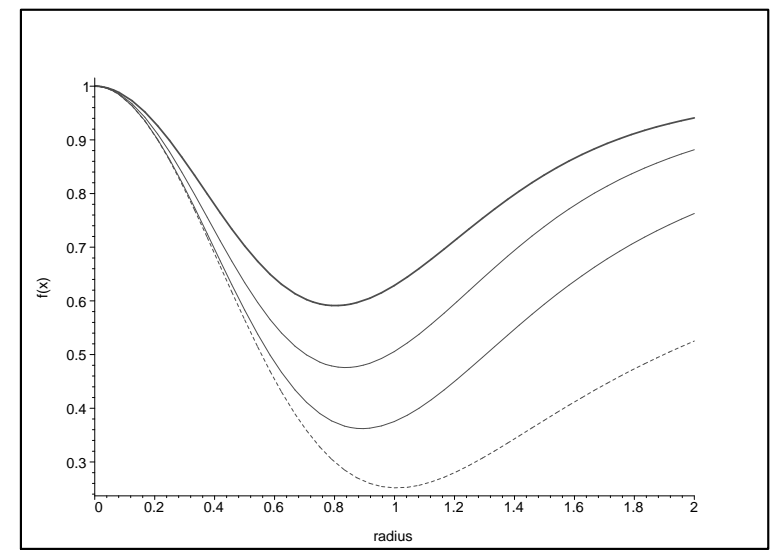

Fig. 25. The noncommutative extradimensional Schwarzschild solution. The function $f(x)$ versus $x=r M_{*}$, for $m=0.475$ and $y=1 / 3$. With respect to the case $y=0.25$, an increase of $y$ implies an expansion of the deSitter core, an increase of threshold masses and a reduction of the curvature. Therefore, even for the $d=3$ case black holes cannot occur. The dotted curve corresponds to $d=3$, while from top to bottom on the left hand side of the figure the solid curve are for $d=6$ to 4 .

deSitter core occurs, i.e. $L \sim y^{d / 2} M_{*}^{-1}$, with a consequent reduction of the region where noncommutativity is relevant and with an increase of curvature near the origin, i.e. $\mathcal{R} \sim y^{-d}$. As a consequence black holes develop for $d>3$ only. A slight 


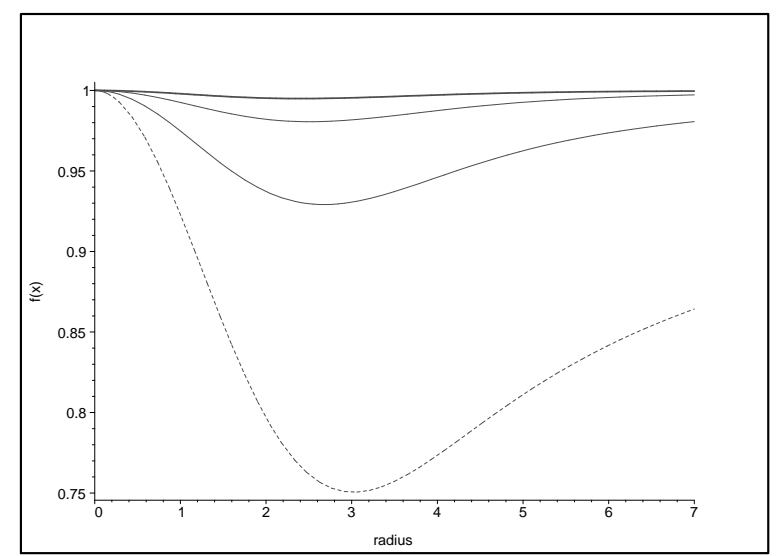

Fig. 26. The noncommutative extradimensional Schwarzschild solution. The function $f(x)$ versus $x=r M_{*}$, for $m=0.475$ and $y=1$. A large value of $y$ determines a manifold dominated by noncommutativity at least in a large region. Other physical parameters turn out to be irrelevant and the $f$ behaves as expected when the diffusion of the mass increase with $d$, but no black hole occurs even for $d=3$. The dotted curve corresponds to $d=3$, while from top to bottom on the left hand side of the figure the solid curve are for $d=6$ to 3 .

reduction of $y$, leads to a fatal reduction of the deSitter core also for $d=3$ with consequent formation of two horizons (see Fig. 24). A special feature of this near extremal case, is that the outer horizon increases with $d$, the contrary of what one generally expects. On the other hand, a small increase of $y$ leads to an opposite scenario in which no horizon occurs for any $d$ (See Fig. 25). Finally in Fig. 26, we find a manifold dominated by noncommutative effects, while the mass $m$ is too light to provide significant gravitational disturbances, in particular for the higher dimensional cases.

\subsubsection{Higher dimensional black hole thermodynamics}

The next step is about the thermodynamical analysis of the noncommutative extradimensional solution. As usual we can calculate the black hole temperature

$$
T_{H}=\frac{d-2}{4 \pi r_{+}}\left[1-\frac{r_{+}}{d-2} \frac{\gamma^{\prime}\left(d / 2, r_{+}^{2} / 4 \theta\right)}{\gamma\left(d / 2, r_{+}^{2} / 4 \theta\right)}\right]
$$

Defining the dimensionless temperature $T \equiv T_{H} / M_{*}$ we have

$$
T=\frac{d-2}{4 \pi x}\left[1-\frac{2}{d-2} \frac{p^{d / 2} e^{-p}}{G_{d}(p) \Gamma(d / 2)}\right]
$$

where again $p=z^{2} / 4$ with $z=x / y$. Then it is instructive to compare this result with the commutative value $T_{\text {comm }}=(d-2) / 4 \pi x$. First of all, both from (116) and from Fig. 27 we can conclude that for large $z$ the black hole temperature coincides with the commutative result. Noncommutative effects appear as $z$ approaches $\sim 6$, 


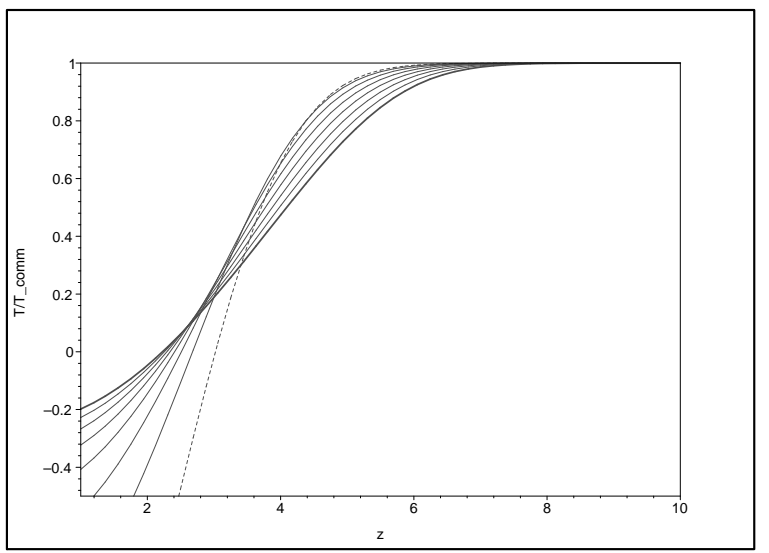

Fig. 27. The noncommutative extradimensional Schwarzschild solution. The ratio $T / T_{\text {comm }}$ as a function of $z$. The dotted curve corresponds to the $d=3$ case whereas, from bottom to top on the left hand side of the figure, the solid lines corresponds to $d=4$ to 10 .

depending on $d$ : the manifold is more sensitive to noncommutativity for higher dimensions, as we can see in the right hand side of Fig. 27, where the lowest curve correspond to the highest dimension. This is in agreement with our previous arguments about the smearing effect, which increases with $d$. The ratio $T / T_{\text {comm }}$ rapidly decreases as $z \sim 3-4$, vanishing at the same values where black hole mass is minimized. Again we see that $z_{\min }$ decreases with $d$. For smaller $z$, we enter negative value of the ordinate corresponding to an unphysical situation.

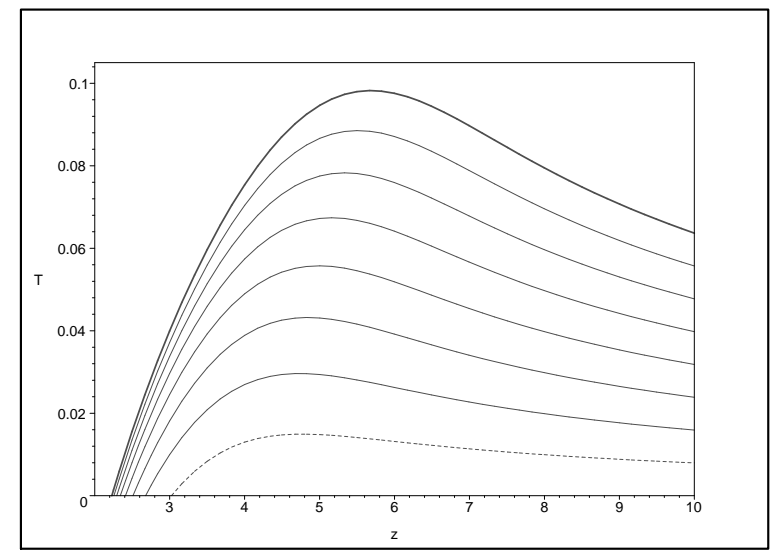

Fig. 28. The noncommutative extradimensional Schwarzschild solution. The temperature $T$ as a function of $z$, for various value of $d$. The dotted curve corresponds to the case $d=3$, while, from the bottom to top on the right hand side of the figure, the solid lines correspond to $d=4$ to 10 . The temperatures increase with $d$, while the SCRAM phase leads to smaller remnant for higher $d$.

Then, from Fig. 28, we conclude that the thermodynamic scenario we have 


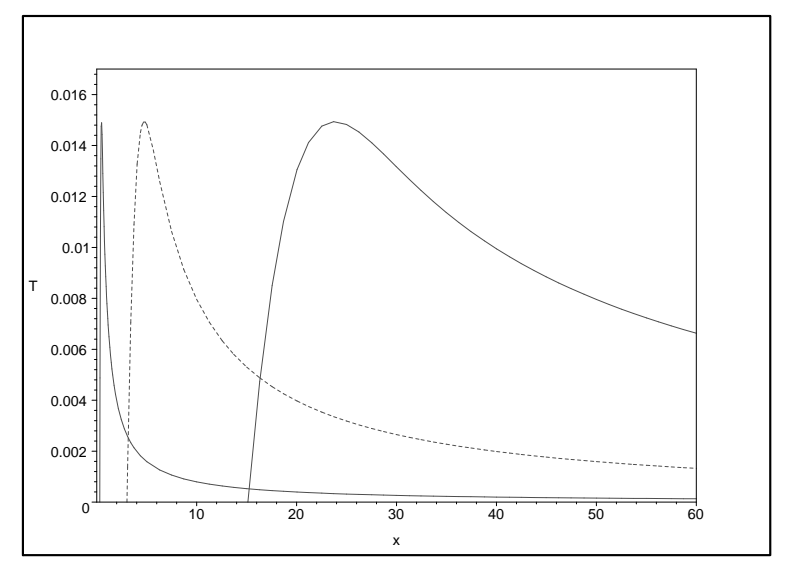

Fig. 29. The noncommutative extradimensional Schwarzschild solution. The temperature $T$ as a function of $x$, for $d=3$. The dotted curve corresponds to the case $y=1$, while, from the bottom to top on the right hand side of the figure, the solid lines correspond to $y=0.1$ and 5 . The temperatures peak is unaffected by $y$. On the other hand smaller values of $y$ reduce the remnant radii, determining a quicker SCRAM phase and a longer Schwarzschild phase.

already encountered with the four dimensional solution, is confirmed in higher dimensions: after an initial Schwarzschild phase, the black hole undergoes a SCRAM phase, ending up with a zero temperature relic. One can see also from Table 2 that the peak of the temperature increases with $d$, but even in the case $d=10$ it is about $98 \mathrm{GeV}\left(\simeq 10^{15} \mathrm{~K}\right)$ which is much lower than $M_{*}$. To this purpose, the parameter $y$ does not play any role in enhancing the temperature peak, determining only a shift of the coordinate $x_{\max }$ where the peak takes place (see Fig. 29). On the other hand, we still have, as reasonable lower bound for the mass parameter, $m_{\min } \sim 1$. As a result, even for the higher dimensional solutions, backreaction effects are negligible: the dimensionless temperature is $T \ll 1 \lesssim m$, as we can see from Table 3 , where for $d=3$ the fundamental scale is $M_{*}=M_{P}$.

Table 2. Black hole maximal temperatures for different $d$. See Fig.28

\begin{tabular}{ccccccccc}
\hline $\mathrm{d}$ & 3 & 4 & 5 & 6 & 7 & 8 & 9 & 10 \\
\hline$T_{H}^{\max }(\mathrm{GeV})$ & $18 \times 10^{16}$ & 30 & 43 & 56 & 67 & 78 & 89 & 98 \\
\hline$T_{H}^{\max }\left(10^{15} \mathrm{~K}\right)$ & $.21 \times 10^{16}$ & .35 & .50 & .65 & .78 & .91 & 1.0 & 1.1 \\
\hline
\end{tabular}

Table 3. Backreaction estimates for different values of $d$

\begin{tabular}{ccccccccc}
\hline $\mathrm{d}$ & 3 & 4 & 5 & 6 & 7 & 8 & 9 & 10 \\
\hline $10^{3} T / m$ & $<15$ & $<30$ & $<43$ & $<56$ & $<67$ & $<78$ & $<89$ & $<98$ \\
\hline
\end{tabular}


In analogy with what we have already seen in the four dimensional case, the stability of remnants, for any $d$, is an obvious consequence of this extradimensional scenario. Indeed the leading term of the mass loss rate may be written as

$$
\frac{d m}{d t} \sim x^{d-1} T^{d+1}
$$

for bulk fields decays. The resulting black hole lifetime is then given by

$$
\text { Life } \sim \int_{m_{\text {initial }}}^{m_{\min }} \frac{d m}{x^{d-1}(m) T^{d+1}(m)}
$$

with $m_{\text {initial }}$ being the starting black hole mass. Recalling that $x(m)$ never vanishes, while $T(m) \rightarrow 0$ as $m \rightarrow m_{\min }$, for all $d$ the lifetime will be driven to infinity, implying a stable relic.

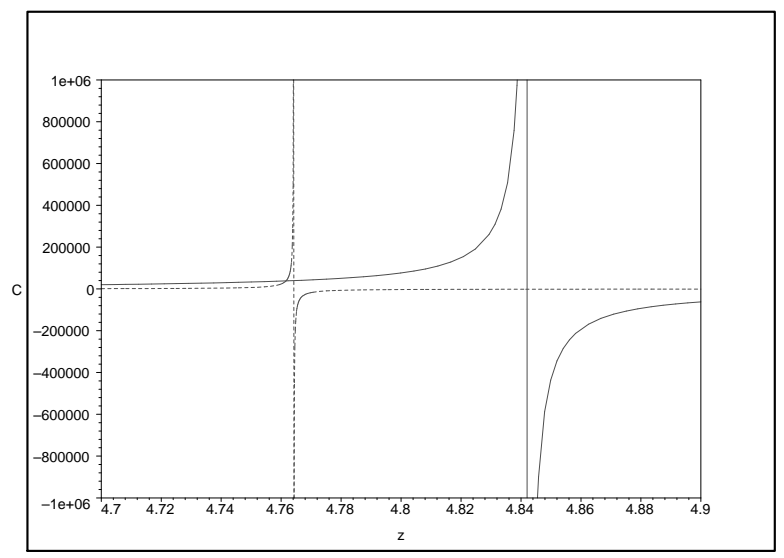

Fig. 30. The noncommutative extradimensional Schwarzschild solution. The heat capacity $C$ as a function of $z$. The dotted curve corresponds to the case $d=3$, while the solid line to $d=5$. At fixed $y$, the noncommutative effects become relevant at larger $z$ for the higher $d$ case; indeed the temperature peaks take place at $\sim 4.76$ and $\sim 4.84$ respectively, determining the heat capacity singularity. On the other hand, from the slope of the curves we get that the SCRAM phase, characterized by positive $C$, is quicker for $d=3$, leading to a larger remnant.

The unexpected temperature behavior in the SCRAM phase suggests the study of the higher dimensional black hole heat capacity

$$
C=\frac{\partial m}{\partial T}=\frac{\partial m}{\partial x}\left(\frac{\partial T}{\partial x}\right)^{-1}
$$

which turns out to be

$$
C=-\frac{2 \pi x^{d-1}}{G_{d}(p)}\left[1+\frac{\frac{4 p H_{d}(p)}{d-2}\left(1+\frac{H_{d}(p)}{p}-\frac{d}{2 p}\right)}{1-\frac{2 H_{d}(p)}{d-2}-\frac{4 p H_{d}(p)}{d-2}\left(1+\frac{H_{d}(p)}{p}-\frac{d}{2 p}\right)}\right]
$$


where for notational purposes we have defined

$$
H_{d}(p)=\frac{p^{d / 2} e^{-p}}{G_{d}(p) \Gamma(d / 2)}
$$

We can see that at large distances, being $G_{d}(p) \rightarrow 0$ and $H_{d}(p) \rightarrow 0$, the heat capacity is negative and approaches the commutative result $\sim-2 \pi x^{d-1}$, while at $x=x_{\min }$ the heat capacity vanishes, because $\partial m / \partial x=0$ in (119). Further we note that the sign of $C$ is uniquely determined by $\partial T / \partial x$, which switches from negative value for $x>x_{\max }$ during the Schwarzschild phase to positive ones for $x_{\min }<x<x_{\max }$ in the SCRAM phase. Such change of sign is accompanied by the appearance of a polar singularity of $C$, which occurs when $\partial T / \partial x$ vanishes at $x=x_{\max }$, i.e. where the temperature reaches the maximum. The Fig. 30 is illuminating about the stability of black hole relics and the behavior of $C$ for different dimensions.

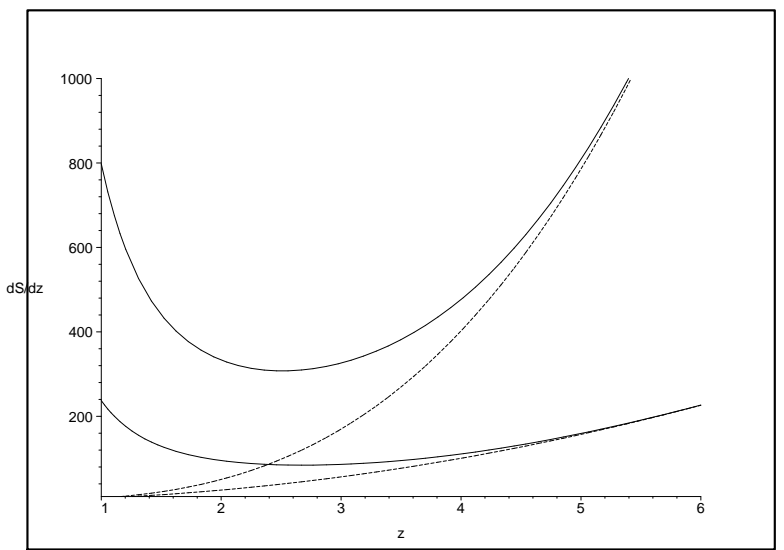

Fig. 31. The noncommutative extradimensional Schwarzschild solution. The derivative of the black hole entropy $d S / d z$ as a function of $z$. The dotted curve correspond to the commutative case, while the upper solid curve regards $d=5$ while the lower $d=4$. In both case, at large distances, $z \sim 5$, the entropy is essentially coincident with its commutative equivalent. In spite of the singularity in the heat capacity and the final zero temperature state, the entropy is always a finite function.

The thermal history of the black hole can be analyzed also in terms of the entropy which can be defined via

$$
S=\int d x T^{-1} \frac{\partial m}{\partial x}=2 \pi \int_{x_{\min }}^{x} t^{d-2} G_{d}^{-1}\left(t^{2} / 4 y^{2}\right) .
$$

In the above definition, we have made the natural choice that $S=0$ at $x_{m i n}$, where the mass $m=m_{\min }$. We note that, in the commutative limit, $y \rightarrow 0$, the function $G_{d} \rightarrow 1$, while the lower limit of integration $x_{\min }=z_{0} y$ vanishes. Thus one recovers 
the conventional result

$$
S_{\text {comm }}=2 \pi \frac{x^{d-1}}{d-1}
$$

corresponding to the large distance behavior of the entropy. The integration of (122) leads to the extra dimensional generalization of (59)

$$
S=2 \pi \frac{x^{d-1}-x_{\min }^{d-1}}{(d-1) G_{d}(z)}+\delta S
$$

where

$$
\delta S \approx \frac{1}{(2 y)^{m}}\left(x_{m i n}^{2 m-3} e^{-x_{m i n}^{2} / 4 y^{2}}-x^{2 m-3} e^{-x^{2} / 4 y^{2}}\right)
$$

is an exponentially suppressed correcting term. If we take into account that the area of the event horizon is $A=2 \pi^{d / 2} x^{d-1} / \Gamma(d / 2)$, we obtain the generalization of the four dimensional entropy-area law to higher dimensions

$$
S=\Gamma(d / 2) \pi^{1-\frac{d}{2}} \frac{\left(A_{+}-A_{e}\right)}{(d-1) G_{d}(z)}+\delta S .
$$

As further note, looking at

$$
S=\int d x \frac{C}{T} \frac{\partial T}{\partial x}
$$

one finds that the entropy is always finite at any critical point of both $C$ and $T$ to get a reliable black hole thermodynamics (see Fig. 31).

Finally, also the free energy can be extended to higher dimensional cases, combining the previous thermodynamic quantities

$$
F=m-T S
$$

which coincides with the commutative value, $F=x^{d-2} / 2(d-1)=m /(d-1)$ at large distances. On the other hand, at $x=x_{\min }$, we have $F=m$ for all value of $y$. At intermediate value of $x$, the free energy decreases, reaching a minimum where the temperature is maximized, namely at $x_{\max }$ where $\partial T / \partial x=0$. After that, the free energy matches onto the asymptotic $\sim x^{d-2}$ behavior. Again the value $x_{\text {max }}$ marks the frontier between an unstable Schwarzschild phase and a stable SCRAM phase, in which a loss of mass implies a decrease of total energy and thermal emission.

As final comment, the present analysis confirms our conjectures: we can expect that black holes be produced with large cross sections and studied in detail at the LHC. In particular, the extradimensional model confirms the four dimensional prevision about the existence of stable remnants, whose mass and radius are fixed by the noncommutative scale and the number of dimensions and whose detection turns out to be vital for the understanding of Quantum Gravity. 


\subsection{The noncommutative extradimensional charged solution}

The final step of this review regards the recently determined noncommutative higher

dimensional charged solution of Einstein's equation 200 . The starting point of any noncommutative quasi classical coordinates is the smearing which fatally affects source terms of equations. To this purpose it has been studied, as preliminary case, the possibility for Standard Model fields to propagate into the bulk rather than being constrained to a four dimensional brane. This strong hypothesis is in agreement with recent arguments in literature within the framework on the Universal Extra Dimensions, that are expected to be compactified at a scale $1 / R$ above few $\mathrm{TeV} 309$. For this reason, one can determine, in the case of spherical symmetry, the quasi classical source terms of both the gravitational and the electromagnetic field, in $D=d+1$ dimensions as

$$
\begin{aligned}
\rho_{\text {matt }}(r) & =\frac{\mathcal{M}}{(4 \pi \theta)^{d / 2}} \exp \left(-r^{2} / 4 \theta\right) \\
\rho_{\text {el }}(r) & =\frac{\mathcal{Q}}{(4 \pi \theta)^{d / 2}} \exp \left(-r^{2} / 4 \theta\right) .
\end{aligned}
$$

Such sources are going to describe the noncommutative behavior of the electrogravitational system within the framework of the quasi classical set of field equations

$$
\begin{aligned}
& R_{N}^{M}-\frac{1}{2} \delta_{N}^{M} \mathcal{R}=\frac{8 \pi}{M_{*}^{d-1}}\left(\left.T_{N}^{M}\right|_{\text {matt. }}+\left.T_{N}^{M}\right|_{\text {el. }}\right) \\
& \frac{1}{\sqrt{-g}} \partial_{M}\left(\sqrt{-g} F^{M N}\right)=J^{M}
\end{aligned}
$$

with $M, N=0, \ldots, d$. The tensors $\left.T_{N}^{M}\right|_{\text {matt. }}$ and $\left.T_{N}^{M}\right|_{e l}$. are the higher dimensional equivalent of the energy momentum tensors, describing the matter and the electromagnetic content, in the four dimensional solution 238 .

The above Maxwell field can be obtained as

$$
F^{M N}=\delta^{0[M \mid} \delta^{r \mid N]} E(r)
$$

where $E(r)$ is the electric field produced by the charge distribution $\rho_{e l}(r)$

$$
E(r)=\frac{1}{\pi^{(d-2) / 2}} \frac{2 \mathcal{Q}}{r^{d-1}} \gamma\left(\frac{d}{2}, \frac{r^{2}}{4 \theta}\right),
$$

while the corresponding current density is

$$
J^{M}(x)=4 \pi \rho_{e l}(r) \delta_{0}^{M} .
$$

We note that the electric field results regular and vanishing at the origin for any $d$, while at large distances it exhibits the conventional $1 / r^{d-1}$ behavior. This is a further check of consistency of the quasi coordinates approach to noncommutative geometry. For the above conditions, we assume a metric of the following form

$$
d s_{(d+1)}^{2}=-f(r) d t^{2}+f^{-1}(r) d r^{2}+r^{2} d \Omega_{d-1}^{2}
$$




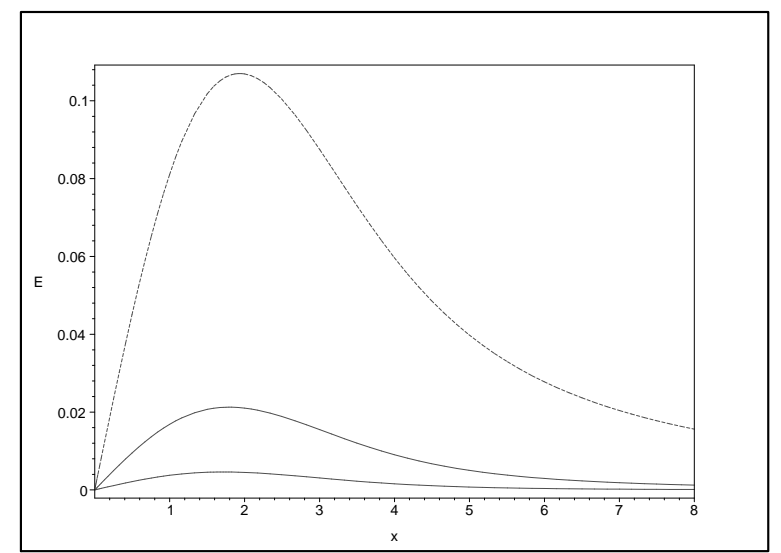

Fig. 32. The noncommutative extradimensional charged solution. The electric field $E(x)$ as a function of $x=r M_{*}$ for $e=1 \times M_{*}^{(d-3) / 2}$ and $y=M_{*} \sqrt{\theta}=1$. The electric field reaches its maximum intensity near $x \approx 2$, and then drops to zero for any $d$. An increase of $d$ determines a weaker field, since the spacetime turns out to be larger. Regarding the long distance behavior, we find that of ordinary Coulomb field, $1 / x^{d-1}$.

with the usual demand that $f(r) \rightarrow 1$ as $r \rightarrow \infty$, while $d \Omega_{d-1}$ is still the surface element of $d$-1-dimensional unit sphere. Plugging the above line element into the Einstein-Maxwell system, we can determine the function $f(r)$

$$
f(r)=1-\frac{1}{M_{*}^{d-1}} \frac{4 \mathcal{M}}{\pi^{(d-2) / 2} r^{d-2}} \gamma\left(\frac{d}{2}, \frac{r^{2}}{4 \theta}\right)+\frac{d-2}{M_{*}^{d-1}} \frac{4 \mathcal{Q}^{2} F_{d}(r)}{\pi^{d-3} r^{2 d-4}}
$$

where

$$
F_{d}(r) \equiv \gamma^{2}\left(\frac{d}{2}-1, \frac{r^{2}}{4 \theta}\right)-\frac{2^{(8-3 d) / 2} r^{d-2}}{(d-2) \theta^{(d-2) / 2}} \gamma\left(\frac{d}{2}-1, \frac{r^{2}}{2 \theta}\right)
$$

In analogy of what we have already seen in the four dimensional case, the resulting manifold is curvature singularity free. Indeed the short distance geometry is governed by a deSitter line element, whose effective cosmological constant coincides with what found in the neutral extradimensional case

$$
\Lambda=\frac{1}{M^{d-1}} \frac{4 \mathcal{M}}{d 2^{d-1} \pi^{(d-2) / 2} \theta^{d / 2}} .
$$

This feature enlightens the validity of the present method and represents a matter to reject all the other noncommutative geometry approaches. Introducing the total mass energy, due to both matter and electromagnetic field energy contributions

$$
M(r)=\frac{2 \mathcal{M}}{\pi^{\frac{d-2}{2}}} \gamma\left(\frac{d}{2}, \frac{r^{2}}{4 \theta}\right)+\frac{2^{\frac{8-3 d}{2}}}{\pi^{d-3}} \frac{2 \mathcal{Q}^{2}}{\theta^{\frac{d}{2}-1}} \gamma\left(\frac{d}{2}-1, \frac{r^{2}}{2 \theta}\right)
$$

and its asymptotic limit $M$, namely the total mass energy as measured by an asymptotic observer

$$
M=\lim _{r \rightarrow \infty} M(r)=\frac{2 \mathcal{M}}{\pi^{\frac{d-2}{2}}} \Gamma\left(\frac{d}{2}\right)+\frac{2^{\frac{8-3 d}{2}} 2 \mathcal{Q}^{2}}{\pi^{d-3} \theta^{\frac{d-2}{2}}} \Gamma\left(\frac{d}{2}-1\right),
$$


one can write the above line element as

$$
\begin{aligned}
f(r) & =1-\frac{1}{M_{*}^{d-1}} \frac{2 M}{r^{d-2} \Gamma\left(\frac{d}{2}\right)} \gamma\left(\frac{d}{2}, \frac{r^{2}}{4 \theta}\right) \\
& +\frac{4 \mathcal{Q}^{2}(d-2)}{M_{*}^{d-1} \pi^{d-3} r^{2 d-4}}\left[F_{d}(r)+c_{d}\left(\frac{r}{\sqrt{\theta}}\right)^{d-2} \gamma\left(\frac{d}{2}, \frac{r^{2}}{4 \theta}\right)\right]
\end{aligned}
$$

where the coefficient $c_{d}$ is given by

$$
c_{d} \equiv \frac{2^{\frac{8-3 d}{2}}}{d-2} \frac{\Gamma\left(\frac{d}{2}-1\right)}{\Gamma\left(\frac{d}{2}\right)} .
$$

This line element nicely behaves at large distances too: indeed for $r \gg \sqrt{\theta}$ we have

$$
f(r) \sim 1-\frac{1}{M_{*}^{d-1}} \frac{2 M}{r^{d-2}}+\frac{4 \mathcal{Q}^{2}(d-2)}{M_{*}^{d-1} \pi^{d-3} r^{2 d-4}} \Gamma^{2}\left(\frac{d}{2}-1\right),
$$

namely the line element (142) asymptotically reproduces the ordinary ReissnerNordström geometry.

\subsubsection{The horizon equation}

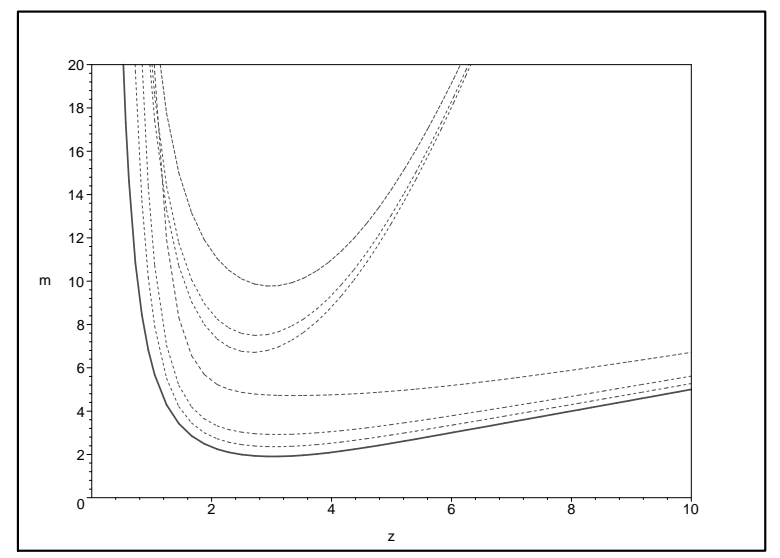

Fig. 33. The noncommutative extradimensional charged solution. The mass function $m$ versus the horizon radius $z$ for different $q$ 's and $d$ 's and $y=1$. The solid curve represent the neutral three dimensional case, while the the dashed curves, from the bottom to the top, are respectively for the cases $d=3, q=2 ; d=3, q=3 ; d=3, q=5 ; d=4, q=1 ; d=4, q=10 ; d=4, q=20$. At first glance we can conclude that minimal masses increase with the charge $q$ and the number of dimensions $d$, while their radii decreases with $d$ and increase with $q$.

At this point, we can study the existence of the horizon solving the equation $f(r)=0$. For sake of notational clearness, it is worthwhile to introduce dimensionless quantities, such as $m=M / M_{*}, x=r_{H} M_{*}, y=M_{*} \sqrt{\theta}$ and $q=\mathcal{Q} M_{*}^{(d-3) / 2}$ and 
write the horizon equation as

$$
2 m G_{d}(z)=x^{d-2}+a_{d} q^{2} x^{2-d}\left[F_{d}(z)+b_{d} z^{d-2} G_{d}(p)\right]
$$

where we recall the definition of effective Newton constant

$$
G_{d}(p)=\frac{1}{\Gamma(d / 2)} \gamma(d / 2, p)
$$

with $z=x / y, p=z^{2} / 4$, while

$$
a_{d}=\frac{4(d-2)}{\pi^{d-3}}
$$

and $b_{d}=\Gamma(d / 2) c_{d}$. The function $F_{d}$ can be written in a more convenient form as

$$
F_{d}(z)=\Gamma^{2}(d / 2-1) G_{d-2}^{2}(p)-b_{d} z^{d-2} G_{d-2}(p)
$$

where $p=z^{2} / 2$.

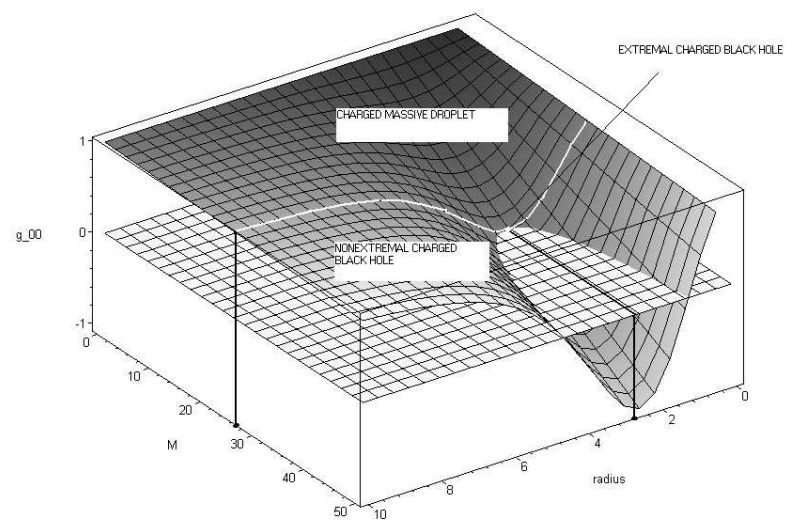

Fig. 34. The noncommutative extradimensional charged solution. The function $f$ versus $m$ and $z$, for a charge, $q=1$ and $d=4$. With respect to the $d=3$ case, the intersection with the $f=0$ plane (light grey) occurs at larger value of both $m$ and $z$.

We can see that the total mass energy diverges both at short distances $m \sim z^{-2}$ and at large distances $m \sim z^{d-2}$. Therefore we do expect the existence of a minimum value of $m$ at some intermediate value of $x$, in agreement with Figs. 33 and 34. It is also evident that for any $d$ there can be two horizons when $m>m_{\min }$, one degenerate horizon for $m=m_{\min }$, or no horizon in $m<m_{\min }$. Again, from the study of the temperature, the mass $m_{\min }$ of the extremal black hole, is expected to be the end of the Hawking evaporation process. The analysis of the neutral case showed that black holes are too massive to be produced at LHC for $y$ about 1. In the charged case the situation is even worse. In Fig. 33, families of curves labelled by the value of $d$ and assigned value of the electric charge $q$. The horizons, 
and the corresponding values of $m$, can be read as the intersections of the grid lines, with each curve in Fig.(34). The minimum of the curves defines the extremal black hole configurations in different dimensions. We see that the effect of both extradimensions and charges is to lift upward the curves, increasing the value of the mass for a given radius of the horizon, including the degenerate case. Therefore, we conclude that the evolution of the black hole towards its extremal configuration is qualitatively the same as described in four dimensional case 162 . The increase of the mass $m_{\min }$ and its eventual experimental verification could indicate the number of extra-dimensions. On the other hand, our current experimental ability would demand lower value for the masses and therefore $y \sim 0.1$. Further on smaller $y$ would lead to the commutative limit, in which the horizon equation becomes

$$
2 m=x^{d-2}+a_{d} q^{2} x^{2-d} \Gamma^{2}(d / 2-1)
$$

whose roots are given by

$$
x_{ \pm}^{d-2}=m \pm\left(m^{2}-a_{d} q^{2} \Gamma^{2}(d / 2-1)\right)^{1 / 2} .
$$

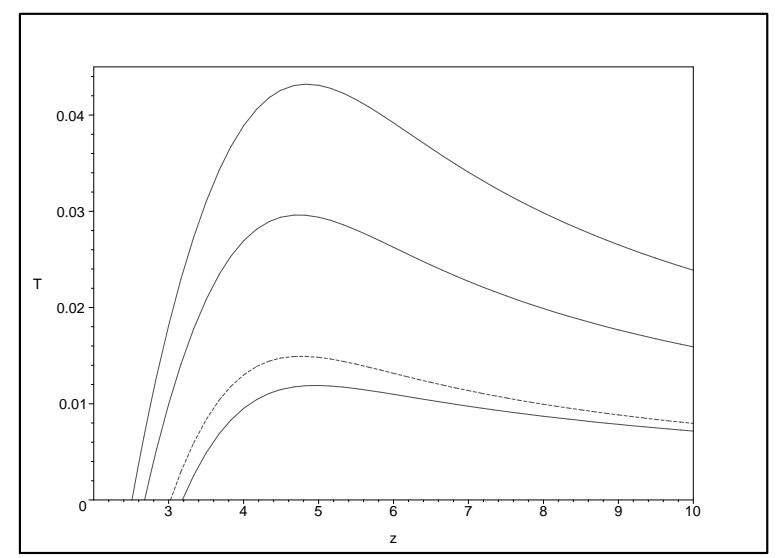

Fig. 35. The noncommutative extradimensional charged solution. The dimensionless Hawking temperature $T$ as a function of $z$, for different values of $q$ and $d$. The dashed curve regards the case $d=3, q=0$, while the solid curves from the bottom to the top regard the cases $d=3 q=1$, $d=4 q=1$ and $d=5 q=1$. In all such cases $T$ reaches a maximum whose value increases with $d$ before dropping to zero. The role of the charge is just to lower down the temperature at any $z$.

\subsubsection{Higher dimensional charged black hole thermodynamics}

On the thermodynamics side, the temperature is going to reveal the behavior of the black hole in the so called balding phase. The four dimensional analysis showed that very quickly the black hole reaches the Schwarzschild phase and thus subsequent SCRAM basically occurs in a neutral regime. In other words, Schwinger pair production and Hawking emission behave like two temporally distinct processes. At 
this point, it is imperative to know whether this four dimensional scenario could be altered by the presence of extradimensions. The starting point is the explicit form of the Hawking temperature

$$
\begin{aligned}
T_{H} & =\frac{d-2}{4 \pi r_{+}}\left[1-\frac{r_{+}}{d-2} \frac{\gamma^{\prime}\left(d / 2, r_{+}^{2} / 4 \theta\right)}{\gamma\left(d / 2, r_{+}^{2} / 4 \theta\right)}\right]+ \\
& -\frac{16 \mathcal{Q}^{2}(d-2)}{M_{*}^{d-1} \pi^{d-3} r_{+}^{2 d-3}}\left[\frac{1}{d-2} \gamma^{2}\left(d / 2, r_{+}^{2} / 4 \theta\right)+F\left(r_{+}\right) \frac{r_{+}}{4} \frac{\gamma^{\prime}\left(d / 2, r_{+}^{2} / 4 \theta\right)}{\gamma\left(d / 2, r_{+}^{2} / 4 \theta\right)}\right]
\end{aligned}
$$

where, we replaced $M$ with $r_{+}$by using the horizon equation. The dimensionless form of the above formula reads

$$
T=\frac{d-2}{4 \pi x}\left[1-\frac{2}{d-2} H_{d}(p)\right]-\frac{16 q^{2}(d-2)}{\pi^{d-3} x^{2 d-3}}\left[\frac{\Gamma^{2}(d / 2) G_{d}^{2}(p)}{d-2}+\frac{1}{2} F_{d}(p) H_{d}(p)\right]
$$

where $H_{d}=p^{1 / 2} e^{-p} / G_{d}(p) \Gamma(d / 2)$. It is clear that the additional term due to the charge may become important for small $x$ only. Indeed from Fig. 35, one learns that the temperature behavior is equivalent to that encountered in the neutral case. The effect of the charge is just to lower down the already regular curve of the temperature. Also the entropy resembles that encountered in the previous cases. Both from

$$
d m=T d S+\frac{\partial m}{\partial q} d q
$$

and

$$
d m=\frac{\partial m}{\partial z} d z+\frac{\partial m}{\partial q} d q
$$

one gets

$$
d S=\frac{1}{T}\left(\frac{\partial m}{\partial z}\right) d z
$$

Therefore, calculating $\partial m / \partial z$ and integrating from $x_{\min }$ to a generic $x$, one again finds

$$
S=\Gamma(d / 2) \pi^{1-\frac{d}{2}} \frac{\left(A_{+}-A_{e}\right)}{(d-1) G_{d}(z)}+\delta S,
$$

in analogy to what already seen for the four dimensional charged case and the extradimensional neutral case. In the present case, the event horizon areas $A=$ $2 \pi^{d / 2} z^{d-1} / \Gamma(d / 2)$ do depend on both $d$ and $q$ through $z$. Again, one can find that the correcting term $\delta S$ is negligible and the area entropy law still holds up to exponentially suppressed corrections. 


\subsubsection{Schwinger mechanism and dyadosphere}

At this point, we still have to study the discharge time of the black hole. First of all, the analysis in the four dimensional case about the development of the dyadosphere can be extended to the extradimensional case and leads to the same conclusions. The condition to have a pair producing field implies

$$
\frac{2 \mathcal{Q}}{\pi^{\frac{d-2}{2}} r^{d-1}} \gamma\left(\frac{d}{2}, \frac{r^{2}}{4 \theta}\right) \geq \frac{m_{e}^{2}}{e}
$$

where $m_{e}$ and $e$ are the mass and the charge of each particle of the pair. Supposing that the black hole total charge is $\mathcal{Q}=Z e$, a multiple of the elementary charge $e$, we have $q=\mathcal{Q} M_{*}^{(d-3) / 2}=Z$ and we can conveniently write the above relation in term of dimensionless quantities as

$$
\frac{1}{y^{d-1}} \frac{2 Z}{\pi^{(d-2) / 2} z^{d-1}} \gamma\left(\frac{d}{2}, \frac{z^{2}}{4}\right) \geq\left(\frac{m_{e}}{M_{*}}\right) \sim 2.5 \times 10^{-13}
$$

Therefore, for any $y \leq 10$ and for any $d=3-10$, we can conclude that it is sufficient a single elementary charge, $Z=1$, to have Schwinger pair creation at the black hole event horizon. In other words it is very likely that dyadospheres, the spherical surface where the above condition is satisfied, have radii even larger than $r_{+}$. To this purpose it is crucial to know whether such dyadosphere can be formed. According to 255 257, dyadospheres develop only if

$$
\frac{E}{E_{t h}}=\frac{e}{r^{d-1} m_{e}^{2}} \frac{\mathcal{Q}}{\Gamma(d / 2)} \gamma\left(\frac{d}{2}, \frac{r^{2}}{4 \theta}\right) \ll \frac{m_{p} M G}{r^{d-1} m_{e}^{2}} \sim\left(\frac{m_{p}}{m_{e}}\right) \frac{M}{m_{e}} \sim 4 \times 10^{9}
$$

where $E_{t h} \equiv m_{e}^{2} / e$ is the Schwinger threshold field. Here we see that the above condition which is always met in any dimension, being $m_{p}$ the proton mass. This means that the electric field may be strong enough to produce pairs without the electrostatic repulsion overcoming the gravitational attraction among collapsing hadronic partons. At first order, we find that the dyadosphere radius $r_{d s}$ is

$$
r_{d s}^{d-1} \simeq \frac{2 e \mathcal{Q}}{\pi^{(d-2) / 2} m_{e}^{2}} \gamma\left(\frac{d}{2}, \frac{r_{0}^{2}}{4 \theta}\right)
$$

where $r_{0}$ is the $0^{t h}$ order result, given by

$$
r_{0}^{d-1}=\frac{2 e \mathcal{Q}}{\pi^{(d-2) / 2} m_{e}^{2}} \Gamma\left(\frac{d}{2}\right) .
$$

At this point, we can calculate the black hole discharge time. To this purpose, let us introduce the surface charge density

$$
\sigma(r)=\frac{1}{A} \frac{\mathcal{Q}}{\Gamma(d / 2)} \gamma\left(\frac{d}{2}, \frac{r^{2}}{4 \theta}\right)=\frac{\mathcal{Q}}{2 \pi^{d / 2} r^{d-1}} \gamma\left(\frac{d}{2}, \frac{r^{2}}{4 \theta}\right)
$$

and divide the dyadosphere in thin shells, whose width is the electron Compton wave length $\lambda_{e}=1 / m_{e}$. Thus, the resulting electric field $E(r)=4 \pi \sigma(r)$ can be considered 
constant within each of such shells and we are allowed to apply Schwinger formula

$$
W=\frac{(e E)^{(d+1) / 2}}{2^{d+1} \pi^{d}} \exp \left(-\frac{\pi m_{e}^{2}}{e E}\right)
$$

where $W$ is the pair production rate per volume. As a consequence, the total number of pairs produced in the time interval $\Delta \tau$ turns out to be

$$
\begin{aligned}
\Delta N & \equiv \lambda_{e} A(r) W \Delta \tau \\
& =\frac{\lambda_{e} r^{d-1} m_{e}^{d+1}}{2^{d} \pi^{d / 2} \Gamma\left(\frac{d}{2}\right)}\left(\frac{\sigma}{\sigma_{c}}\right)^{(d+1) / 2} \exp \left(-\pi \frac{\sigma_{c}}{\sigma}\right) \Delta \tau
\end{aligned}
$$

where the threshold density $\sigma_{t h}=m_{e}^{2} / 4 \pi e$ is obtained when $E=E_{t h}$. The integration of the above formula has to be done assuming that the discharge process takes place till $\sigma$ reaches the threshold value $\sigma_{t h}$. As a result we have

$$
\Delta \tau=\frac{\theta m_{e}}{\alpha_{e m}}\left(\frac{2 \pi}{m_{e} c \sqrt{\theta}}\right)^{d-1} \frac{s-1}{s^{(d+1) / 2}} \exp \left(\frac{\pi}{s}\right)
$$

where $s=\sigma / \sigma_{t h}$ and $\alpha_{e m}=1 / 137$ is the fine structure constant in four dimensions. The dimensionless version of the above relation reads

$$
\Delta t=\frac{1}{y^{d-3}} \frac{m_{e} / M_{*}}{\alpha_{e m}}\left(\frac{2 \pi}{m_{e} / M_{*}}\right)^{d-1} \frac{s-1}{s^{(d+1) / 2}} \exp \left(\frac{\pi}{s}\right)
$$

where $\Delta t=\Delta \tau M_{*}, m_{e} / M_{*} \approx 5 \times 10^{-7}$ and, as usual, $y=\sqrt{\theta} M_{*}$. The Eqs. (165) and (166) give the discharge time assuming that the process occurs in the $d+$ 1 dimensional bulk spacetime. To this purpose, it is interesting to compare the extradimensional time $\Delta \tau$ to $\Delta \tau_{d=3}$, the corresponding one for $d=3$. In particular, being $\Delta \tau_{d=3} \leq 1.76 \times 10^{-19} \mathrm{~s}$, we can conclude that

$$
\Delta \tau \leq \frac{1}{y^{d-3}}\left(\frac{2 \pi}{m_{e} / M_{*}}\right)^{d-3} 1.76 \times 10^{-19} \mathrm{~s} .
$$

As a consequence, if the pair production takes place in the bulk, the discharge time significantly increases with $d$, while, according to the brane universe scenario in which only gravity can probe transverse higher dimensions, $\Delta \tau=\Delta \tau_{d=3} \leq$ $1.76 \times 10^{-19} \mathrm{~s}$. In the latter case, the noncommutative charged solution can be efficiently employed in brane models: indeed quickly the black hole turns into the noncommutative Schwarzschild one, whose event horizon radius is small with respect to the size of the extradimensions and let us adopt the spherical symmetry condition to describe the subsequent thermal emission, even in the presence of a $D$-brane.

Table 4. Schwinger discharge time upper bounds, for different $d$ and $y=1$

\begin{tabular}{ccccccccc}
\hline $\mathrm{d}$ & 3 & 4 & 5 & 6 & 7 & 8 & 9 & 10 \\
\hline$\Delta \tau$ (seconds) & $10^{-19}$ & $10^{-12}$ & $10^{-5}$ & $10^{2}$ & $10^{9}$ & $10^{16}$ & $10^{23}$ & $10^{30}$ \\
\hline
\end{tabular}


Table 5. Schwinger discharge time upper bounds, for different $d$ and $y=10$

\begin{tabular}{ccccccccc}
\hline $\mathrm{d}$ & 3 & 4 & 5 & 6 & 7 & 8 & 9 & 10 \\
\hline$\Delta \tau$ (seconds) & $10^{-19}$ & $10^{-13}$ & $10^{-7}$ & $10^{-1}$ & $10^{5}$ & $10^{11}$ & $10^{17}$ & $10^{23}$ \\
\hline
\end{tabular}

From Eq. 167 and Tables 4, 5, it emerges that Schwinger mechanism is relevant for larger $y$, while becomes negligible in the commutative regime, namely for $y \ll 1$. In particular, the Schwinger mechanism dominates the Hawking emission in the early stages of the black hole life for $d \leq 5, d=1$ and $d \leq 6, y=10$.

\subsubsection{Phenomenological implications}

As final comment, we showed that the noncommutative extradimensional charged solution basically reproduces all the main feature of the previous noncommutative solution. Anyway, the presence of the charge is important because since we do expect the production of charged black holes that might be surrounded by clouds of opposite charges. At hadron colliders, such as LHC, generally electrons are more easily seen among other hadronic particles. Therefore, the presence of many electrons near an object among the hadronic fragments, could provide a further signal in support to the creation of a mini (charged) black hole, even if, in case of quick discharge time, its remnant is expected to be neutral.

\section{Future Perspectives}

This review represents both a summary of the state of the art and a starting point towards the exciting arena of new physical phenomena that may be observed in the next few months at the CERN Large Hadron Collider and in subsequent experiments. We have merged together some basic ingredients including Hawking radiation phenomenology, General Relativity, Particle Physics, Noncommutative Geometry and $\mathrm{TeV}$ Quantum Gravity in order to make a reliable picture of the nature of mini black holes when noncommutative effects are taken into account. Indeed, black hole production at the LHC may be one of the early signatures of the validity of the models which we have been reviewing and, more in general, of Noncommutative Geometry and TeV Quantum Gravity.

In particular, we have provided a detailed analysis of all of the existing black hole models within a noncommutative background spacetime. For doing this, we introduced the most popular formulations of Noncommutative Geometry, with particular emphasis on those which lead to mathematically tractable field equations for gravity and hence to physically meaningful solutions. We presented all of the different kinds of line element ever proposed in the noncommutative literature and proceeded by following those approaches which, by an efficient introduction of a natural cutoff in spacetime, have successfully reached the goal of removing the singular short distance behavior of black hole solutions in General Relativity. This has 
really been an unavoidable requirement for two reasons: first, at phenomenological level, we need a tool that lets us describe the final stages of black hole evaporation and predict finite, experimentally testable values for temperatures, masses, cross sections and lifetimes; second, there is no reason to justify the effort in formulating and adopting a noncommutative background spacetime if the Planck phase of the evaporation is plagued by the same divergences and singularities as in the conventional method based on the usual differential spacetime manifold. With this in mind, we showed that the approach based on quasi-classical noncommutative coordinates is able to implement the nonlocal character of noncommutativity for any field theory propagating on the noncommutative manifold, including gravity. This approach let us spread out any point-like source in the field equations, determining the exact form of the noncommutative smearing in terms of Gaussian distributions, whose width is governed by the minimum length $\sqrt{\theta}$, induced by

$$
\left[\mathbf{x}^{i}, \mathbf{x}^{j}\right]=i \theta^{i j},
$$

the fundamental relation of Noncommutative Geometry. Furthermore, we have seen that the quasi-coordinates approach leads to field equations which formally resemble the conventional classical equations: for this reason, our analyses of black hole solutions in four dimensions, for both the neutral and charged cases, could appear, at first sight, as new nonsingular line elements of ordinary General Relativity. By itself, this could already be considered as being a nontrivial result, but there is much more. After a deeper inspection, new features arise that can only be explained in terms of quantum geometrical effects. Indeed, the fate of the evaporating black hole is drastically modified by Noncommutative Geometry and in place of the conventional Planck phase, a new final phase emerges, the SCRAM phase, during which the black hole, after a temperature peak, cools down to a stable zero-temperature remnant configuration. A conclusion of this kind for the black hole's life would not only provide a concrete answer to the long-standing problem of information loss, but would also be the signature for the experimental evidence of mini-black hole production. In particular, once we abandon the conventional four-dimensional spacetime, ruled by the unreachable Planck scale $M_{P}$, the possibility of producing mini-black holes and therefore of finding their remnants at $M_{*} \sim 1 \mathrm{TeV}$, becomes more than a wild conjecture. Experimentally, this implies that we should detect a suppression of di-jet events above the black hole production threshold energy, because the Hawking radiation is expected to mask the standard scattering in the energy range of the emission 314 . Of course this consideration has to be reviewed in the light of the noncommutative solutions, which imply lower upper bounds for the peak of the temperature and therefore a modified scenario for the decay, taking for granted that black holes can be produced in hadronic collisions. Indeed, all that we know about the particles emitted during the evaporation is based on the conventional Schwarzschild solution 315 . An analysis of this kind can still be applied before the SCRAM phase, namely until the Hawking temperature reaches $T_{H} \lesssim T_{H}^{\max }$, where the temperature peak $T_{H}^{\max }$ is around $30-100 \mathrm{GeV}$ depending on the number of 
extra dimensions. In particular, it is believed that a $D$-dimensional black hole can emit energy and angular momentum both in the $(3+1)$ brane universe and in the $D$-dimensional bulk, where the brane is embedded. Some estimates suggest that the black hole radiates mainly on the brane, even if almost half of the total energy is lost in the bulk 316 where direct observations are not possible. The brane emission is governed by

$$
\langle N\rangle_{\omega s}=\frac{|A|^{2}}{e^{\omega / T_{H}}-(-1)^{2 s}}
$$

where $\langle N\rangle_{\omega s}$ is the average number of particles with energy $\omega$ and spin $s$, emitted by a Schwarzschild black hole with temperature $T_{H}$, while $|A|^{2}$ is the greybody factor taking into account the gravitational potential barrier in the vicinity of the horizon. Integration of the above formula lets us determine the contribution of spin $s$ Standard Model particles to the power and flux emitted on the brane. It has been shown that $\sim 75 \%$ of the decay products are quarks, anti-quarks and gluons, while only $\sim 12 \%$ are charged leptons and photons $\frac{41}{}$. Because of this, since the dominant parton contribution cannot be directly observed, one must take into account all of the QED/QCD interactions, including hadronization, in order to understand the kind of spectra that we are going to detect. In particular, a lot of attention has been devoted to the presence of quark-gluon plasma around the black hole before hadronization takes place 320 . Indeed the parton density near to the event horizon grows like $T_{H}^{3}$ : if the temperature is high enough, namely higher than a critical value $T_{c}$, one expects interactions among quarks and gluons through bremsstrahlung and pair production processes. These $2 \rightarrow 3$ processes can increase the number of quarks and gluons, determining the onset of a quark/gluon plasma appearing in the vicinity of the horizon. Therefore propagation through this plasma is affected by a loss of energy which leads to the recombination of partons into hadrons. Also photons, electrons and positrons are equally subject to these processes when QED is considered. For this reason it is worthwhile to define two regions surrounding the black hole, the photosphere where QED mechanisms lead to a $e^{ \pm}, \gamma$ plasma, and the chromosphere, where QCD mechanisms lead to a quark-gluon plasma. Both regions have been studied numerically by solving the Boltzmann equation for the distribution function of the interacting particles, assuming that scattering processes become important only beyond some critical temperature, $T_{c}^{Q E D} \sim 50 \mathrm{GeV}$ for the photosphere and $T_{c}^{Q C D} \sim 175 \mathrm{MeV}$ for the chromosphere. On the other hand, these considerations hold only in the vicinity of the horizon: when interactions of the emitted particles are properly considered, the emission spectrum at the photo/chromosphere edge is modified and the black hole behaves like a black body at a lower temperature. It is clear that the above analysis of photosphere development has to be revised when the black hole has a maximal temperature, as in the noncommutative case. However the considerations about the chromosphere seem to still be valid in the noncommutative scenario and it is reasonable to have $T_{H} \gtrsim \Lambda_{Q C D} \gtrsim T_{c}^{Q C D}$ leading to a strongly coupled quark-gluon plasma and final spectra dominated by hadrons. 
In connection with this, we should mention that the case of direct hadronization in the absence of a photosphere or chromosphere is already the subject of investigations using PYTHIA 6.2 with the CHARYBDIS event generator, leading again to a hadron dominated spectrum 321 . Other contributions on the numerical side include the TRUENOIR ${ }^{322}$ black hole event generator and, more recently, CATFISH ${ }^{323}$ and BlackMax 324 which employ a richer set of external parameters and allows a certain flexibility among different theoretical models.

In addition to all of this, the detection of stable black hole remnants would provide further interesting new signatures favoring the Noncommutative Geometry inspired models. Electrically charged remnants would leave an ionizing track in the detector, allowing their identification and direct measurement of their masses. On the other hand, neutral remnants would not be visible in the detector but could be identified by modifications of the $p_{T}$ distribution, multiplicities and angular correlations of Standard Model particles emitted as Hawking evaporation. The remnants would also carry a fraction of the total energy and therefore a search for events with $\sim \mathrm{TeV}$ missing energy could give a signature for the presence of a minimum length related to $\sqrt{\theta}$. It is generally believed that the large acceptance of the detectors at the LHC will enable a complete event reconstruction to be made so that the missing energy can be determined. The effects of the formation of these remnants on black hole evaporation have been investigated in Refs. 325, 326.

A lot of work is still needed in order to get a definitive picture of the phenomenology of black hole production at the LHC. Even if we have found encouraging cross sections $\sim 20-400 \mathrm{pb}$, a still open problem, at least within the framework of the models presented, regards the threshold energy required to prime the production, since for $M_{\theta} \sim M_{*} \sim 1 \mathrm{TeV}$ and for $d \sim 10$ this could be around $10^{4} \mathrm{TeV}$. The difficulty caused by this could be overcome in the case of black hole events in the Earth's atmosphere originated by ultra high energy cosmic rays 327 for which a scale of around $\sim \mathrm{PeV}$ or even beyond is considered reasonable. Therefore it is very likely that in the near future we could be able to obtain some signatures of the production of tiny black holes in particle collisions, so as to address long standing questions about the quantum nature of gravity. Given the strong hypothesis about the presence of additional dimensions in order to have an experimentally testable scale to work with, any upcoming observations with particle accelerators, at the Pierre Auger Observatory $328 / 329$ or with other experiments, can really be considered as being both the first and the final appeal to Quantum Gravity.

\section{Acknowledgments}

I would like to warmly thanks E. Spallucci for a long-time and successful collaboration on the majority of the topics included in this review. I am also grateful to J. C. Miller for illuminating discussions and careful reading of the draft and A. Smailagic for the collaboration on this area. I would like to express my gratitude also to S. Ansoldi, R. Balbinot, R. Casadio, M. Cobal, M. Cavaglià, G. Giannini, 
A. Gruppuso, S. D. H. Hsu, D. A. Singleton, M. Tessarotto for fruitful discussions and/or comments and references. Finally I want to thank E. T. Akhmedov, H. Balasin, S. Carlip, V. Cardoso, B. Harms, R. Jackiw, D. Grumiller, H. Nicolai, A. C. Ottewill, W. Pezzaglia and T. G. Rizzo for all their support, interest and valuable hints since the early stages of this developing research area.

This work has been partially supported by a CSU Fresno International activity grant.

\section{Appendix A. Mathematical Formulas}

Definitions of incomplete lower $\gamma$ and upper $\Gamma$ functions

$$
\begin{aligned}
\gamma\left(\frac{m}{2}+1, x^{2}\right) & \equiv \int_{0}^{x^{2}} d t t^{m / 2} e^{-t} \\
\Gamma\left(\frac{m}{2}+1, x^{2}\right) & \equiv \int_{x^{2}}^{\infty} d t t^{m / 2} e^{-t}=\Gamma\left(\frac{m}{2}+1\right)-\gamma\left(\frac{m}{2}+1, x^{2}\right) .
\end{aligned}
$$

Integral and differential properties of incomplete $\gamma$ function

$$
\begin{aligned}
& \int_{0}^{r} \frac{d x}{x^{m+1}} \gamma\left(\frac{m}{2}+1, x^{2}\right)=-\frac{1}{2} \frac{\gamma\left(\frac{m}{2}, r^{2}\right)}{r^{m}} \\
& \oint_{\text {sphere }} d^{m} x \rho_{\theta}\left(x^{2}\right)=M \frac{\gamma\left(\frac{m}{2}, R^{2}\right)}{\Gamma\left(\frac{m}{2}\right)} \\
& \gamma^{\prime}\left(\frac{m}{2}, x^{2}\right)=2 x^{m-1} e^{-x^{2}} \\
& \gamma\left(\frac{m}{2}+1, \frac{r^{2}}{4 \theta}\right)=\frac{m}{2} \gamma\left(\frac{m}{2}, \frac{r^{2}}{4 \theta}\right)-\left(\frac{r}{2 \sqrt{\theta}}\right)^{m} e^{-r^{2} / 4 \theta} .
\end{aligned}
$$

Long and short distance behavior of incomplete $\gamma$ functions

$$
\begin{aligned}
& \left.\gamma\left(\frac{m}{2}, x^{2}\right)\right|_{x>>1}=\frac{2}{m} x^{m} e^{-x^{2}}\left[1-\frac{2}{m+2} x^{2}+\frac{2}{m+2} \frac{2}{m+4} x^{4}+\ldots\right] \\
& \left.\gamma\left(\frac{3}{2}, \frac{r^{2}}{4 \theta}\right)\right|_{\frac{r^{2}}{4 \theta}<<1} \approx \frac{r^{3}}{12 \sqrt{\theta^{3}}}\left(1-\frac{7}{20} \frac{r^{2}}{\theta}\right) \\
& \left.\Gamma\left(\frac{m}{2}, x^{2}\right)\right|_{x>>1}=x^{m-2} e^{-x^{2}}\left[1+\left(\frac{m}{2}-1\right) \frac{1}{x^{2}}+\left(\frac{m}{2}-1\right)\left(\frac{m}{2}-2\right) \frac{1}{x^{4}}+\ldots\right] \\
& \left.\gamma\left(\frac{3}{2}, \frac{r^{2}}{4 \theta}\right)\right|_{\frac{r^{2}}{4 \theta}>>1}=\frac{\sqrt{\pi}}{2}-\left.\Gamma\left(\frac{3}{2} \frac{r^{2}}{4 \theta}\right)\right|_{\frac{r^{2}}{4 \theta}>>1} \approx \frac{\sqrt{\pi}}{2}+\frac{1}{2} \frac{r}{\sqrt{\theta}} e^{-\frac{r^{2}}{4 \theta}} .
\end{aligned}
$$

\section{References}

1. S. W. Hawking, Commun. Math. Phys. 43, 199 (1975) [Erratum-ibid. 46, 206 (1976)].

2. B. S. DeWitt, Quantum Field Theory In Curved Space, AIP Conf. Proc. 23, 660 (1975).

3. B. S. DeWitt, Phys. Rept. 19, 295 (1975). 
4. N. D. Birrell and P. C. W. Davies, Quantum Fields In Curved Space, (Cambridge, UK: Univ. Pr. - Cambridge Monographs On Mathematical Physics, 1982)

5. M. K. Parikh and F. Wilczek, Phys. Rev. Lett. 85, 5042 (2000).

6. M. K. Parikh, Int. J. Mod. Phys. D 13, 2351 (2004) [Gen. Rel. Grav. 36, 2419 (2004)].

7. K. Srinivasan and T. Padmanabhan, Phys. Rev. D 60, 024007 (1999).

8. S. Shankaranarayanan, T. Padmanabhan and K. Srinivasan, Class. Quant. Grav. 19, 2671 (2002).

9. E. C. Vagenas, Phys. Lett. B 559, 65 (2003).

10. A. J. M. Medved and E. C. Vagenas, Mod. Phys. Lett. A 20, 1723 (2005).

11. A. J. M. Medved and E. C. Vagenas, Mod. Phys. Lett. A 20, 2449 (2005).

12. M. Arzano, A. J. M. Medved and E. C. Vagenas, JHEP 0509, 037 (2005).

13. M. Angheben, M. Nadalini, L. Vanzo and S. Zerbini, JHEP 0505, 014 (2005).

14. M. Nadalini, L. Vanzo and S. Zerbini, J. Phys. A 39, 6601 (2006).

15. R. Kerner and R. B. Mann, Phys. Rev. D 73, 104010 (2006).

16. B. D. Chowdhury, Pramana 70, 593 (2008).

17. T. K. Nakamura, arXiv:0706.2916 [hep-th].

18. R. Banerjee and B. R. Majhi, Phys. Lett. B 662, 62 (2008).

19. R. Banerjee and B. R. Majhi, JHEP 0806, 095 (2008).

20. E. T. Akhmedov, V. Akhmedova and D. Singleton, Phys. Lett. B 642, 124 (2006).

21. E. T. Akhmedov, V. Akhmedova, T. Pilling and D. Singleton, Int. J. Mod. Phys. A 22, 1705 (2007).

22. E. T. Akhmedov, T. Pilling and D. Singleton, Subtleties in the quasi-classical calculation of Hawking radiation, arXiv:0805.2653 [gr-qc].

23. M. B. Green, J. H. Schwarz and E. Witten, Superstring theory. Vol. 1: Introduction, (Cambridge, UK: Univ. Pr. - Cambridge Monographs On Mathematical Physics, 1987)

24. M. B. Green, J. H. Schwarz and E. Witten, Superstring Theory. Vol. 2: Loop Amplitudes, Anomalies And Phenomenology, (Cambridge, UK: Univ. Pr. - Cambridge Monographs On Mathematical Physics, 1987)

25. C. Rovelli, Quantum Gravity, (Cambridge, UK: Univ. Pr.- Cambridge Monographs On Mathematical Physics, 2004)

26. M. Pavšič, The Landscape of Theoretical Physics: A Global View: From Point Particles to the Brane World and Beyond, in Search of a Unifying Principle, (Kluwer Academic Publisher, Dordrecht, The Netherlands, 2001).

27. W. M. Pezzaglia, Found. Phys. Lett. 5, 57 (1992)

28. W. M. Pezzaglia, Dimensionally democratic calculus and principles of polydimensional physics, in The proceedings of 5th International Conference on Clifford algebras and their applications in mathematical physics, Ixtapa-Zihuatanejo, Mexico, 27 Jun - 4 Jul 1999, eds. R. Ablamowicz, R. and B. Fauser (Birkhauser, 2000) vol. 1, $101-124$.

29. A. Aurilia, G. Denardo, F. Legovini and E. Spallucci, Nucl. Phys. B 252, 523 (1985).

30. A. Aurilia, R. S. Kissack, R. Mann and E. Spallucci, Phys. Rev. D 35, 2961 (1987).

31. A. Aurilia, M. Palmer and E. Spallucci, Phys. Rev. D 40, 2511 (1989).

32. A. Aurilia and E. Spallucci, Phys. Lett. B 251, 39 (1990).

33. A. Aurilia, R. Balbinot and E. Spallucci, Phys. Lett. B 262, 222 (1991).

34. S. Ansoldi, A. Aurilia, R. Balbinot and E. Spallucci, Class. Quant. Grav. 14, 2727 (1997).

35. J. H. Taylor and J. M. Weisberg, Astrophys. J. 345, 434 (1989).

36. T. Kinoshita, History of original ideas and basic discoveries in particle physics, $\mathrm{H}$. 
Newman and T. Ypsilantis eds. (Plenum Press, New York, 1995).

37. http://lhc.web.cern.ch/lhc/

38. P. C. Argyres, S. Dimopoulos and J. March-Russell, Phys. Lett. B 441, 96 (1998).

39. S. Dimopoulos and G. L. Landsberg, Phys. Rev. Lett. 87, 161602 (2001).

40. S. D. H. Hsu, Phys. Lett. B 555, 92 (2003).

41. A. Casanova and E. Spallucci, Class. Quant. Grav. 23, R45 (2006).

42. J. L. Feng and A. D. Shapere, Phys. Rev. Lett. 88, 021303 (2002).

43. M. Cavaglia, Int. J. Mod. Phys. A 18, 1843 (2003).

44. H. Stoecker, J. Phys. G 32, S429 (2006).

45. R. Casadio and B. Harms, Int. J. Mod. Phys. A 17, 4635 (2002).

46. M. Cavaglia, S. Das and R. Maartens, Class. Quant. Grav. 20, L205 (2003).

47. S. B. Giddings, Black hole production in TeV-scale gravity, and the future of high energy physics, in Proc. of the APS/DPF/DPB Summer Study on the Future of Particle Physics (Snowmass 2001), Snowmass, Colorado, 30 Jun - 21 Jul 2001, pp P328, ed. N. Graf (SLAC, 2001).

48. M. Bleicher, S. Hofmann, S. Hossenfelder and H. Stoecker, Phys. Lett. B 548, 73 (2002).

49. M. Stenmark, Chin. J. Phys. 40 (2002) 512.

50. A. Chamblin, F. Cooper and G. C. Nayak, Phys. Rev. D 70, 075018 (2004).

51. M. Cavaglia and S. Das, Class. Quant. Grav. 21, 4511 (2004).

52. T. G. Rizzo, Class. Quant. Grav. 23, 4263 (2006).

53. H. Falcke, What is SGR A*?: The Starved black hole in the center of the milky way, Invited review to IUA Symposium 'Unsolved Problems of the Milky Way,' Hague, Netherlands, 23-27 Aug 1994. Published in Unsolved Problems of the Milky Way, IAU Symp. 169, L. Blitz and P.J. Teuben (eds.), (Kluwer, Dordrecht, 1996), p. 163-175.

54. R. Schodel et al., Nature 419, 694 (2002).

55. A. Eckart and C. Straubmeier, The supermassive black hole at the center of the Milky Way, in Tegernsee 2003, Beyond the desert, ed. H. V. Klapdor-Kleingrothaus (Springer, Berlin, 2004), p. 627-638.

56. A. M. Ghez et al., Astrophys. J. 601, L159 (2004).

57. D. Sanwal, E. L. Robinson, E. Zhang, C. Colome, P. M. Harvey, T. F. Ramseyer, Astrophys. J. 460, 437 (1996)

58. R. I. Hynes et al., Mon. Not. Roy. Astron. Soc. 330, 1009 (2002).

59. T. Shahbaz et al., Mon. Not. Roy. Astron. Soc. 346, 1116 (2003).

60. C. Zurita, J. Casares, R. I. Hynes, T. Shahbaz, P. A. Charles and E. P. Pavlenko, Mon. Not. Roy. Astron. Soc. 352, 877 (2004).

61. R. I. Hynes et al., Astrophys. J. 611, L125 (2004).

62. S. Corbel, E. Koerding and P. Kaaret, Revisiting the radio/X-ray flux correlation in the black hole V404 Cyg: from outburst to quiescence, arXiv:0806.3079 [astro-ph].

63. D. N. Page and S. W. Hawking, Astrophys. J. 206, 1 (1976).

64. W. G. Unruh, Phys. Rev. Lett. 46, 1351 (1981).

65. R. Balbinot, A. Fabbri, S. Fagnocchi and R. Parentani, Riv. Nuovo Cim. 28, 1 (2005).

66. R. Balbinot, A. Fabbri and S. Fagnocchi, Non-local density correlations as signal of Hawking radiation in BEC acoustic black holes, arXiv:0711.4520 [cond-mat.other].

67. I. Carusotto, S. Fagnocchi, A. Recati, R. Balbinot and A. Fabbri, New J. Phys. 10, 103001 (2008).

68. E. T. Akhmedov and D. Singleton, Pisma Zh. Eksp. Teor. Fiz. 86, 702 (2007).

69. E. T. Akhmedov and D. Singleton, Int. J. Mod. Phys. A 22, 4797 (2007).

70. N. Arkani-Hamed, S. Dimopoulos and G. R. Dvali, Phys. Lett. B 429, 263 (1998). 
71. I. Antoniadis, N. Arkani-Hamed, S. Dimopoulos and G. R. Dvali, Phys. Lett. B 436, 257 (1998).

72. N. Arkani-Hamed, S. Dimopoulos and G. R. Dvali, Phys. Rev. D 59, 086004 (1999).

73. M. Gogberashvili, Mod. Phys. Lett. A 14, 2025 (1999)

74. M. Gogberashvili, Europhys. Lett. 49, 396 (2000).

75. M. Gogberashvili, Int. J. Mod. Phys. D 11, 1635 (2002).

76. M. Gogberashvili, Int. J. Mod. Phys. D 11, 1639 (2002).

77. C. Kokorelis, Nucl. Phys. B 677, 115 (2004).

78. L. Randall and R. Sundrum, Phys. Rev. Lett. 83, 3370 (1999).

79. L. Randall and R. Sundrum, Phys. Rev. Lett. 83, 4690 (1999).

80. C. D. Hoyle, D. J. Kapner, B. R. Heckel, E. G. Adelberger, J. H. Gundlach, U. Schmidt and H. E. Swanson, Phys. Rev. D 70, 042004 (2004).

81. D. J. Kapner, T. S. Cook, E. G. Adelberger, J. H. Gundlach, B. R. Heckel, C. D. Hoyle and H. E. Swanson, Phys. Rev. Lett. 98, 021101 (2007).

82. M. Bleicher, Eur. J. Phys. 28, 509 (2007).

83. A. Strominger and C. Vafa, Phys. Lett. B 379, 99 (1996).

84. C. G. Callan and J. M. Maldacena, Nucl. Phys. B 472, 591 (1996).

85. T. Padmanabhan, Phys. Rept. 406, 49 (2005).

86. B. S. DeWitt, Phys. Rev. 160, 1113 (1967).

87. B. S. DeWitt, Phys. Rev. 162, 1195 (1967).

88. B. S. DeWitt, Phys. Rev. 162, 1239 (1967).

89. B. S. DeWitt, The Formal Structure Of Quantum Gravity, in Cargese 1978, Proceedings, Recent Developments In Gravitation, 275-322 (Springer-Verlag, 1978).

90. L. J. Garay, Int. J. Mod. Phys. A 10, 145 (1995).

91. X. Calmet, M. Graesser and S. D. H. Hsu, Phys. Rev. Lett. 93, 211101 (2004).

92. W. Pauli, Letter of Heisenberg to Peierls (1930), in Scientific Correspondence, ed. Karl von Meyenn (Springer-Verlag, 1985), p. 15, Vol II.

93. E. Schrödinger, Die Naturwiss. 31, 518 (1934).

94. A. Mach, Z. Phys. 104, 93 (1937).

95. W. Heisenberg, Ann. Phys. 32, 20 (1938).

96. H. S. Snyder, Phys. Rev. 71, 38 (1947).

97. C. N. Yang, Phys. Rev. 72, 874 (1947).

98. A. Connes, Inst. Hautes Etudes Sci. Publ. Math. Phys. 22, 257 (1985).

99. S. L. Woronowicz, Publ. Res. Inst. Math. Sci. Kyoto 23, 117 (1987).

100. S. L. Woronowicz, Commun. Math. Phys. 111, 613 (1987).

101. A. Connes, Noncommutative geometry, (Academic Press, 1994).

102. A. Connes, Commun. Math. Phys. 182, 155 (1996).

103. G. Landi, An Introduction to Noncommutative Spaces and their Geometries, (Springer-Verlag, 1997).

104. J. Madore, An Introduction to Noncommutative Geometry and its Physical Applications, (Cambridge University Press, 1999).

105. J. M. Garcia-Bondía, J. C. Várilly and H. Figueroa, Elements of Noncommutative Geometry, (Birkhäuser, 2000).

106. A. Connes, J. Math. Phys. 41, 3832 (2000).

107. E. Witten, Nucl. Phys. B 268, 253 (1986).

108. N. Seiberg and E. Witten, JHEP 9909, 032 (1999).

109. T. Thiemann, Introduction to modern canonical quantum general relativity, arXiv:gr-qc/0110034

110. A. Ashtekar, A. Corichi and J. A. Zapata, Class. Quant. Grav. 15, 2955 (1998).

111. A. Corichi and J. A. Zapata, Int. J. Mod. Phys. D 17, 445 (2008). 
112. A. Ashtekar, Loop quantum gravity: Four recent advances and a dozen frequently asked questions, to appear in Proceedings of 11th Marcel Grossmann Meeting on Recent Developments in Theoretical and Experimental General Relativity, Gravitation, and Relativistic Field Theories, Berlin, Germany, 23-29 Jul 2006, edited by H. Kleinert, R.T. Jantzen and R. Ruffini (World Scientific, Singapore, 2008) arXiv:0705.2222 [gr-qc].

113. M. R. Douglas and N. A. Nekrasov, Rev. Mod. Phys. 73, 977 (2001).

114. R. J. Szabo, Phys. Rept. 378, 207 (2003).

115. S. Doplicher, K. Fredenhagen and J. E. Roberts, Phys. Lett. B 331, 39 (1994).

116. S. Doplicher, K. Fredenhagen and J. E. Roberts, Commun. Math. Phys. 172, 187 (1995).

117. K. Morita, Prog. Theor. Phys. 108, 1099 (2003).

118. H. Kase, K. Morita, Y. Okumura and E. Umezawa, Prog. Theor. Phys. 109, 663 (2003).

119. K. Morita, Y. Okumura and E. Umezawa, Prog. Theor. Phys. 110, 989 (2003).

120. K. Morita, Prog. Theor. Phys. 111, 881 (2004).

121. A. Smailagic and E. Spallucci, J. Phys. A 37, 1 (2004) [Erratum-ibid. A 37, 7169 (2004)].

122. H. J. Groenewold, Physica 12, 405 (1946).

123. J. E. Moyal, Proc. Cambridge Phil. Soc. 45, 99 (1949).

124. H. Weyl, The Theory of Groups and Quantum Mechanics, (Dover, New York, 1931).

125. E. P. Wigner, Phys. Rev. 40, 749 (1932).

126. D. Bahns, S. Doplicher, K. Fredenhagen and G. Piacitelli, Phys. Lett. B 533, 178 (2002).

127. D. Bahns, S. Doplicher, K. Fredenhagen and G. Piacitelli, Commun. Math. Phys. 237, 221 (2003)

128. H. Grosse and R. Wulkenhaar, Commun. Math. Phys. 256, 305 (2005).

129. R. J. Glauber, Phys. Rev. 131, 2766 (1963).

130. S. Cho, R. Hinterding, J. Madore and H. Steinacker, Int. J. Mod. Phys. D 9, 161 (2000).

131. A. Smailagic and E. Spallucci, J. Phys. A 36, L467 (2003).

132. A. Smailagic and E. Spallucci, J. Phys. A 36, L517 (2003).

133. A. H. Chamseddine, G. Felder and J. Frohlich, Commun. Math. Phys. 155, 205 (1993).

134. J. Madore and J. Mourad, Int. J. Mod. Phys. D 3, 221 (1994).

135. A. Jevicki and S. Ramgoolam, JHEP 9904, 032 (1999).

136. N. E. Mavromatos and R. J. Szabo, Phys. Rev. D 59, 104018 (1999).

137. J. W. Moffat, Phys. Lett. B 491, 345 (2000).

138. A. H. Chamseddine, Phys. Lett. B 504, 33 (2001).

139. A. H. Chamseddine, Commun. Math. Phys. 218, 283 (2001).

140. H. Nishino and S. Rajpoot, Phys. Lett. B 532, 334 (2002).

141. M. A. Cardella and D. Zanon, Class. Quant. Grav. 20, L95 (2003).

142. H. Garcia-Compean, O. Obregon, C. Ramirez and M. Sabido, Phys. Rev. D 68, 044015 (2003).

143. D. V. Vassilevich, Nucl. Phys. B 715, 695 (2005).

144. P. Aschieri, C. Blohmann, M. Dimitrijevic, F. Meyer, P. Schupp and J. Wess, Class. Quant. Grav. 22, 3511 (2005).

145. X. Calmet and A. Kobakhidze, Phys. Rev. D 72, 045010 (2005).

146. D. Bahns, S. Doplicher, K. Fredenhagen and G. Piacitelli, Phys. Rev. D 71, 025022 (2005). 
147. L. Alvarez-Gaume, F. Meyer and M. A. Vazquez-Mozo, Nucl. Phys. B 753, 92 (2006).

148. P. Aschieri, M. Dimitrijevic, F. Meyer and J. Wess, Class. Quant. Grav. 23, 1883 (2006).

149. P. Mukherjee and A. Saha, Phys. Rev. D 74, 027702 (2006).

150. X. Calmet and A. Kobakhidze, Phys. Rev. D 74, 047702 (2006).

151. M. Chaichian, A. Tureanu and G. Zet, Phys. Lett. B 660, 573 (2008).

152. M. Kontsevich, Lett. Math. Phys. 66, 157 (2003).

153. E. Harikumar and V. O. Rivelles, Class. Quant. Grav. 23, 7551 (2006).

154. A. Kobakhidze, Noncommutative corrections to classical black holes, arXiv:0712.0642 [gr-qc].

155. H. Steinacker, JHEP 0712, 049 (2007).

156. F. Nasseri, Gen. Rel. Grav. 37, 2223 (2005).

157. F. Nasseri, Int. J. Mod. Phys. D 15, 1113 (2006).

158. A. Gruppuso, J. Phys. A 38 (2005) 2039.

159. P. Nicolini, A. Smailagic and E. Spallucci, The fate of radiating black holes in noncommutative geometry, in Proc. of EPS-13, "Beyond Einstein - Physics for 21st Century", eds. A. M. Cruise and L. Ouwehand (ESA Publication Division, Noordwijk, The Netherlands, 2006), p. ???.

160. P. Nicolini, Noncommutative nonsingular black holes, in Bled workshops in physics, Vol.6, No. 2 - Proceedings to the 8th Workshop "What Comes Beyond the Standard Models", Bled, July 19. - 29., 2005, Slovenia, eds. N. Mankoc Borstnik, H. Bech Nielsen, C.D. Froggatt, D. Lukman (DMFA-Zaloznistvo, Ljubljana, Slovenia, 2005), p. 79 .

161. P. Nicolini, J. Phys. A 38, L631 (2005).

162. P. Nicolini, A. Smailagic and E. Spallucci, Phys. Lett. B 632, 547 (2006).

163. H. Balasin and H. Nachbagauer, Class. Quant. Grav. 10, 2271 (1993).

164. A. DeBenedictis, Developments in Black Hole Research: Classical, Semi-classical, and Quantum, arXiv:0711.2279 [gr-qc].

165. Y. B. Zel'dovich, Sov. Phys. Usp. 11, 381 (1968).

166. A. Aurilia, G. Denardo, F. Legovini and E. Spallucci, Phys. Lett. B 147, 258 (1984).

167. V. P. Frolov, M. A. Markov and V. F. Mukhanov, Phys. Lett. B 216, 272 (1989)

168. V. P. Frolov, M. A. Markov and V. F. Mukhanov, Phys. Rev. D 41, 383 (1990).

169. S. Ansoldi, Spherical black holes with regular center: a review of existing models including a recent realization with Gaussian sources, to appear in Proceedings of Conference on Black Holes and Naked Singularities, Milan, Italy, 10-12 May 2007, arXiv:0802.0330 [gr-qc].

170. P. S. Letelier, Phys. Rev. D 20, 1294 (1979).

171. I. Dymnikova, Gen. Rel. Grav. 24, 235 (1992).

172. M. Visser, Phys. Rev. D 46, 2445 (1992).

173. K. A. Bronnikov, Phys. Rev. D 63, 044005 (2001).

174. I. Dymnikova, Int. J. Mod. Phys. D 12, 1015 (2003).

175. H. Hernandez and L. A. Nunez, Can. J. Phys. 82, 29 (2004).

176. H. Abreu, H. Hernandez and L. A. Nunez, Class. Quant. Grav. 24, 4631 (2007).

177. K. A. Bronnikov and O. B. Zaslavskii, Black holes can have curly hair, arXiv:0801.0889 [gr-qc].

178. J. M. McNamara, Proc. R. Soc. London A 358, 499 (1978).

179. J. M. McNamara, Proc. R. Soc. London A 364, 121 (1978).

180. Y. Gürsel, V. D. Sandberg, I. D. Novikov and A. A. Starobinsky, Phys. Rev. D 19, 413 (1979).

181. Y. Gürsel, I. D. Novikov, V. D. Sandberg and A. A. Starobinsky, Phys. Rev. D 20, 
1260 (1979).

182. R. A. Matzner, N. Zamorano, and V. D. Sandberg, Phys. Rev. D 19, 2821 (1979).

183. S. Chandrasekhar and J. B. Hartle, Proc. Roy. Soc. Lond. A 384, 301 (1982).

184. E. Poisson and W. Israel, Phys. Rev. D 41, 1796 (1990).

185. L. M. Burko and A. Ori, Phys. Rev. Lett. 74, 1064 (1995).

186. D. Markovic and E. Poisson, Phys. Rev. Lett. 74, 1280 (1995).

187. D. M. Eardley, Phys. Rev. Lett. 33, 442 (1974).

188. V. P. Frolov, Zh. Eksp. Teor. Fiz. 66, 813 (1974).

189. D. M. Eardley and W. H. Press, Ann. Rev. Astron. Astrophys. 13, 381 (1975).

190. I. Redmount, Prog. of Theor. Phys. 73, 1401 (1985).

191. P. O. Mazur and E. Mottola, Gravitational condensate stars, arXiv:gr-qc/0109035

192. S. W. Hawking, Phys. Rev. D 72, 084013 (2005).

193. E. Di Grezia, G. Esposito and G. Miele, Class. Quant. Grav. 23, 6425 (2006).

194. K. Nozari and B. Fazlpour, Mod. Phys. Lett. A 22, 2917 (2007).

195. Y. S. Myung, Y. W. Kim and Y. J. Park, JHEP 0702, 012 (2007).

196. Y. S. Myung, Y. W. Kim and Y. J. Park, Phys. Lett. B 656, 221 (2007).

197. Y. S. Myung, Y. W. Kim and Y. J. Park, Phys. Lett. B 659, 832 (2008).

198. Y. S. Myung, Y. W. Kim and Y. J. Park, Thermodynamics of regular black hole, arXiv:0708.3145 [gr-qc].

199. R. Banerjee, B. R. Majhi and S. Samanta, Phys. Rev. D 77, 124035 (2008).

200. E. Spallucci, A. Smailagic and P. Nicolini, Phys. Lett. B 670, 449 (2009).

201. W. Kim, E. J. Son and M. Yoon, JHEP 0804, 042 (2008).

202. R. Banerjee, B. R. Majhi and S. K. Modak, Area Law in Noncommutative Schwarzschild Black Hole, arXiv:0802.2176 [hep-th].

203. S. Weinberg, in General Relativity: an Einstein Centenary survey, S. W. Hawking and W. Israel Editors, Cambridge University Press, 1979.

204. A. Bonanno and M. Reuter, Phys. Rev. D 62, 043008 (2000).

205. D. Pavon, Phys. Rev. D 43, 2495 (1991).

206. Y. S. Myung, Mod. Phys. Lett. A 23, 667 (2008).

207. C. F. E. Holzhey and F. Wilczek, Nucl. Phys. B 380, 447 (1992).

208. M. A. Markov, Suppl. Progr. Theor. Phys., 85 (1965).

209. M. A. Markov, Zh. Exsp. Teor. Fiz. 51, 879 (1966).

210. S. W. Hawking, Commun. Math. Phys. 25, 152 (1972).

211. S. Hossenfelder, What black holes can teach us, in Focus on Black Hole Research, ed. Paul V. Kreitler, (Nova Science Publishers, New York, 2005), pp. 155-192, arXiv:hep-ph/0412265.

212. M. A. Markov, Maximon Type Scenario Of The Universe (Big Bang, Small Bang, Microbang), Preprint P-0208 INR, Moscow, 1981.

213. I. D. Novikov and V. P. Frolov, Physics of Black Holes, Fundamental theories of physics, 27 (Dordrecht, Netherlands: Kluwer Academic,1989).

214. J. D. Barrow, E. J. Copeland and A. R. Liddle, Phys. Rev. D 46, 645 (1992).

215. M. J. Bowick, S. B. Giddings, J. A. Harvey, G. T. Horowitz and A. Strominger, Phys. Rev. Lett. 61, 2823 (1988).

216. B. Whitt, Phys. Rev. D 38, 3000 (1988).

217. C. G. . Callan, R. C. Myers and M. J. Perry, Nucl. Phys. B 311, 673 (1989).

218. S. R. Coleman, J. Preskill and F. Wilczek, Mod. Phys. Lett. A 6, 1631 (1991).

219. T. Banks, M. O'Loughlin and A. Strominger, Phys. Rev. D 47, 4476 (1993).

220. D. A. Lowe and M. O'Loughlin, Phys. Rev. D 48, 3735 (1993).

221. J. D. Bekenstein, Phys. Rev. D 49, 1912 (1994).

222. R. J. Adler, P. Chen and D. I. Santiago, Gen. Rel. Grav. 33, 2101 (2001). 
223. S. Alexeyev, A. Barrau, G. Boudoul, O. Khovanskaya and M. Sazhin, Class. Quant. Grav. 19, 4431 (2002).

224. B. Koch, M. Bleicher and S. Hossenfelder, JHEP 0510, 053 (2005).

225. E. Spallucci, A. Smailagic and P. Nicolini, Phys. Rev. D 73, 084004 (2006).

226. O. B. Zaslavskii, Class. Quant. Grav. 8 (1991) L141.

227. R. Balbinot and A. Fabbri, Phys. Rev. D 59, 044031 (1999).

228. D. Grumiller, W. Kummer and D. V. Vassilevich, Phys. Rept. 369, 327 (2002).

229. R. Balbinot, A. Fabbri, V. P. Frolov, P. Nicolini, P. Sutton and A. Zelnikov, Phys. Rev. D 63, 084029 (2001).

230. H. Garcia-Compean and C. Soto-Campos, Phys. Rev. D 74, 104028 (2006).

231. G. A. Vilkovisky, Phys. Lett. B 638 (2006) 523.

232. D. Grumiller and R. Meyer, Turk. J. Phys. 30 (2006) 349.

233. D. Grumiller and R. McNees, JHEP 0704 (2007) 074.

234. K. Nozari and S. Akhshabi, Europhys. Lett. 80, 20002 (2007).

235. P. Mukherjee and A. Saha, Phys. Rev. D 77, 064014 (2008).

236. M. Chaichian, M. R. Setare, A. Tureanu and G. Zet, JHEP 0804, 064 (2008).

237. F. Nasseri and S. A. Alavi, Int. J. Mod. Phys. D 16, 899 (2007).

238. S. Ansoldi, P. Nicolini, A. Smailagic and E. Spallucci, Phys. Lett. B 645, 261 (2007).

239. R. M. Wald, General Relativity, (Chicago, Usa: Univ. Pr., 1984).

240. J. S. Schwinger, Phys. Rev. 82, 664 (1951).

241. A. I. Nikishov, Zh. Eksp. Teor. Fiz. 57, 1210 (1969).

242. M. A. Markov and V. P. Frolov, Teor. Mat. Fiz. 3, 3 (1970).

243. N. Deruelle and R. Ruffini, Phys. Lett. 52B, 437 (1974).

244. G. W. Gibbons, Commun. Math. Phys. 44, 245 (1975).

245. T. Damour and R. Ruffini, Phys. Rev. Lett. 35, 463 (1975).

246. J. K. Daugherty and I. Lerche, Astrophys. Space Sci. 38, 437 (1975).

247. J. K. Daugherty and I. Lerche, Phys. Rev. D 14, 340 (1976).

248. T. Damour and R. Ruffini, Phys. Rev. D 14, 332 (1976).

249. D. Garfinkle, S. B. Giddings and A. Strominger, Phys. Rev. D 49, 958 (1994).

250. R. Parentani and S. Massar, Phys. Rev. D 55, 3603 (1997).

251. I. M. Ternov, A. B. Gaina and G. A. Chizhov, Sov. J. Nucl. Phys. 44, 343 (1986) [Yad. Fiz. 44, 533 (1986)].

252. G. Preparata, R. Ruffini and S. S. Xue, Astron. Astrophys. 338, L87 (1998).

253. M. H. P. van Putten, Phys. Rev. Lett. 84, 3752 (2000).

254. G. Preparata, R. Ruffini and S. S. Xue, J. Korean Phys. Soc. 42, S99 (2003).

255. D. N. Page, Astrophys. J. 653, 1400 (2006).

256. D. N. Page, No Astrophysical Dyadospheres, arXiv:astro-ph/0605432

257. D. N. Page, Dyadospheres Don't Develop, in Proceedings of 7th Asia-Pacific International Conference on Gravitation and Astrophysics (ICGA7 2005), Chungli, Taiwan, 23-26 Nov 2005 (World Scientific, 2007) p. 206-215.

258. J. L. Hewett, F. J. Petriello and T. G. Rizzo, Phys. Rev. D 64, 075012 (2001).

259. H. Grosse and Y. Liao, Phys. Rev. D 64, 115007 (2001).

260. H. Grosse and Y. Liao, Phys. Lett. B 520, 63 (2001).

261. A. Anisimov, T. Banks, M. Dine and M. Graesser, Phys. Rev. D 65, 085032 (2002).

262. J. L. Hewett, F. J. Petriello and T. G. Rizzo, Phys. Rev. D 66, 036001 (2002).

263. C. E. Carlson, C. D. Carone and N. Zobin, Phys. Rev. D 66, 075001 (2002).

264. E. O. Iltan, Phys. Rev. D 66, 034011 (2002).

265. Z. Chang and Z. z. Xing, Phys. Rev. D 66, 056009 (2002).

266. N. G. Deshpande and X. G. He, Phys. Lett. B 533, 116 (2002).

267. C. E. Carlson and C. D. Carone, Phys. Rev. D 65, 075007 (2002). 
268. S. Godfrey and M. A. Doncheski, Phys. Rev. D 65, 015005 (2002).

269. G. Duplancic, P. Schupp and J. Trampetic, Eur. Phys. J. C 32, 141 (2003).

270. J. M. Conroy, H. J. Kwee and V. Nazaryan, Phys. Rev. D 68, 054004 (2003).

271. N. Mahajan, Phys. Rev. D 68, 095001 (2003).

272. X. G. He, Eur. Phys. J. C 28, 557 (2003).

273. W. Behr, N. G. Deshpande, G. Duplancic, P. Schupp, J. Trampetic and J. Wess, Eur. Phys. J. C 29, 441 (2003).

274. I. Hinchliffe, N. Kersting and Y. L. Ma, Int. J. Mod. Phys. A 19, 179 (2004).

275. T. Ohl and J. Reuter, Phys. Rev. D 70, 076007 (2004) .

276. P. Schupp, J. Trampetic, J. Wess and G. Raffelt, Eur. Phys. J. C 36, 405 (2004).

277. P. Minkowski, P. Schupp and J. Trampetic, Eur. Phys. J. C 37, 123 (2004).

278. B. Melic, K. Passek-Kumericki, J. Trampetic, P. Schupp and M. Wohlgenannt, Eur. Phys. J. C 42, 499 (2005).

279. X. Calmet, Eur. Phys. J. C 41, 269 (2005).

280. C. D. Carone and H. J. Kwee, Phys. Rev. D 73, 096005 (2006).

281. A. Alboteanu, T. Ohl and R. Ruckl, PoS HEP2005, 322 (2006).

282. A. Alboteanu, T. Ohl and R. Ruckl, Phys. Rev. D 74, 096004 (2006).

283. M. Buric, D. Latas, V. Radovanovic and J. Trampetic, Phys. Rev. D 75, 097701 (2007)

284. J. Trampetic, Fortsch. Phys. 56, 521 (2008).

285. J. i. Kamoshita, Eur. Phys. J. C 52, 451 (2007).

286. G. F. Giudice, R. Rattazzi and J. D. Wells, Nucl. Phys. B 544, 3 (1999).

287. T. Han, J. D. Lykken and R. J. Zhang, Phys. Rev. D 59, 105006 (1999).

288. E. A. Mirabelli, M. Perelstein and M. E. Peskin, Phys. Rev. Lett. 82, 2236 (1999).

289. J. L. Hewett, Phys. Rev. Lett. 82, 4765 (1999).

290. R. Casadio and B. Harms, Phys. Rev. D 64, 024016 (2001).

291. R. Casadio and B. Harms, Phys. Lett. B 487, 209 (2000).

292. J. L. Hewett and M. Spiropulu, Ann. Rev. Nucl. Part. Sci. 52, 397 (2002).

293. S. B. Giddings and S. D. Thomas, Phys. Rev. D 65, 056010 (2002).

294. P. Kanti, Int. J. Mod. Phys. A 19, 4899 (2004).

295. T. G. Rizzo, JHEP 0506, 079 (2005).

296. J. L. Hewett, B. Lillie and T. G. Rizzo, Phys. Rev. Lett. 95, 261603 (2005).

297. D. M. Gingrich, Int. J. Mod. Phys. A 21, 6653 (2006).

298. G. L. Landsberg, J. Phys. G 32, R337 (2006).

299. H. Stoecker, J. Phys. G 32, S429 (2006).

300. G. L. Alberghi, R. Casadio and A. Tronconi, J. Phys. G 34, 767 (2007).

301. M. Cavaglia, R. Godang, L. M. Cremaldi and D. J. Summers, JHEP 0706, 055 (2007).

302. T. J. Humanic, B. Koch and H. Stoecker, Int. J. Mod. Phys. E 16, 841 (2007).

303. B. Koch, M. Bleicher and H. Stoecker, J. Phys. G 34, S535 (2007).

304. H. Stoecker, Int. J. Mod. Phys. D 16, 185 (2007).

305. A. Roy and M. Cavaglia, Phys. Rev. D 77, 064029 (2008).

306. T. G. Rizzo, JHEP 0609, 021 (2006).

307. R. C. Myers and M. J. Perry, Annals Phys. 172, 304 (1986).

308. M. Nagano and A. A. Watson, Rev. Mod. Phys. 72, 689 (2000).

309. T. Appelquist, H. C. Cheng and B. A. Dobrescu, Phys. Rev. D 64, 035002 (2001).

310. J. M. Smillie and B. R. Webber, JHEP 0510, 069 (2005).

311. A. Datta, K. Kong and K. T. Matchev, Phys. Rev. D 72, 096006 (2005) [Erratumibid. D 72, 119901 (2005)].

312. R. N. Mohapatra and A. Perez-Lorenzana, Phys. Rev. D 67, 075015 (2003). 
313. S. K. Rai, Int. J. Mod. Phys. A 23, 823 (2008).

314. H. Stocker, B. Koch and M. Bleicher, Braz. J. Phys. 37, 836 (2007).

315. C. M. Harris and P. Kanti, JHEP 0310, 014 (2003).

316. R. Emparan, G. T. Horowitz and R. C. Myers, Phys. Rev. Lett. 85, 499 (2000).

317. M. Cavaglia, Phys. Lett. B 569, 7 (2003).

318. P. Kanti and J. March-Russell, Phys. Rev. D 66, 024023 (2002).

319. P. Kanti and J. March-Russell, Phys. Rev. D 67, 104019 (2003).

320. A. F. Heckler, Phys. Rev. D 55, 480 (1997).

321. C. M. Harris, P. Richardson and B. R. Webber, JHEP 0308, 033 (2003).

322. S. Dimopoulos and G. L. Landsberg, Black hole production at future colliders, in Proc. of the APS/DPF/DPB Summer Study on the Future of Particle Physics (Snowmass 30 Jun - 21 Jul 2001), ed. N. Graf, (Snowmass, Colorado, 2001), pp. P321.

323. M. Cavaglia, R. Godang, L. Cremaldi and D. Summers, Comput. Phys. Commun. 177, 506 (2007).

324. D. C. Dai, G. Starkman, D. Stojkovic, C. Issever, E. Rizvi and J. Tseng, Phys. Rev. D 77, 076007 (2008).

325. S. Hossenfelder, B. Koch and M. Bleicher, Trapping black hole remnants, arXiv:hep-ph/0507140.

326. R. Casadio and P. Nicolini, JHEP 0811, 072 (2008).

327. E. J. Ahn and M. Cavaglia, Phys. Rev. D 73, 042002 (2006).

328. D. Stojkovic, G. D. Starkman and D. C. Dai, Phys. Rev. Lett. 96, 041303 (2006).

329. http://www.auger.org/ 